This RFC specifies an Internet standards track protocol for the Internet community, and requests discussion and suggestions for improvements. Please refer to the current edition of the "Internet Official Protocol standards" for the standardization state and status of this protocol. Distribution of this memo is unlimited.

Abstract

This document gives an overview and specification of Version 5 of the protocol for the Kerberos network authentication system. Version 4, described elsewhere [1,2], is presently in production use at $\mathrm{MIT}^{\prime} \mathrm{S}$ Project Athena, and at other Internet sites.

Overview

Project Athena, Athena, Athena MUSE, Discuss, Hesiod, Kerberos, Moira, and Zephyr are trademarks of the Massachusetts Institute of Technology (MIT). No commercial use of these trademarks may be made without prior written permission of MIT.

This RFC describes the concepts and model upon which the Kerberos network authentication system is based. It also specifies Version 5 of the Kerberos protocol.

The motivations, goals, assumptions, and rationale behind most design decisions are treated cursorily; for Version 4 they are fully described in the Kerberos portion of the Athena Technical Plan [1]. The protocols are under review, and are not being submitted for consideration as an Internet standard at this time. Comments are encouraged. Requests for addition to an electronic mailing list for discussion of Kerberos, kerberos@MIT.EDU, may be addressed to kerberos-request@MIT.EDU. This mailing list is gatewayed onto the Usenet as the group comp.protocols.kerberos. Requests for further information, including documents and code availability, may be sent to info-kerberos@MIT. EDU. 


\section{Background}

The Kerberos model is based in part on Needham and Schroeder's trusted third-party authentication protocol [3] and on modifications suggested by Denning and Sacco [4]. The original design and implementation of Kerberos Versions 1 through 4 was the work of two former Project Athena staff members, Steve Miller of Digital Equipment Corporation and Clifford Neuman (now at the Information Sciences Institute of the University of Southern California), along with Jerome Saltzer, Technical Director of Project Athena, and Jeffrey Schiller, MIT Campus Network Manager. Many other members of Project Athena have also contributed to the work on Kerberos. Version 4 is publicly available, and has seen wide use across the Internet.

Version 5 (described in this document) has evolved from Version 4 based on new requirements and desires for features not available in Version 4. Details on the differences between Kerberos Versions 4 and 5 can be found in [5].

Table of Contents

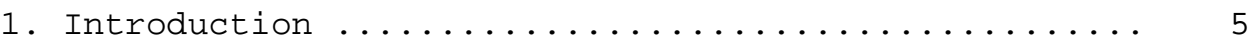

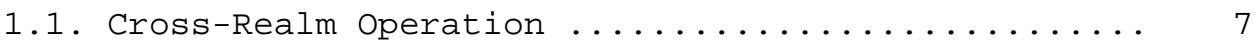

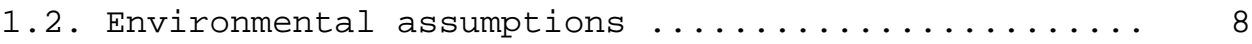

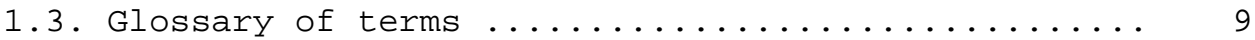

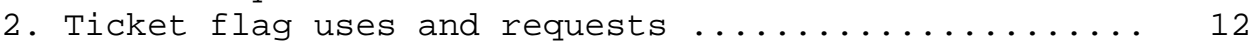

2.1. Initial and pre-authenticated tickets ......... 12

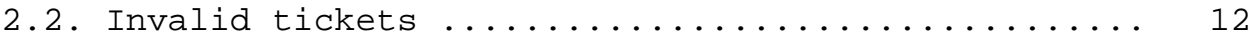

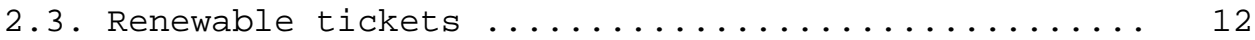

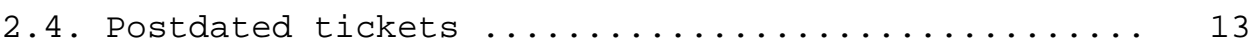

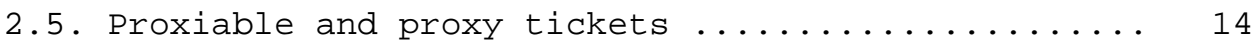

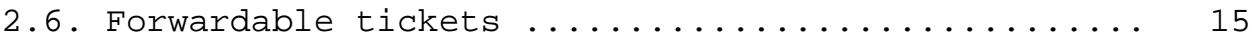

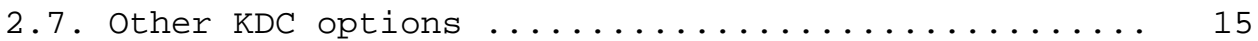

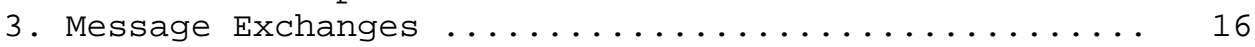

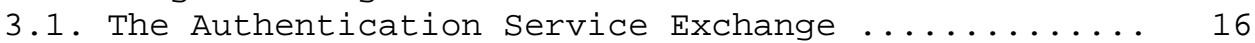

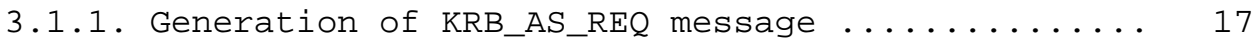

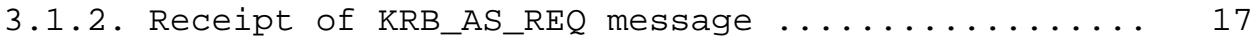

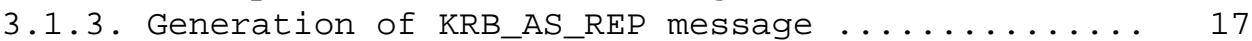

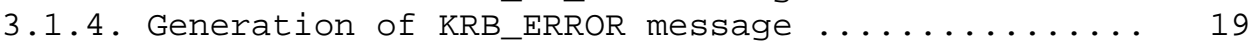

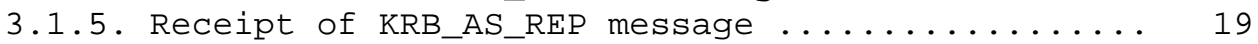

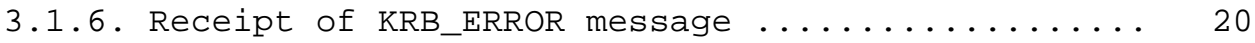

3.2. The Client/Server Authentication Exchange ....... 20

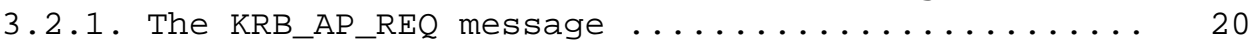

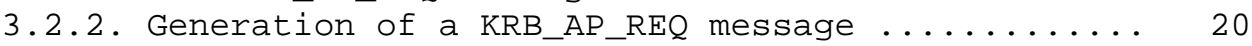

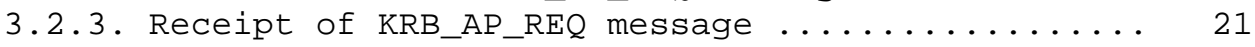

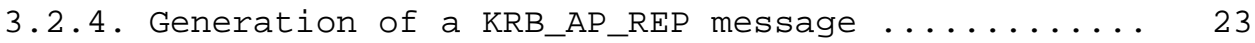

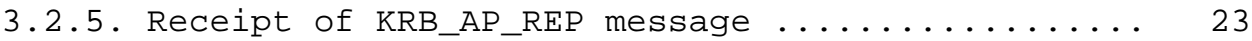




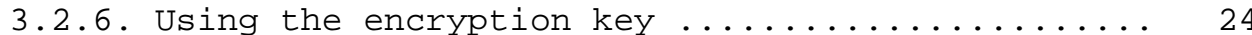

3.3. The Ticket-Granting Service (TGS) Exchange ...... 24

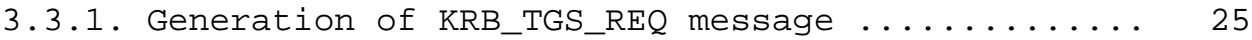

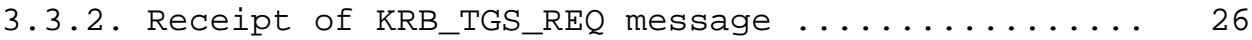

3.3.3. Generation of KRB_TGS_REP message ......... 27

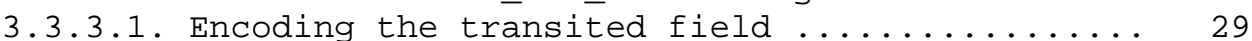

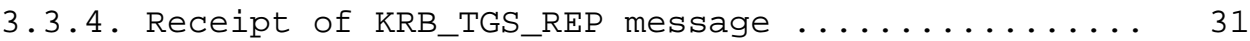

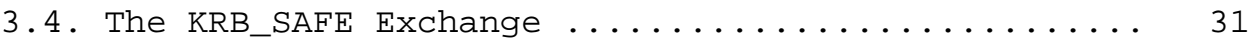

3.4.1. Generation of a KRB_SAFE message ........... 31

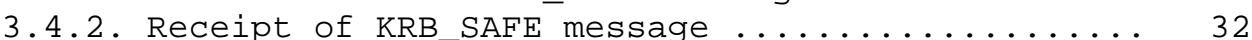

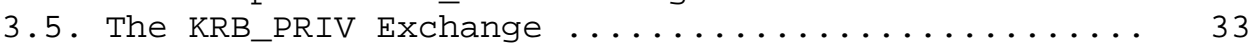

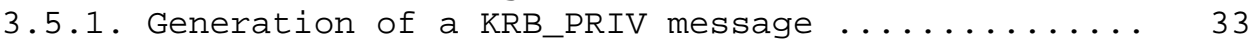

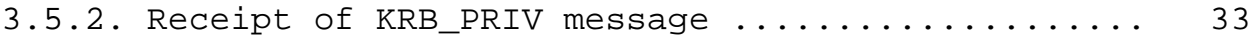

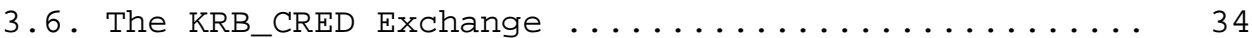

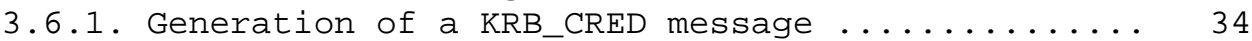

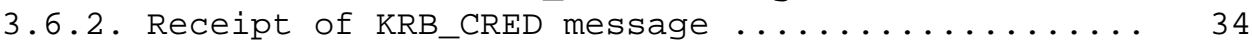

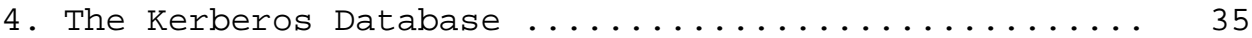

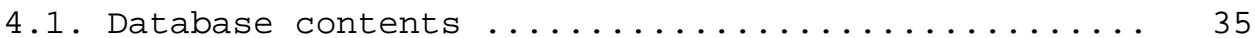

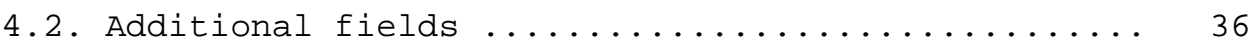

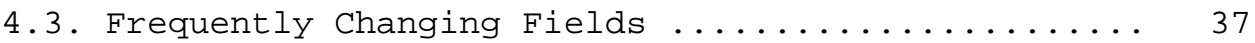

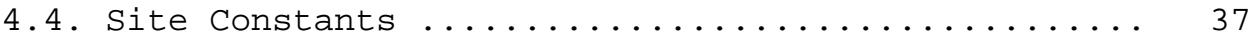

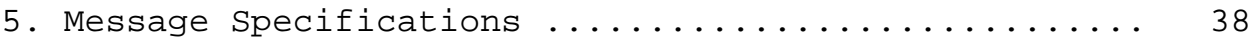

5.1. ASN.1 Distinguished Encoding Representation ..... 38

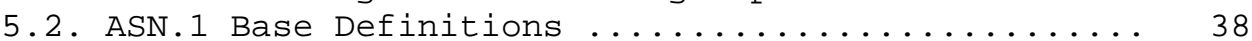

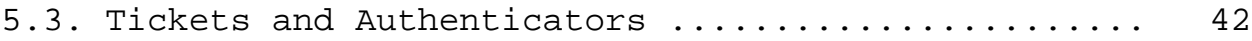

5.3 .1$. Tickets ...................... 42

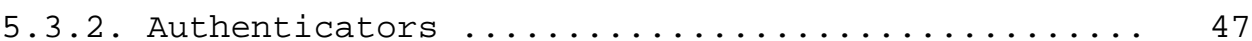

5.4. Specifications for the AS and TGS exchanges ..... 49

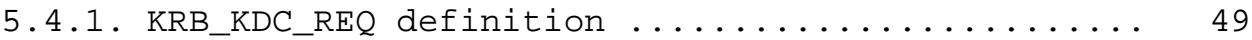

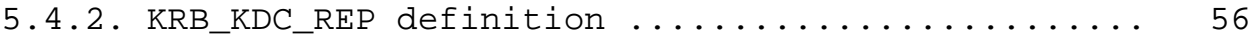

5.5. Client/Server (CS) message specifications ...... 58

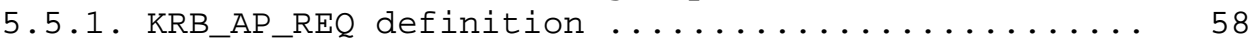

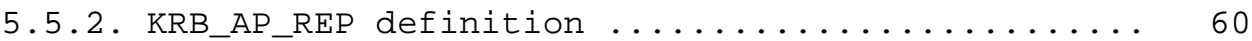

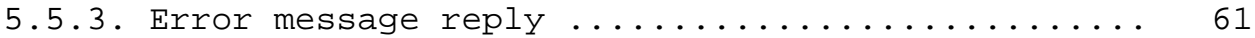

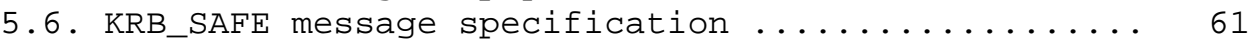

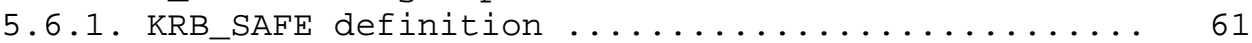

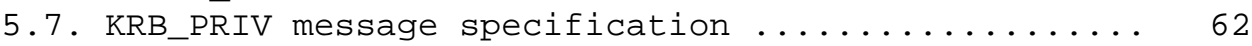

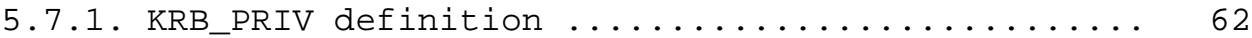

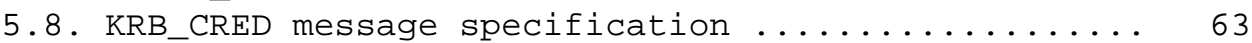

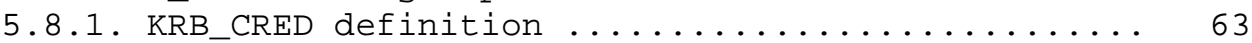

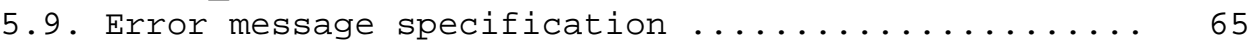

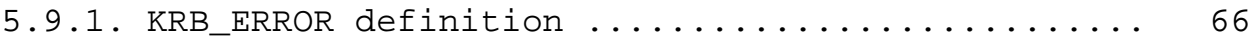

6. Encryption and Checksum Specifications ...................... 67

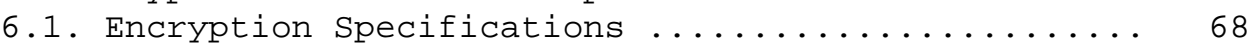

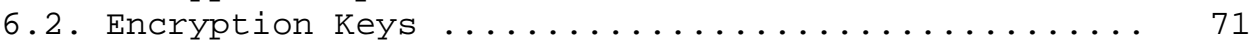

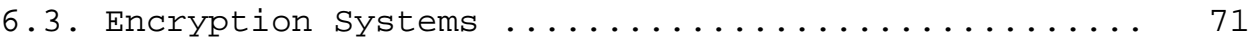

6.3.1. The NULL Encryption System (null) ........... 71

6.3.2. DES in CBC mode with a CRC-32 checksum (descbc-crc) 71 
6.3.3. DES in CBC mode with an MD4 checksum (descbc-md4) 72 6.3.4. DES in CBC mode with an MD5 checksum (descbc-md5) 72

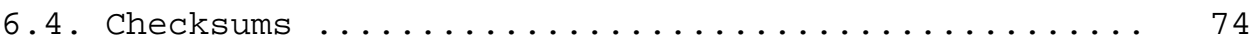

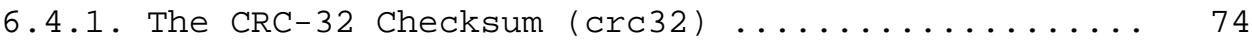
6.4.2. The RSA MD4 Checksum (rsa-md4) ........... 75 6.4.3. RSA MD4 Cryptographic Checksum Using DES

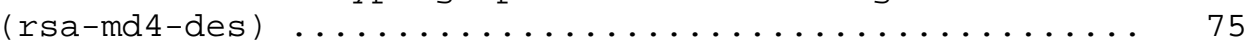
6.4.4. The RSA MD5 Checksum (rsa-md5) ........... 76 6.4.5. RSA MD5 Cryptographic Checksum Using DES

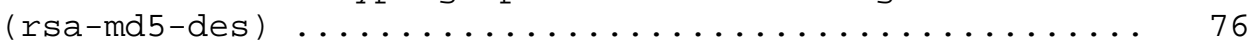
6.4.6. DES cipher-block chained checksum (des-mac) 6.4.7. RSA MD4 Cryptographic Checksum Using DES

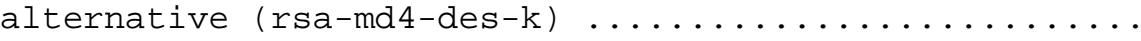
6.4.8. DES cipher-block chained checksum alternative

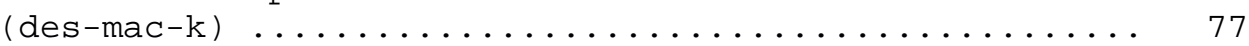

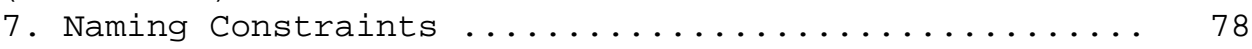

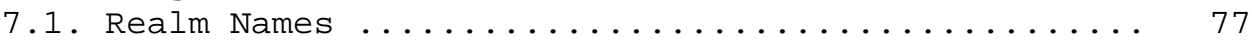

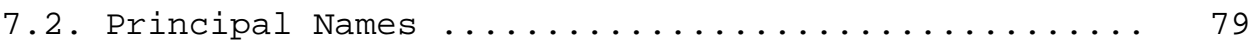

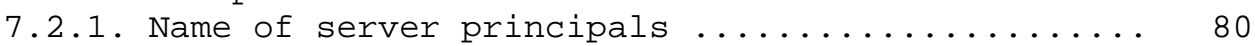

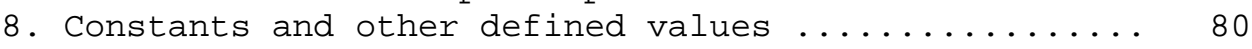

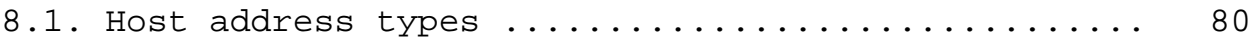

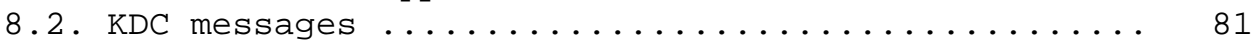

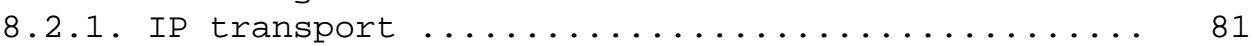

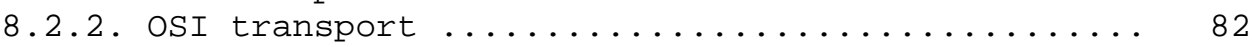

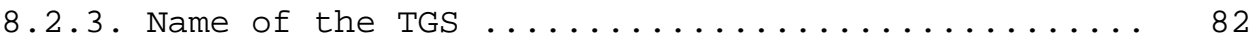

8.3. Protocol constants and associated values ....... 82

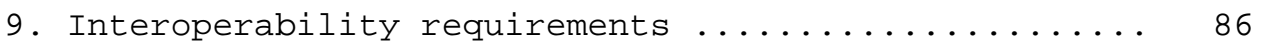

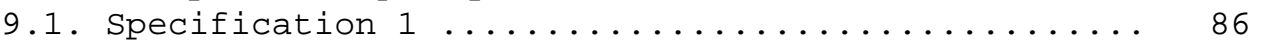

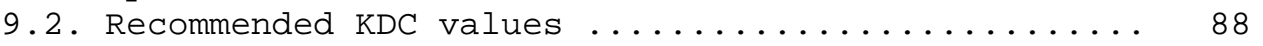

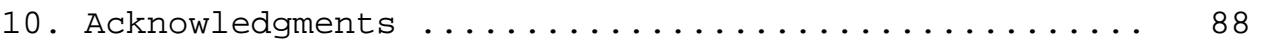

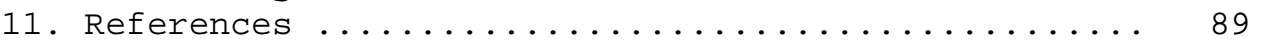

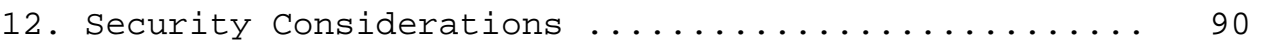

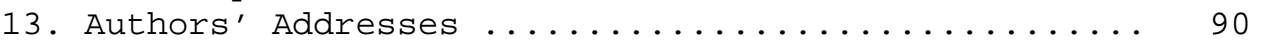

A. Pseudo-code for protocol processing ............ 91

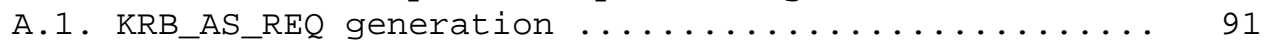

A.2. KRB_AS_REQ verification and KRB_AS_REP generation 92

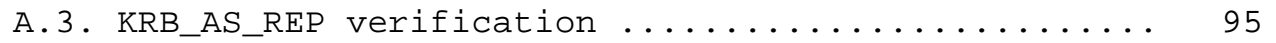

A.4. KRB_AS_REP and KRB_TGS_REP common checks ....... 96

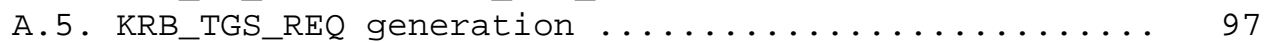

A.6. KRB_TGS_REQ verification and KRB_TGS_REP generation 98

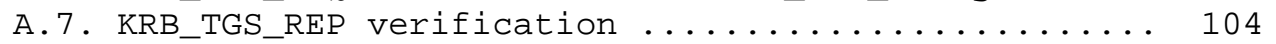

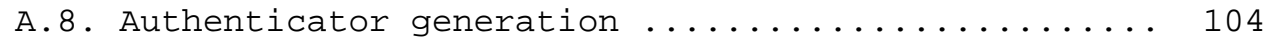

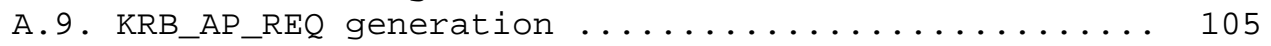

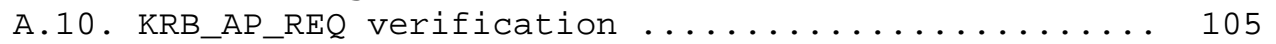

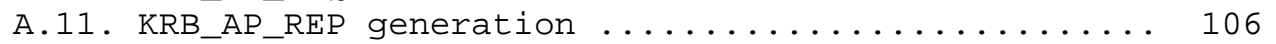

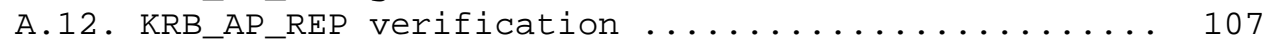

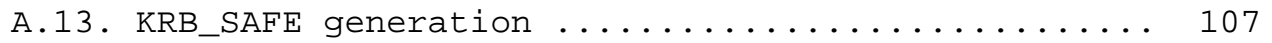

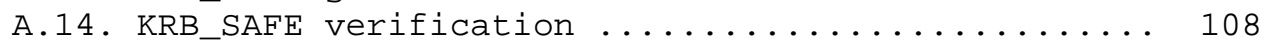




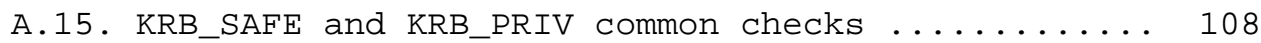

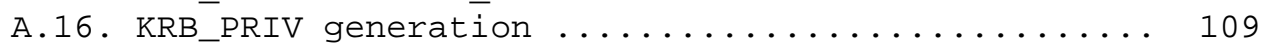

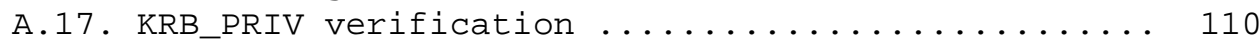

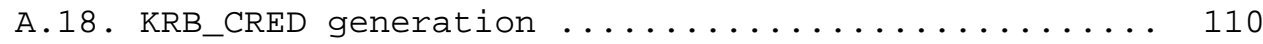

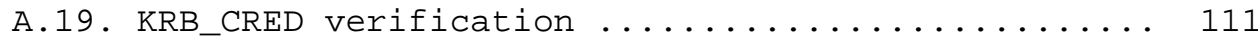

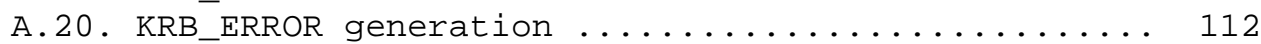

\section{Introduction}

Kerberos provides a means of verifying the identities of principals, (e.g., a workstation user or a network server) on an open (unprotected) network. This is accomplished without relying on authentication by the host operating system, without basing trust on host addresses, without requiring physical security of all the hosts on the network, and under the assumption that packets traveling along the network can be read, modified, and inserted at will. (Note, however, that many applications use Kerberos' functions only upon the initiation of a stream-based network connection, and assume the absence of any "hijackers" who might subvert such a connection. Such use implicitly trusts the host addresses involved.) Kerberos performs authentication under these conditions as a trusted thirdparty authentication service by using conventional cryptography, i.e., shared secret key. (shared secret key - secret and private are often used interchangeably in the literature. In our usage, it takes two (or more) to share a secret, thus a shared DES key is a secret key. Something is only private when no one but its owner knows it. Thus, in public key cryptosystems, one has a public and a private key.)

The authentication process proceeds as follows: A client sends a request to the authentication server (AS) requesting "credentials" for a given server. The AS responds with these credentials, encrypted in the client's key. The credentials consist of 1) a "ticket" for the server and 2) a temporary encryption key (often called a "session key"). The client transmits the ticket (which contains the client's identity and a copy of the session key, all encrypted in the server's key) to the server. The session key (now shared by the client and server) is used to authenticate the client, and may optionally be used to authenticate the server. It may also be used to encrypt further communication between the two parties or to exchange a separate sub-session key to be used to encrypt further communication.

The implementation consists of one or more authentication servers running on physically secure hosts. The authentication servers maintain a database of principals (i.e., users and servers) and their secret keys. Code libraries provide encryption and implement the Kerberos protocol. In order to add authentication to its 
transactions, a typical network application adds one or two calls to the Kerberos library, which results in the transmission of the necessary messages to achieve authentication.

The Kerberos protocol consists of several sub-protocols (or exchanges). There are two methods by which a client can ask a Kerberos server for credentials. In the first approach, the client sends a cleartext request for a ticket for the desired server to the AS. The reply is sent encrypted in the client's secret key. Usually this request is for a ticket-granting ticket (TGT) which can later be used with the ticket-granting server (TGS). In the second method, the client sends a request to the TGS. The client sends the TGT to the TGS in the same manner as if it were contacting any other application server which requires Kerberos credentials. The reply is encrypted in the session key from the TGT.

Once obtained, credentials may be used to verify the identity of the principals in a transaction, to ensure the integrity of messages exchanged between them, or to preserve privacy of the messages. The application is free to choose whatever protection may be necessary.

To verify the identities of the principals in a transaction, the client transmits the ticket to the server. Since the ticket is sent "in the clear" (parts of it are encrypted, but this encryption doesn't thwart replay) and might be intercepted and reused by an attacker, additional information is sent to prove that the message was originated by the principal to whom the ticket was issued. This information (called the authenticator) is encrypted in the session key, and includes a timestamp. The timestamp proves that the message was recently generated and is not a replay. Encrypting the authenticator in the session key proves that it was generated by a party possessing the session key. Since no one except the requesting principal and the server know the session key (it is never sent over the network in the clear) this guarantees the identity of the client.

The integrity of the messages exchanged between principals can also be guaranteed using the session key (passed in the ticket and contained in the credentials). This approach provides detection of both replay attacks and message stream modification attacks. It is accomplished by generating and transmitting a collision-proof checksum (elsewhere called a hash or digest function) of the client's message, keyed with the session key. Privacy and integrity of the messages exchanged between principals can be secured by encrypting the data to be passed using the session key passed in the ticket, and contained in the credentials.

The authentication exchanges mentioned above require read-only access to the Kerberos database. Sometimes, however, the entries in the 
database must be modified, such as when adding new principals or changing a principal's key. This is done using a protocol between a client and a third Kerberos server, the Kerberos Administration Server (KADM). The administration protocol is not described in this document. There is also a protocol for maintaining multiple copies of the Kerberos database, but this can be considered an implementation detail and may vary to support different database technologies.

\subsection{Cross-Realm Operation}

The Kerberos protocol is designed to operate across organizational boundaries. A client in one organization can be authenticated to a server in another. Each organization wishing to run a Kerberos server establishes its own "realm". The name of the realm in which a client is registered is part of the client's name, and can be used by the end-service to decide whether to honor a request.

By establishing "inter-realm" keys, the administrators of two realms can allow a client authenticated in the local realm to use its authentication remotely (Of course, with appropriate permission the client could arrange registration of a separately-named principal in a remote realm, and engage in normal exchanges with that realm's services. However, for even small numbers of clients this becomes cumbersome, and more automatic methods as described here are necessary). The exchange of inter-realm keys (a separate key may be used for each direction) registers the ticket-granting service of each realm as a principal in the other realm. A client is then able to obtain a ticket-granting ticket for the remote realm's ticketgranting service from its local realm. When that ticket-granting ticket is used, the remote ticket-granting service uses the interrealm key (which usually differs from its own normal TGS key) to decrypt the ticket-granting ticket, and is thus certain that it was issued by the client's own TGS. Tickets issued by the remote ticketgranting service will indicate to the end-service that the client was authenticated from another realm.

A realm is said to communicate with another realm if the two realms share an inter-realm key, or if the local realm shares an inter-realm key with an intermediate realm that communicates with the remote realm. An authentication path is the sequence of intermediate realms that are transited in communicating from one realm to another.

Realms are typically organized hierarchically. Each realm shares a key with its parent and a different key with each child. If an inter-realm key is not directly shared by two realms, the hierarchical organization allows an authentication path to be easily constructed. If a hierarchical organization is not used, it may be necessary to consult some database in order to construct an 
authentication path between realms.

Although realms are typically hierarchical, intermediate realms may be bypassed to achieve cross-realm authentication through alternate authentication paths (these might be established to make communication between two realms more efficient). It is important for the end-service to know which realms were transited when deciding how much faith to place in the authentication process. To facilitate this decision, a field in each ticket contains the names of the realms that were involved in authenticating the client.

\subsection{Environmental assumptions}

Kerberos imposes a few assumptions on the environment in which it can properly function:

$+\quad$ "Denial of service" attacks are not solved with Kerberos. There are places in these protocols where an intruder intruder can prevent an application from participating in the proper authentication steps. Detection and solution of such attacks (some of which can appear to be not-uncommon "normal" failure modes for the system) is usually best left to the human administrators and users.

$+\quad$ Principals must keep their secret keys secret. If an intruder somehow steals a principal's key, it will be able to masquerade as that principal or impersonate any server to the legitimate principal.

+ "Password guessing" attacks are not solved by Kerberos. If a user chooses a poor password, it is possible for an attacker to successfully mount an offline dictionary attack by repeatedly attempting to decrypt, with successive entries from a dictionary, messages obtained which are encrypted under a key derived from the user's password.

+ Each host on the network must have a clock which is "loosely synchronized" to the time of the other hosts; this synchronization is used to reduce the bookkeeping needs of application servers when they do replay detection. The degree of "looseness" can be configured on a per-server basis. If the clocks are synchronized over the network, the clock synchronization protocol must itself be secured from network attackers.

$+\quad$ Principal identifiers are not recycled on a short-term basis. A typical mode of access control will use access control lists (ACLs) to grant permissions to particular principals. If a 
stale ACL entry remains for a deleted principal and the principal identifier is reused, the new principal will inherit rights specified in the stale ACL entry. By not re-using principal identifiers, the danger of inadvertent access is removed.

\subsection{Glossary of terms}

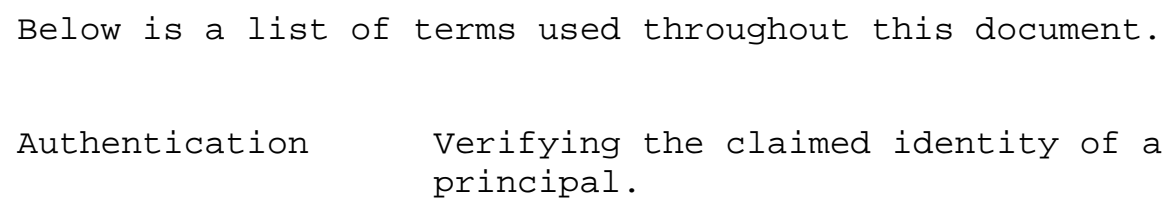

Authenticator

A record containing information that can be shown to have been recently generated using the session key known only by the client and server.

Authorization

The process of determining whether a client may use a service, which objects the client is allowed to access, and the type of access allowed for each.

Capability

A token that grants the bearer permission to access an object or service. In Kerberos, this might be a ticket whose use is restricted by the contents of the authorization data field, but which lists no network addresses, together with the session key necessary to use the ticket. 
Ciphertext

Client

Credentials

$\mathrm{KDC}$

Kerberos

Plaintext

Principal
The output of an encryption function. Encryption transforms plaintext into ciphertext.

A process that makes use of a network service on behalf of a user. Note that in some cases a Server may itself be a client of some other server (e.g., a print server may be a client of a file server).

A ticket plus the secret session key necessary to successfully use that ticket in an authentication exchange.

Key Distribution Center, a network service that supplies tickets and temporary session keys; or an instance of that service or the host on which it runs. The KDC services both initial ticket and ticket-granting ticket requests. The initial ticket portion is sometimes referred to as the Authentication Server (or service). The ticket-granting ticket portion is sometimes referred to as the ticket-granting server (or service).

Aside from the 3-headed dog guarding Hades, the name given to Project Athena's authentication service, the protocol used by that service, or the code used to implement the authentication service.

The input to an encryption function or the output of a decryption function. Decryption transforms ciphertext into plaintext.

A uniquely named client or server instance that participates in a network communication. 
Principal identifier The name used to uniquely identify each different principal.

Seal

Secret key

Server

Service

Session key

Sub-session key

Ticket

Kohl \& Neuman
To encipher a record containing several fields in such a way that the fields cannot be individually replaced without either knowledge of the encryption key or leaving evidence of tampering.

An encryption key shared by a principal and the KDC, distributed outside the bounds of the system, with a long lifetime. In the case of a human user's principal, the secret key is derived from a password.

A particular Principal which provides a resource to network clients.

A resource provided to network clients; often provided by more than one server (for example, remote file service).

A temporary encryption key used between two principals, with a lifetime limited to the duration of a single login "session".

A temporary encryption key used between two principals, selected and exchanged by the principals using the session key, and with a lifetime limited to the duration of a single association.

A record that helps a client authenticate itself to a server; it contains the client's identity, a session key, a timestamp, and other information, all sealed using the server's secret key. It only serves to authenticate a client when presented along with a fresh Authenticator. 
2. Ticket flag uses and requests

Each Kerberos ticket contains a set of flags which are used to indicate various attributes of that ticket. Most flags may be requested by a client when the ticket is obtained; some are automatically turned on and off by a Kerberos server as required. The following sections explain what the various flags mean, and gives examples of reasons to use such a flag.

\subsection{Initial and pre-authenticated tickets}

The INITIAL flag indicates that a ticket was issued using the AS protocol and not issued based on a ticket-granting ticket. Application servers that want to require the knowledge of a client's secret key (e.g., a passwordchanging program) can insist that this flag be set in any tickets they accept, and thus be assured that the client's key was recently presented to the application client.

The PRE-AUTHENT and HW-AUTHENT flags provide addition information about the initial authentication, regardless of whether the current ticket was issued directly (in which case INITIAL will also be set) or issued on the basis of a ticket-granting ticket (in which case the INITIAL flag is clear, but the PRE-AUTHENT and HW-AUTHENT flags are carried forward from the ticket-granting ticket).

\subsection{Invalid tickets}

The INVALID flag indicates that a ticket is invalid. Application servers must reject tickets which have this flag set. A postdated ticket will usually be issued in this form. Invalid tickets must be validated by the KDC before use, by presenting them to the KDC in a TGS request with the VALIDATE option specified. The KDC will only validate tickets after their starttime has passed. The validation is required so that postdated tickets which have been stolen before their starttime can be rendered permanently invalid (through a hotlist mechanism).

\subsection{Renewable tickets}

Applications may desire to hold tickets which can be valid for long periods of time. However, this can expose their credentials to potential theft for equally long periods, and those stolen credentials would be valid until the expiration time of the ticket(s). Simply using shortlived tickets and obtaining new ones periodically would require the client to have long-term access to its secret key, an even greater risk. Renewable tickets can be used to mitigate the consequences of theft. Renewable tickets have two "expiration times": the first is when the current instance of the 
ticket expires, and the second is the latest permissible value for an individual expiration time. An application client must periodically (i.e., before it expires) present a renewable ticket to the KDC, with the RENEW option set in the KDC request. The KDC will issue a new ticket with a new session key and a later expiration time. All other fields of the ticket are left unmodified by the renewal process. When the latest permissible expiration time arrives, the ticket expires permanently. At each renewal, the KDC may consult a hot-list to determine if the ticket had been reported stolen since its last renewal; it will refuse to renew such stolen tickets, and thus the usable lifetime of stolen tickets is reduced.

The RENEWABLE flag in a ticket is normally only interpreted by the ticket-granting service (discussed below in section 3.3). It can usually be ignored by application servers. However, some particularly careful application servers may wish to disallow renewable tickets.

If a renewable ticket is not renewed by its expiration time, the KDC will not renew the ticket. The RENEWABLE flag is reset by default, but a client may request it be set by setting the RENEWABLE option in the KRB_AS_REQ message. If it is set, then the renew-till field in the ticket contains the time after which the ticket may not be renewed.

\subsection{Postdated tickets}

Applications may occasionally need to obtain tickets for use much later, e.g., a batch submission system would need tickets to be valid at the time the batch job is serviced. However, it is dangerous to hold valid tickets in a batch queue, since they will be on-line longer and more prone to theft. Postdated tickets provide a way to obtain these tickets from the KDC at job submission time, but to leave them "dormant" until they are activated and validated by a further request of the $\mathrm{KDC}$. If a ticket theft were reported in the interim, the $\mathrm{KDC}$ would refuse to validate the ticket, and the thief would be foiled.

The MAY-POSTDATE flag in a ticket is normally only interpreted by the ticket-granting service. It can be ignored by application servers. This flag must be set in a ticket-granting ticket in order to issue a postdated ticket based on the presented ticket. It is reset by default; it may be requested by a client by setting the ALLOWPOSTDATE option in the KRB_AS_REQ message. This flag does not allow a client to obtain a postdated ticket-granting ticket; postdated ticket-granting tickets can only by obtained by requesting the postdating in the KRB_AS_REQ message. The life (endtime-starttime) of a postdated ticket will be the remaining life of the ticket- 
granting ticket at the time of the request, unless the RENEWABLE option is also set, in which case it can be the full life (endtimestarttime) of the ticket-granting ticket. The KDC may limit how far in the future a ticket may be postdated.

The POSTDATED flag indicates that a ticket has been postdated. The application server can check the authtime field in the ticket to see when the original authentication occurred. Some services may choose to reject postdated tickets, or they may only accept them within a certain period after the original authentication. When the KDC issues a POSTDATED ticket, it will also be marked as INVALID, so that the application client must present the ticket to the KDC to be validated before use.

\subsection{Proxiable and proxy tickets}

At times it may be necessary for a principal to allow a service to perform an operation on its behalf. The service must be able to take on the identity of the client, but only for a particular purpose. A principal can allow a service to take on the principal's identity for a particular purpose by granting it a proxy.

The PROXIABLE flag in a ticket is normally only interpreted by the ticket-granting service. It can be ignored by application servers. When set, this flag tells the ticket-granting server that it is oK to issue a new ticket (but not a ticket-granting ticket) with a different network address based on this ticket. This flag is set by default.

This flag allows a client to pass a proxy to a server to perform a remote request on its behalf, e.g., a print service client can give the print server a proxy to access the client's files on a particular file server in order to satisfy a print request.

In order to complicate the use of stolen credentials, Kerberos tickets are usually valid from only those network addresses specifically included in the ticket (It is permissible to request or issue tickets with no network addresses specified, but we do not recommend it). For this reason, a client wishing to grant a proxy must request a new ticket valid for the network address of the service to be granted the proxy.

The PROXY flag is set in a ticket by the TGS when it issues a proxy ticket. Application servers may check this flag and require additional authentication from the agent presenting the proxy in order to provide an audit trail. 


\subsection{Forwardable tickets}

Authentication forwarding is an instance of the proxy case where the service is granted complete use of the client's identity. An example where it might be used is when a user logs in to a remote system and wants authentication to work from that system as if the login were local.

The FORWARDABLE flag in a ticket is normally only interpreted by the ticket-granting service. It can be ignored by application servers. The FORWARDABLE flag has an interpretation similar to that of the PROXIABLE flag, except ticket-granting tickets may also be issued with different network addresses. This flag is reset by default, but users may request that it be set by setting the FORWARDABLE option in the AS request when they request their initial ticket-granting ticket.

This flag allows for authentication forwarding without requiring the user to enter a password again. If the flag is not set, then authentication forwarding is not permitted, but the same end result can still be achieved if the user engages in the AS exchange with the requested network addresses and supplies a password.

The FORWARDED flag is set by the TGS when a client presents a ticket with the FORWARDABLE flag set and requests it be set by specifying the FORWARDED KDC option and supplying a set of addresses for the new ticket. It is also set in all tickets issued based on tickets with the FORWARDED flag set. Application servers may wish to process FORWARDED tickets differently than non-FORWARDED tickets.

\subsection{Other KDC options}

There are two additional options which may be set in a client's request of the KDC. The RENEWABLE-OK option indicates that the client will accept a renewable ticket if a ticket with the requested life cannot otherwise be provided. If a ticket with the requested life cannot be provided, then the KDC may issue a renewable ticket with a renew-till equal to the the requested endtime. The value of the renew-till field may still be adjusted by site-determined limits or limits imposed by the individual principal or server.

The ENC-TKT-IN-SKEY option is honored only by the ticket-granting service. It indicates that the to-be-issued ticket for the end server is to be encrypted in the session key from the additional ticket-granting ticket provided with the request. See section 3.3 .3 for specific details. 


\section{Message Exchanges}

The following sections describe the interactions between network clients and servers and the messages involved in those exchanges.

3.1. The Authentication Service Exchange

$$
\text { Summary }
$$

$\begin{array}{lll}\text { Message direction } & \text { Message type } & \text { Section } \\ \text { 1. Client to Kerberos } & \text { KRB_AS_REQ } & 5.4 .1 \\ \text { 2. Kerberos to client } & \text { KRB_AS_REP or } & 5.4 .2 \\ & \text { KRB_ERROR } & 5.9 .1\end{array}$

The Authentication Service (AS) Exchange between the client and the Kerberos Authentication Server is usually initiated by a client when it wishes to obtain authentication credentials for a given server but currently holds no credentials. The client's secret key is used for encryption and decryption. This exchange is typically used at the initiation of a login session, to obtain credentials for a TicketGranting Server, which will subsequently be used to obtain credentials for other servers (see section 3.3) without requiring further use of the client's secret key. This exchange is also used to request credentials for services which must not be mediated through the Ticket-Granting Service, but rather require a principal's secret key, such as the password-changing service. (The passwordchanging request must not be honored unless the requester can provide the old password (the user's current secret key). Otherwise, it would be possible for someone to walk up to an unattended session and change another user's password.) This exchange does not by itself provide any assurance of the the identity of the user. (To authenticate a user logging on to a local system, the credentials obtained in the AS exchange may first be used in a TGS exchange to obtain credentials for a local server. Those credentials must then be verified by the local server through successful completion of the client/Server exchange.)

The exchange consists of two messages: KRB_AS_REQ from the client to Kerberos, and KRB_AS_REP or KRB_ERROR in reply. The formats for these messages are described in sections 5.4.1, 5.4.2, and 5.9.1.

In the request, the client sends (in cleartext) its own identity and the identity of the server for which it is requesting credentials. The response, KRB_AS_REP, contains a ticket for the client to present to the server, and a session key that will be shared by the client and the server. The session key and additional information are encrypted in the client's secret key. The KRB_AS_REP message contains information which can be used to detect replays, and to 
associate it with the message to which it replies. Various errors can occur; these are indicated by an error response (KRB_ERROR) instead of the KRB_AS_REP response. The error message is not encrypted. The KRB_ERROR message also contains information which can be used to associate it with the message to which it replies. The lack of encryption in the KRB_ERROR message precludes the ability to detect replays or fabrications of such messages.

In the normal case the authentication server does not know whether the client is actually the principal named in the request. It simply sends a reply without knowing or caring whether they are the same. This is acceptable because nobody but the principal whose identity was given in the request will be able to use the reply. Its critical information is encrypted in that principal's key. The initial request supports an optional field that can be used to pass additional information that might be needed for the initial exchange. This field may be used for preauthentication if desired, but the mechanism is not currently specified.

\subsubsection{Generation of KRB_AS_REQ message}

The client may specify a number of options in the initial request. Among these options are whether preauthentication is to be performed; whether the requested ticket is to be renewable, proxiable, or forwardable; whether it should be postdated or allow postdating of derivative tickets; and whether a renewable ticket will be accepted in lieu of a non-renewable ticket if the requested ticket expiration date cannot be satisfied by a nonrenewable ticket (due to configuration constraints; see section 4). See section A.1 for pseudocode.

The client prepares the KRB_AS_REQ message and sends it to the KDC.

\subsubsection{Receipt of KRB_AS_REQ message}

If all goes well, processing the KRB_AS_REQ message will result in the creation of a ticket for the client to present to the server. The format for the ticket is described in section 5.3.1. The contents of the ticket are determined as follows.

\subsubsection{Generation of KRB_AS_REP message}

The authentication server looks up the client and server principals named in the KRB_AS_REQ in its database, extracting their respective keys. If required, the server pre-authenticates the request, and if the pre-authentication check fails, an error message with the code KDC_ERR_PREAUTH_FAILED is returned. If the server cannot accommodate the requested encryption type, an error message with code 
KDC_ERR_ETYPE_NOSUPP is returned. Otherwise it generates a "random" session key ("Random" means that, among other things, it should be impossible to guess the next session key based on knowledge of past session keys. This can only be achieved in a pseudo-random number generator if it is based on cryptographic principles. It would be more desirable to use a truly random number generator, such as one based on measurements of random physical phenomena.).

If the requested start time is absent or indicates a time in the past, then the start time of the ticket is set to the authentication server's current time. If it indicates a time in the future, but the POSTDATED option has not been specified, then the error KDC_ERR_CANNOT_POSTDATE is returned. Otherwise the requested start time is checked against the policy of the local realm (the administrator might decide to prohibit certain types or ranges of postdated tickets), and if acceptable, the ticket's start time is set as requested and the INVALID flag is set in the new ticket. The postdated ticket must be validated before use by presenting it to the KDC after the start time has been reached.

The expiration time of the ticket will be set to the minimum of the following:

+The expiration time (endtime) requested in the KRB_AS_REQ message.

+The ticket's start time plus the maximum allowable lifetime associated with the client principal (the authentication server's database includes a maximum ticket lifetime field in each principal's record; see section 4).

+The ticket's start time plus the maximum allowable lifetime associated with the server principal.

the ticket's start time plus the maximum lifetime set by the policy of the local realm.

If the requested expiration time minus the start time (as determined above) is less than a site-determined minimum lifetime, an error message with code KDC_ERR_NEVER_VALID is returned. If the requested expiration time for the ticket exceeds what was determined as above, and if the "RENEWABLE-OK" option was requested, then the "RENEWABLE" flag is set in the new ticket, and the renew-till value is set as if the "RENEWABLE" option were requested (the field and option names are described fully in section 5.4.1). If the RENEWABLE option has been requested or if the RENEWABLE-OK option has been set and a renewable ticket is to be issued, then the renew-till field is set to the minimum of: 
+Its requested value.

The start time of the ticket plus the minimum of the two maximum renewable lifetimes associated with the principals' database entries.

The start time of the ticket plus the maximum renewable lifetime set by the policy of the local realm.

The flags field of the new ticket will have the following options set if they have been requested and if the policy of the local realm allows: FORWARDABLE, MAY-POSTDATE, POSTDATED, PROXIABLE, RENEWABLE. If the new ticket is postdated (the start time is in the future), its INVALID flag will also be set.

If all of the above succeed, the server formats a KRB_AS_REP message (see section 5.4.2), copying the addresses in the request into the caddr of the response, placing any required pre-authentication data into the padata of the response, and encrypts the ciphertext part in the client's key using the requested encryption method, and sends it to the client. See section A.2 for pseudocode.

\subsubsection{Generation of KRB_ERROR message}

Several errors can occur, and the Authentication Server responds by returning an error message, KRB_ERROR, to the client, with the error-code and e-text fields set to appropriate values. The error message contents and details are described in section 5.9.1.

\subsubsection{Receipt of KRB_AS_REP message}

If the reply message type is KRB_AS_REP, then the client verifies that the cname and crealm fields in the cleartext portion of the reply match what it requested. If any padata fields are present, they may be used to derive the proper secret key to decrypt the message. The client decrypts the encrypted part of the response using its secret key, verifies that the nonce in the encrypted part matches the nonce it supplied in its request (to detect replays). It also verifies that the sname and srealm in the response match those in the request, and that the host address field is also correct. It then stores the ticket, session key, start and expiration times, and other information for later use. The key-expiration field from the encrypted part of the response may be checked to notify the user of impending key expiration (the client program could then suggest remedial action, such as a password change). See section A. 3 for pseudocode.

Proper decryption of the KRB_AS_REP message is not sufficient to 
verify the identity of the user; the user and an attacker could cooperate to generate a KRB_AS_REP format message which decrypts properly but is not from the proper KDC. If the host wishes to verify the identity of the user, it must require the user to present application credentials which can be verified using a securely-stored secret key. If those credentials can be verified, then the identity of the user can be assured.

\subsubsection{Receipt of KRB_ERROR message}

If the reply message type is KRB_ERROR, then the client interprets it as an error and performs whatever application-specific tasks are necessary to recover.

\subsection{The Client/Server Authentication Exchange}

\section{Summary}

$\begin{array}{lll}\text { Message direction } & \text { Message type } & \text { Section } \\ \text { Client to Application server } & \text { KRB_AP_REQ } & 5.5 .1 \\ \text { [optional] Application server to client } & \text { KRB_AP_REP or } & 5.5 .2 \\ & \text { KRB_ERROR } & 5.9 .1\end{array}$

The client/server authentication (CS) exchange is used by network applications to authenticate the client to the server and vice versa. The client must have already acquired credentials for the server using the AS or TGS exchange.

\subsubsection{The KRB_AP_REQ message}

The KRB_AP_REQ contains authentication information which should be part of the first message in an authenticated transaction. It contains a ticket, an authenticator, and some additional bookkeeping information (see section 5.5.1 for the exact format). The ticket by itself is insufficient to authenticate a client, since tickets are passed across the network in cleartext(Tickets contain both an encrypted and unencrypted portion, so cleartext here refers to the entire unit, which can be copied from one message and replayed in another without any cryptographic skill.), so the authenticator is used to prevent invalid replay of tickets by proving to the server that the client knows the session key of the ticket and thus is entitled to use it. The KRB_AP_REQ message is referred to elsewhere as the "authentication header."

\subsubsection{Generation of a KRB_AP_REQ message}

When a client wishes to initiate authentication to a server, it obtains (either through a credentials cache, the AS exchange, or the 
TGS exchange) a ticket and session key for the desired service. The client may re-use any tickets it holds until they expire. The client then constructs a new Authenticator from the the system time, its name, and optionally an application specific checksum, an initial sequence number to be used in KRB_SAFE or KRB_PRIV messages, and/or a session subkey to be used in negotiations for a session key unique to this particular session. Authenticators may not be re-used and will be rejected if replayed to a server (Note that this can make applications based on unreliable transports difficult to code correctly, if the transport might deliver duplicated messages. In such cases, a new authenticator must be generated for each retry.). If a sequence number is to be included, it should be randomly chosen so that even after many messages have been exchanged it is not likely to collide with other sequence numbers in use.

The client may indicate a requirement of mutual authentication or the use of a session-key based ticket by setting the appropriate flag(s) in the ap-options field of the message.

The Authenticator is encrypted in the session key and combined with the ticket to form the KRB_AP_REQ message which is then sent to the end server along with any additional application-specific information. See section A.9 for pseudocode.

\subsubsection{Receipt of KRB_AP_REQ message}

Authentication is based on the server's current time of day (clocks must be loosely synchronized), the authenticator, and the ticket. Several errors are possible. If an error occurs, the server is expected to reply to the client with a KRB_ERROR message. This message may be encapsulated in the application protocol if its "raw" form is not acceptable to the protocol. The format of error messages is described in section 5.9.1.

The algorithm for verifying authentication information is as follows. If the message type is not $K R B \_A P \_R E Q$, the server returns the KRB_AP_ERR_MSG_TYPE error. If the key version indicated by the Ticket in the KRB_AP_REQ is not one the server can use (e.g., it indicates an old key, and the server no longer possesses a copy of the old key), the KRB_AP_ERR_BADKEYVER error is returned. If the USESESSION-KEY flag is set in the ap-options field, it indicates to the server that the ticket is encrypted in the session key from the server's ticket-granting ticket rather than its secret key (This is used for user-to-user authentication as described in [6]). Since it is possible for the server to be registered in multiple realms, with different keys in each, the srealm field in the unencrypted portion of the ticket in the KRB_AP_REQ is used to specify which secret key the server should use to decrypt that ticket. The KRB_AP_ERR_NOKEY 
error code is returned if the server doesn't have the proper key to decipher the ticket.

The ticket is decrypted using the version of the server's key specified by the ticket. If the decryption routines detect a modification of the ticket (each encryption system must provide safeguards to detect modified ciphertext; see section 6), the KRB_AP_ERR_BAD_INTEGRITY error is returned (chances are good that different keys were used to encrypt and decrypt).

The authenticator is decrypted using the session key extracted from the decrypted ticket. If decryption shows it to have been modified, the KRB_AP_ERR_BAD_INTEGRITY error is returned. The name and realm of the client from the ticket are compared against the same fields in the authenticator. If they don't match, the KRB_AP_ERR_BADMATCH error is returned (they might not match, for example, if the wrong session key was used to encrypt the authenticator). The addresses in the ticket (if any) are then searched for an address matching the operating-system reported address of the client. If no match is found or the server insists on ticket addresses but none are present in the ticket, the KRB_AP_ERR_BADADDR error is returned.

If the local (server) time and the client time in the authenticator differ by more than the allowable clock skew (e.g., 5 minutes), the KRB_AP_ERR_SKEW error is returned. If the server name, along with the client name, time and microsecond fields from the Authenticator match any recently-seen such tuples, the KRB_AP_ERR_REPEAT error is returned (Note that the rejection here is restricted to authenticators from the same principal to the same server. Other client principals communicating with the same server principal should not be have their authenticators rejected if the time and microsecond fields happen to match some other client's authenticator.). The server must remember any authenticator presented within the allowable clock skew, so that a replay attempt is guaranteed to fail. If a server loses track of any authenticator presented within the allowable clock skew, it must reject all requests until the clock skew interval has passed. This assures that any lost or re-played authenticators will fall outside the allowable clock skew and can no longer be successfully replayed (If this is not done, an attacker could conceivably record the ticket and authenticator sent over the network to a server, then disable the client's host, pose as the disabled host, and replay the ticket and authenticator to subvert the authentication.). If a sequence number is provided in the authenticator, the server saves it for later use in processing KRB_SAFE and/or KRB_PRIV messages. If a subkey is present, the server either saves it for later use or uses it to help generate its own choice for a subkey to be returned in a KRB_AP_REP message. 
The server computes the age of the ticket: local (server) time minus the start time inside the Ticket. If the start time is later than the current time by more than the allowable clock skew or if the INVALID flag is set in the ticket, the KRB_AP_ERR_TKT_NYV error is returned. Otherwise, if the current time is later than end time by more than the allowable clock skew, the KRB_AP_ERR_TKT_EXPIRED error is returned.

If all these checks succeed without an error, the server is assured that the client possesses the credentials of the principal named in the ticket and thus, the client has been authenticated to the server. See section A.10 for pseudocode.

\subsubsection{Generation of a KRB_AP_REP message}

Typically, a client's request will include both the authentication information and its initial request in the same message, and the server need not explicitly reply to the KRB_AP_REQ. However, if mutual authentication (not only authenticating the client to the server, but also the server to the client) is being performed, the KRB_AP_REQ message will have MUTUAL-REQUIRED set in its ap-options field, and a KRB_AP_REP message is required in response. As with the error message, this message may be encapsulated in the application protocol if its "raw" form is not acceptable to the application's protocol. The timestamp and microsecond field used in the reply must be the client's timestamp and microsecond field (as provided in the authenticator). [Note: In the Kerberos version 4 protocol, the timestamp in the reply was the client's timestamp plus one. This is not necessary in version 5 because version 5 messages are formatted in such a way that it is not possible to create the reply by judicious message surgery (even in encrypted form) without knowledge of the appropriate encryption keys.] If a sequence number is to be included, it should be randomly chosen as described above for the authenticator. A subkey may be included if the server desires to negotiate a different subkey. The KRB_AP_REP message is encrypted in the session key extracted from the ticket. See section A.11 for pseudocode.

\subsubsection{Receipt of KRB_AP_REP message}

If a KRB_AP_REP message is returned, the client uses the session key from the credentials obtained for the server (Note that for encrypting the KRB_AP_REP message, the sub-session key is not used, even if present in the Authenticator.) to decrypt the message, and verifies that the timestamp and microsecond fields match those in the Authenticator it sent to the server. If they match, then the client is assured that the server is genuine. The sequence number and subkey (if present) are retained for later use. See section A.12 for 
pseudocode.

3.2.6. Using the encryption key

After the KRB_AP_REQ/KRB_AP_REP exchange has occurred, the client and server share an encryption key which can be used by the application. The "true session key" to be used for KRB_PRIV, KRB_SAFE, or other application-specific uses may be chosen by the application based on the subkeys in the KRB_AP_REP message and the authenticator (Implementations of the protocol may wish to provide routines to choose subkeys based on session keys and random numbers and to orchestrate a negotiated key to be returned in the KRB_AP_REP message.). In some cases, the use of this session key will be implicit in the protocol; in others the method of use must be chosen from a several alternatives. We leave the protocol negotiations of how to use the key (e.g., selecting an encryption or checksum type) to the application programmer; the Kerberos protocol does not constrain the implementation options.

With both the one-way and mutual authentication exchanges, the peers should take care not to send sensitive information to each other without proper assurances. In particular, applications that require privacy or integrity should use the KRB_AP_REP or KRB_ERROR responses from the server to client to assure both client and server of their peer's identity. If an application protocol requires privacy of its messages, it can use the KRB_PRIV message (section 3.5). The KRB_SAFE message (section 3.4) can be used to assure integrity.

3.3. The Ticket-Granting Service (TGS) Exchange

\section{Summary}

$\begin{array}{lll}\text { Message direction } & \text { Message type } & \text { Section } \\ \text { 1. Client to Kerberos } & \text { KRB_TGS_REQ } & 5.4 .1 \\ \text { 2. Kerberos to client } & \text { KRB_TGS_REP or } & 5.4 .2 \\ & \text { KRB_ERROR } & 5.9 .1\end{array}$

The TGS exchange between a client and the Kerberos Ticket-Granting Server is initiated by a client when it wishes to obtain authentication credentials for a given server (which might be registered in a remote realm), when it wishes to renew or validate an existing ticket, or when it wishes to obtain a proxy ticket. In the first case, the client must already have acquired a ticket for the Ticket-Granting Service using the AS exchange (the ticket-granting ticket is usually obtained when a client initially authenticates to the system, such as when a user logs in). The message format for the TGS exchange is almost identical to that for the AS exchange. The primary difference is that encryption and decryption in the TGS 
exchange does not take place under the client's key. Instead, the session key from the ticket-granting ticket or renewable ticket, or sub-session key from an Authenticator is used. As is the case for all application servers, expired tickets are not accepted by the TGS, so once a renewable or ticket-granting ticket expires, the client must use a separate exchange to obtain valid tickets.

The TGS exchange consists of two messages: A request (KRB_TGS_REQ) from the client to the Kerberos Ticket-Granting Server, and a reply (KRB_TGS_REP or KRB_ERROR). The KRB_TGS_REQ message includes information authenticating the client plus a request for credentials. The authentication information consists of the authentication header (KRB_AP_REQ) which includes the client's previously obtained ticketgranting, renewable, or invalid ticket. In the ticket-granting ticket and proxy cases, the request may include one or more of: a list of network addresses, a collection of typed authorization data to be sealed in the ticket for authorization use by the application server, or additional tickets (the use of which are described later). The TGS reply (KRB_TGS_REP) contains the requested credentials, encrypted in the session key from the ticket-granting ticket or renewable ticket, or if present, in the subsession key from the Authenticator (part of the authentication header). The KRB_ERROR message contains an error code and text explaining what went wrong. The KRB_ERROR message is not encrypted. The KRB_TGS_REP message contains information which can be used to detect replays, and to associate it with the message to which it replies. The KRB_ERROR message also contains information which can be used to associate it with the message to which it replies, but the lack of encryption in the KRB_ERROR message precludes the ability to detect replays or fabrications of such messages.

\subsubsection{Generation of KRB_TGS_REQ message}

Before sending a request to the ticket-granting service, the client must determine in which realm the application server is registered [Note: This can be accomplished in several ways. It might be known beforehand (since the realm is part of the principal identifier), or it might be stored in a nameserver. Presently, however, this information is obtained from a configuration file. If the realm to be used is obtained from a nameserver, there is a danger of being spoofed if the nameservice providing the realm name is not authenticated. This might result in the use of a realm which has been compromised, and would result in an attacker's ability to compromise the authentication of the application server to the client.]. If the client does not already possess a ticket-granting ticket for the appropriate realm, then one must be obtained. This is first attempted by requesting a ticket-granting ticket for the destination realm from the local Kerberos server (using the 
KRB_TGS_REQ message recursively). The Kerberos server may return a TGT for the desired realm in which case one can proceed. Alternatively, the Kerberos server may return a TGT for a realm which is "closer" to the desired realm (further along the standard hierarchical path), in which case this step must be repeated with a Kerberos server in the realm specified in the returned TGT. If neither are returned, then the request must be retried with a Kerberos server for a realm higher in the hierarchy. This request will itself require a ticket-granting ticket for the higher realm which must be obtained by recursively applying these directions.

Once the client obtains a ticket-granting ticket for the appropriate realm, it determines which Kerberos servers serve that realm, and contacts one. The list might be obtained through a configuration file or network service; as long as the secret keys exchanged by realms are kept secret, only denial of service results from a false Kerberos server.

As in the AS exchange, the client may specify a number of options in the KRB_TGS_REQ message. The client prepares the KRB_TGS_REQ message, providing an authentication header as an element of the padata field, and including the same fields as used in the KRB_AS_REQ message along with several optional fields: the enc-authorizationdata field for application server use and additional tickets required by some options.

In preparing the authentication header, the client can select a subsession key under which the response from the Kerberos server will be encrypted (If the client selects a sub-session key, care must be taken to ensure the randomness of the selected subsession key. One approach would be to generate a random number and XOR it with the session key from the ticket-granting ticket.). If the sub-session key is not specified, the session key from the ticket-granting ticket will be used. If the enc-authorization-data is present, it must be encrypted in the sub-session key, if present, from the authenticator portion of the authentication header, or if not present in the session key from the ticket-granting ticket.

Once prepared, the message is sent to a Kerberos server for the destination realm. See section A.5 for pseudocode.

\subsubsection{Receipt of KRB_TGS_REQ message}

The KRB_TGS_REQ message is processed in a manner similar to the KRB_AS_REQ message, but there are many additional checks to be performed. First, the Kerberos server must determine which server the accompanying ticket is for and it must select the appropriate key to decrypt it. For a normal KRB_TGS_REQ message, it will be for the 
ticket granting service, and the TGS's key will be used. If the TGT was issued by another realm, then the appropriate inter-realm key must be used. If the accompanying ticket is not a ticket granting ticket for the current realm, but is for an application server in the current realm, the RENEW, VALIDATE, or PROXY options are specified in the request, and the server for which a ticket is requested is the server named in the accompanying ticket, then the KDC will decrypt the ticket in the authentication header using the key of the server for which it was issued. If no ticket can be found in the padata field, the KDC_ERR_PADATA_TYPE_NOSUPP error is returned.

Once the accompanying ticket has been decrypted, the user-supplied checksum in the Authenticator must be verified against the contents of the request, and the message rejected if the checksums do not match (with an error code of KRB_AP_ERR_MODIFIED) or if the checksum is not keyed or not collision-proof (with an error code of KRB_AP_ERR_INAPP_CKSUM). If the checksum type is not supported, the KDC_ERR_SUMTYPE_NOSUPP error is returned. If the authorization-data are present, they are decrypted using the sub-session key from the Authenticator.

If any of the decryptions indicate failed integrity checks, the KRB_AP_ERR_BAD_INTEGRITY error is returned.

\subsubsection{Generation of KRB_TGS_REP message}

The KRB_TGS_REP message shares its format with the KRB_AS_REP (KRB_KDC_REP), but with its type field set to KRB_TGS_REP. The detailed specification is in section 5.4.2.

The response will include a ticket for the requested server. The Kerberos database is queried to retrieve the record for the requested server (including the key with which the ticket will be encrypted). If the request is for a ticket granting ticket for a remote realm, and if no key is shared with the requested realm, then the Kerberos server will select the realm "closest" to the requested realm with which it does share a key, and use that realm instead. This is the only case where the response from the KDC will be for a different server than that requested by the client.

By default, the address field, the client's name and realm, the list of transited realms, the time of initial authentication, the expiration time, and the authorization data of the newly-issued ticket will be copied from the ticket-granting ticket (TGT) or renewable ticket. If the transited field needs to be updated, but the transited type is not supported, the KDC_ERR_TRTYPE_NOSUPP error is returned. 
If the request specifies an endtime, then the endtime of the new ticket is set to the minimum of (a) that request, (b) the endtime from the TGT, and (c) the starttime of the TGT plus the minimum of the maximum life for the application server and the maximum life for the local realm (the maximum life for the requesting principal was already applied when the TGT was issued). If the new ticket is to be a renewal, then the endtime above is replaced by the minimum of (a) the value of the renew_till field of the ticket and (b) the starttime for the new ticket plus the life (endtimestarttime) of the old ticket.

If the FORWARDED option has been requested, then the resulting ticket will contain the addresses specified by the client. This option will only be honored if the FORWARDABLE flag is set in the TGT. The PROXY option is similar; the resulting ticket will contain the addresses specified by the client. It will be honored only if the PROXIABLE flag in the TGT is set. The PROXY option will not be honored on requests for additional ticket-granting tickets.

If the requested start time is absent or indicates a time in the past, then the start time of the ticket is set to the authentication server's current time. If it indicates a time in the future, but the POSTDATED option has not been specified or the MAY-POSTDATE flag is not set in the TGT, then the error KDC_ERR_CANNOT_POSTDATE is returned. Otherwise, if the ticket-granting ticket has the MAYPOSTDATE flag set, then the resulting ticket will be postdated and the requested starttime is checked against the policy of the local realm. If acceptable, the ticket's start time is set as requested, and the INVALID flag is set. The postdated ticket must be validated before use by presenting it to the KDC after the starttime has been reached. However, in no case may the starttime, endtime, or renewtill time of a newly-issued postdated ticket extend beyond the renew-till time of the ticket-granting ticket.

If the ENC-TKT-IN-SKEY option has been specified and an additional ticket has been included in the request, the KDC will decrypt the additional ticket using the key for the server to which the additional ticket was issued and verify that it is a ticket-granting ticket. If the name of the requested server is missing from the request, the name of the client in the additional ticket will be used. Otherwise the name of the requested server will be compared to the name of the client in the additional ticket and if different, the request will be rejected. If the request succeeds, the session key from the additional ticket will be used to encrypt the new ticket that is issued instead of using the key of the server for which the new ticket will be used (This allows easy implementation of user-touser authentication [6], which uses ticket-granting ticket session keys in lieu of secret server keys in situations where such secret 
keys could be easily compromised.) .

If the name of the server in the ticket that is presented to the KDC as part of the authentication header is not that of the ticketgranting server itself, and the server is registered in the realm of the KDC, If the RENEW option is requested, then the KDC will verify that the RENEWABLE flag is set in the ticket and that the renew_till time is still in the future. If the VALIDATE option is rqeuested, the KDC will check that the starttime has passed and the INVALID flag is set. If the PROXY option is requested, then the KDC will check that the PROXIABLE flag is set in the ticket. If the tests succeed, the KDC will issue the appropriate new ticket.

Whenever a request is made to the ticket-granting server, the presented ticket(s) is(are) checked against a hot-list of tickets which have been canceled. This hot-list might be implemented by storing a range of issue dates for "suspect tickets"; if a presented ticket had an authtime in that range, it would be rejected. In this way, a stolen ticket-granting ticket or renewable ticket cannot be used to gain additional tickets (renewals or otherwise) once the theft has been reported. Any normal ticket obtained before it was reported stolen will still be valid (because they require no interaction with the $\mathrm{KDC}$ ), but only until their normal expiration time.

The ciphertext part of the response in the KRB_TGS_REP message is encrypted in the sub-session key from the Authenticator, if present, or the session key key from the ticket-granting ticket. It is not encrypted using the client's secret key. Furthermore, the client's key's expiration date and the key version number fields are left out since these values are stored along with the client's database record, and that record is not needed to satisfy a request based on a ticket-granting ticket. See section A.6 for pseudocode.

\subsubsection{Encoding the transited field}

If the identity of the server in the TGT that is presented to the KDC as part of the authentication header is that of the ticket-granting service, but the TGT was issued from another realm, the KDC will look up the inter-realm key shared with that realm and use that key to decrypt the ticket. If the ticket is valid, then the KDC will honor the request, subject to the constraints outlined above in the section describing the AS exchange. The realm part of the client's identity will be taken from the ticket-granting ticket. The name of the realm that issued the ticket-granting ticket will be added to the transited field of the ticket to be issued. This is accomplished by reading the transited field from the ticket-granting ticket (which is treated as an unordered set of realm names), adding the new realm to the set, 
then constructing and writing out its encoded (shorthand) form (this may involve a rearrangement of the existing encoding).

Note that the ticket-granting service does not add the name of its own realm. Instead, its responsibility is to add the name of the previous realm. This prevents a malicious Kerberos server from intentionally leaving out its own name (it could, however, omit other realms' names).

The names of neither the local realm nor the principal's realm are to be included in the transited field. They appear elsewhere in the ticket and both are known to have taken part in authenticating the principal. Since the endpoints are not included, both local and single-hop inter-realm authentication result in a transited field that is empty.

Because the name of each realm transited is added to this field, it might potentially be very long. To decrease the length of this field, its contents are encoded. The initially supported encoding is optimized for the normal case of inter-realm communication: a

hierarchical arrangement of realms using either domain or $\mathrm{x} .500$ style realm names. This encoding (called DOMAIN-X500-COMPRESS) is now described.

Realm names in the transited field are separated by a ",". The ",", "\", trailing "."s, and leading spaces (" ") are special characters, and if they are part of a realm name, they must be quoted in the transited field by preceding them with a "\".

A realm name ending with a "." is interpreted as being prepended to the previous realm. For example, we can encode traversal of EDU, MIT.EDU, ATHENA.MIT.EDU, WASHINGTON.EDU, and CS.WASHINGTON.EDU as:

"EDU, MIT . , ATHENA . , WASHINGTON . EDU, CS . " .

Note that if ATHENA.MIT.EDU, or CS.WASHINGTON.EDU were endpoints, that they would not be included in this field, and we would have:

"EDU, MIT . , WASHINGTON. EDU"

A realm name beginning with a "/" is interpreted as being appended to the previous realm (For the purpose of appending, the realm preceding the first listed realm is considered to be the null realm (")). If it is to stand by itself, then it should be preceded by a space (" ") . For example, we can encode traversal of /COM/HP/APOLLO, /COM/HP, /COM, and /COM/DEC as:

"/COM, /HP , /APOLLO, /COM/DEC". 
Like the example above, if /COM/HP/APOLLO and /COM/DEC are endpoints, they they would not be included in this field, and we would have:

\section{" /COM, / HP"}

A null subfield preceding or following a "," indicates that all realms between the previous realm and the next realm have been traversed (For the purpose of interpreting null subfields, the client's realm is considered to precede those in the transited field, and the server's realm is considered to follow them.). Thus, "," means that all realms along the path between the client and the server have been traversed. ",EDU, /COM," means that that all realms from the client's realm up to EDU (in a domain style hierarchy) have been traversed, and that everything from /COM down to the server's realm in an X.500 style has also been traversed. This could occur if the EDU realm in one hierarchy shares an inter-realm key directly with the /COM realm in another hierarchy.

\subsubsection{Receipt of KRB_TGS_REP message}

When the KRB_TGS_REP is received by the client, it is processed in the same manner as the KRB_AS_REP processing described above. The primary difference is that the ciphertext part of the response must be decrypted using the session key from the ticket-granting ticket rather than the client's secret key. See section A.7 for pseudocode.

\subsection{The KRB_SAFE Exchange}

The KRB_SAFE message may be used by clients requiring the ability to detect modifications of messages they exchange. It achieves this by including a keyed collisionproof checksum of the user data and some control information. The checksum is keyed with an encryption key (usually the last key negotiated via subkeys, or the session key if no negotiation has occured).

\subsubsection{Generation of a KRB_SAFE message}

When an application wishes to send a KRB_SAFE message, it collects its data and the appropriate control information and computes a checksum over them. The checksum algorithm should be some sort of keyed one-way hash function (such as the RSA-MD5-DES checksum algorithm specified in section 6.4.5, or the DES MAC), generated using the sub-session key if present, or the session key. Different algorithms may be selected by changing the checksum type in the message. Unkeyed or non-collision-proof checksums are not suitable for this use.

The control information for the KRB_SAFE message includes both a 
timestamp and a sequence number. The designer of an application using the KRB_SAFE message must choose at least one of the two mechanisms. This choice should be based on the needs of the application protocol.

Sequence numbers are useful when all messages sent will be received by one's peer. Connection state is presently required to maintain the session key, so maintaining the next sequence number should not present an additional problem.

If the application protocol is expected to tolerate lost messages without them being resent, the use of the timestamp is the appropriate replay detection mechanism. Using timestamps is also the appropriate mechanism for multi-cast protocols where all of one's peers share a common sub-session key, but some messages will be sent to a subset of one's peers.

After computing the checksum, the client then transmits the information and checksum to the recipient in the message format specified in section 5.6 .1 .

\subsubsection{Receipt of KRB_SAFE message}

When an application receives a KRB_SAFE message, it verifies it as follows. If any error occurs, an error code is reported for use by the application.

The message is first checked by verifying that the protocol version and type fields match the current version and KRB_SAFE, respectively. A mismatch generates a KRB_AP_ERR_BADVERSION or KRB_AP_ERR_MSG_TYPE error. The application verifies that the checksum used is a collisionproof keyed checksum, and if it is not, a KRB_AP_ERR_INAPP_CKSUM error is generated. The recipient verifies that the operating system's report of the sender's address matches the sender's address in the message, and (if a recipient address is specified or the recipient requires an address) that one of the recipient's addresses appears as the recipient's address in the message. A failed match for either case generates a KRB_AP_ERR_BADADDR error. Then the timestamp and usec and/or the sequence number fields are checked. If timestamp and usec are expected and not present, or they are present but not current, the KRB_AP_ERR_SKEW error is generated. If the server name, along with the client name, time and microsecond fields from the Authenticator match any recently-seen such tuples, the KRB_AP_ERR_REPEAT error is generated. If an incorrect sequence number is included, or a sequence number is expected but not present, the KRB_AP_ERR_BADORDER error is generated. If neither a timestamp and usec or a sequence number is present, a KRB_AP_ERR_MODIFIED error is generated. 
Finally, the checksum is computed over the data and control information, and if it doesn't match the received checksum, a KRB_AP_ERR_MODIFIED error is generated.

If all the checks succeed, the application is assured that the message was generated by its peer and was not modified in transit.

\subsection{The KRB_PRIV Exchange}

The KRB_PRIV message may be used by clients requiring confidentiality and the ability to detect modifications of exchanged messages. It achieves this by encrypting the messages and adding control information.

\subsubsection{Generation of a KRB_PRIV message}

When an application wishes to send a KRB_PRIV message, it collects its data and the appropriate control information (specified in section 5.7.1) and encrypts them under an encryption key (usually the last key negotiated via subkeys, or the session key if no negotiation has occured). As part of the control information, the client must choose to use either a timestamp or a sequence number (or both); see the discussion in section 3.4.1 for guidelines on which to use. After the user data and control information are encrypted, the client transmits the ciphertext and some "envelope" information to the recipient.

\subsubsection{Receipt of KRB_PRIV message}

When an application receives a KRB_PRIV message, it verifies it as follows. If any error occurs, an error code is reported for use by the application.

The message is first checked by verifying that the protocol version and type fields match the current version and KRB_PRIV, respectively. A mismatch generates a KRB_AP_ERR_BADVERSION or KRB_AP_ERR_MSG_TYPE error. The application then decrypts the ciphertext and processes the resultant plaintext. If decryption shows the data to have been modified, a KRB_AP_ERR_BAD_INTEGRITY error is generated. The recipient verifies that the operating system's report of the sender's address matches the sender's address in the message, and (if a recipient address is specified or the recipient requires an address) that one of the recipient's addresses appears as the recipient's address in the message. A failed match for either case generates a KRB_AP_ERR_BADADDR error. Then the timestamp and usec and/or the sequence number fields are checked. If timestamp and usec are expected and not present, or they are present but not current, the KRB_AP_ERR_SKEW error is generated. If the server name, along with 
the client name, time and microsecond fields from the Authenticator match any recently-seen such tuples, the KRB_AP_ERR_REPEAT error is generated. If an incorrect sequence number is included, or a sequence number is expected but not present, the KRB_AP_ERR_BADORDER error is generated. If neither a timestamp and usec or a sequence number is present, a KRB_AP_ERR_MODIFIED error is generated.

If all the checks succeed, the application can assume the message was generated by its peer, and was securely transmitted (without intruders able to see the unencrypted contents).

\subsection{The KRB_CRED Exchange}

The KRB_CRED message may be used by clients requiring the ability to send Kerberos credentials from one host to another. It achieves this by sending the tickets together with encrypted data containing the session keys and other information associated with the tickets.

\subsubsection{Generation of a KRB_CRED message}

When an application wishes to send a KRB_CRED message it first (using the KRB_TGS exchange) obtains credentials to be sent to the remote host. It then constructs a KRB_CRED message using the ticket or tickets so obtained, placing the session key needed to use each ticket in the key field of the corresponding KrbCredInfo sequence of the encrypted part of the the KRB_CRED message.

Other information associated with each ticket and obtained during the KRB_TGS exchange is also placed in the corresponding KrbCredInfo sequence in the encrypted part of the KRB_CRED message. The current time and, if specifically required by the application the nonce, saddress, and raddress fields, are placed in the encrypted part of the KRB_CRED message which is then encrypted under an encryption key previosuly exchanged in the KRB_AP exchange (usually the last key negotiated via subkeys, or the session key if no negotiation has occured).

\subsubsection{Receipt of KRB_CRED message}

When an application receives a KRB_CRED message, it verifies it. If any error occurs, an error code is reported for use by the application. The message is verified by checking that the protocol version and type fields match the current version and KRB_CRED, respectively. A mismatch generates a KRB_AP_ERR_BADVERSION or KRB_AP_ERR_MSG_TYPE error. The application then decrypts the ciphertext and processes the resultant plaintext. If decryption shows the data to have been modified, a KRB_AP_ERR_BAD_INTEGRITY error is generated. 
If present or required, the recipient verifies that the operating system's report of the sender's address matches the sender's address in the message, and that one of the recipient's addresses appears as the recipient's address in the message. A failed match for either case generates a KRB_AP_ERR_BADADDR error. The timestamp and usec fields (and the nonce field if required) are checked next. If the timestamp and usec are not present, or they are present but not current, the KRB_AP_ERR_SKEW error is generated.

If all the checks succeed, the application stores each of the new tickets in its ticket cache together with the session key and other information in the corresponding KrbCredInfo sequence from the encrypted part of the KRB_CRED message.

4. The Kerberos Database

The Kerberos server must have access to a database containing the principal identifiers and secret keys of principals to be authenticated (The implementation of the Kerberos server need not combine the database and the server on the same machine; it is feasible to store the principal database in, say, a network name service, as long as the entries stored therein are protected from disclosure to and modification by unauthorized parties. However, we recommend against such strategies, as they can make system management and threat analysis quite complex.).

4.1. Database contents

A database entry should contain at least the following fields:

Field Value

name $\quad$ Principal's identifier

key Principal's secret key

p_kvno Principal's key version

max_life Maximum lifetime for Tickets

max_renewable_life Maximum total lifetime for renewable Tickets

The name field is an encoding of the principal's identifier. The key field contains an encryption key. This key is the principal's secret key. (The key can be encrypted before storage under a Kerberos "master key" to protect it in case the database is compromised but the master key is not. In that case, an extra field must be added to indicate the master key version used, see below.) The p_kvno field is the key version number of the principal's secret key. The max_life field contains the maximum allowable lifetime (endtime - starttime) for any Ticket issued for this principal. The max_renewable_life 
field contains the maximum allowable total lifetime for any renewable Ticket issued for this principal. (See section 3.1 for a description of how these lifetimes are used in determining the lifetime of a given Ticket.)

A server may provide KDC service to several realms, as long as the database representation provides a mechanism to distinguish between principal records with identifiers which differ only in the realm name.

When an application server's key changes, if the change is routine (i.e., not the result of disclosure of the old key), the old key should be retained by the server until all tickets that had been issued using that key have expired. Because of this, it is possible for several keys to be active for a single principal. Ciphertext encrypted in a principal's key is always tagged with the version of the key that was used for encryption, to help the recipient find the proper key for decryption.

When more than one key is active for a particular principal, the principal will have more than one record in the Kerberos database. The keys and key version numbers will differ between the records (the rest of the fields may or may not be the same). Whenever Kerberos issues a ticket, or responds to a request for initial authentication, the most recent key (known by the Kerberos server) will be used for encryption. This is the key with the highest key version number.

\subsection{Additional fields}

Project Athena's KDC implementation uses additional fields in its database:

$\begin{array}{ll}\text { Field } & \text { Value } \\ \text { K_kvno } & \text { Kerberos' key version } \\ \text { expiration } & \text { Expiration date for entry } \\ \text { attributes } & \text { Bit field of attributes } \\ \text { mod_date } & \text { Timestamp of last modification } \\ \text { mod_name } & \text { Modifying principal's identifier }\end{array}$

The K_kvno field indicates the key version of the Kerberos master key under which the principal's secret key is encrypted.

After an entry's expiration date has passed, the KDC will return an error to any client attempting to gain tickets as or for the principal. (A database may want to maintain two expiration dates: one for the principal, and one for the principal's current key. This allows password aging to work independently of the principal's 
expiration date. However, due to the limited space in the responses, the KDC must combine the key expiration and principal expiration date into a single value called "key_exp", which is used as a hint to the user to take administrative action.)

The attributes field is a bitfield used to govern the operations involving the principal. This field might be useful in conjunction with user registration procedures, for site-specific policy implementations (Project Athena currently uses it for their user registration process controlled by the system-wide database service, Moira [7]), or to identify the "string to key" conversion algorithm used for a principal's key. (See the discussion of the padata field in section 5.4.2 for details on why this can be useful.) Other bits are used to indicate that certain ticket options should not be allowed in tickets encrypted under a principal's key (one bit each): Disallow issuing postdated tickets, disallow issuing forwardable tickets, disallow issuing tickets based on TGT authentication, disallow issuing renewable tickets, disallow issuing proxiable tickets, and disallow issuing tickets for which the principal is the server.

The mod_date field contains the time of last modification of the entry, and the mod_name field contains the name of the principal which last modified the entry.

\subsection{Frequently Changing Fields}

Some KDC implementations may wish to maintain the last time that a request was made by a particular principal. Information that might be maintained includes the time of the last request, the time of the last request for a ticket-granting ticket, the time of the last use of a ticket-granting ticket, or other times. This information can then be returned to the user in the last-req field (see section 5.2).

Other frequently changing information that can be maintained is the latest expiration time for any tickets that have been issued using each key. This field would be used to indicate how long old keys must remain valid to allow the continued use of outstanding tickets.

\subsection{Site Constants}

The KDC implementation should have the following configurable constants or options, to allow an administrator to make and enforce policy decisions:

+ The minimum supported lifetime (used to determine whether the KDC_ERR_NEVER_VALID error should be returned). This constant should reflect reasonable expectations of round-trip time to the 
KDC, encryption/decryption time, and processing time by the client and target server, and it should allow for a minimum "useful" lifetime.

+ The maximum allowable total (renewable) lifetime of a ticket (renew_till - starttime).

+ The maximum allowable lifetime of a ticket (endtime - starttime) .

+ Whether to allow the issue of tickets with empty address fields (including the ability to specify that such tickets may only be issued if the request specifies some authorization_data).

+ Whether proxiable, forwardable, renewable or post-datable tickets are to be issued.

5. Message Specifications

The following sections describe the exact contents and encoding of protocol messages and objects. The ASN.1 base definitions are presented in the first subsection. The remaining subsections specify the protocol objects (tickets and authenticators) and messages. Specification of encryption and checksum techniques, and the fields related to them, appear in section 6 .

\subsection{ASN.1 Distinguished Encoding Representation}

All uses of ASN.1 in Kerberos shall use the Distinguished Encoding Representation of the data elements as described in the $\mathrm{X} .509$ specification, section 8.7 [8].

\subsection{ASN.1 Base Definitions}

The following ASN.1 base definitions are used in the rest of this section. Note that since the underscore character (_) is not permitted in ASN.1 names, the hyphen (-) is used in its place for the purposes of ASN.1 names.

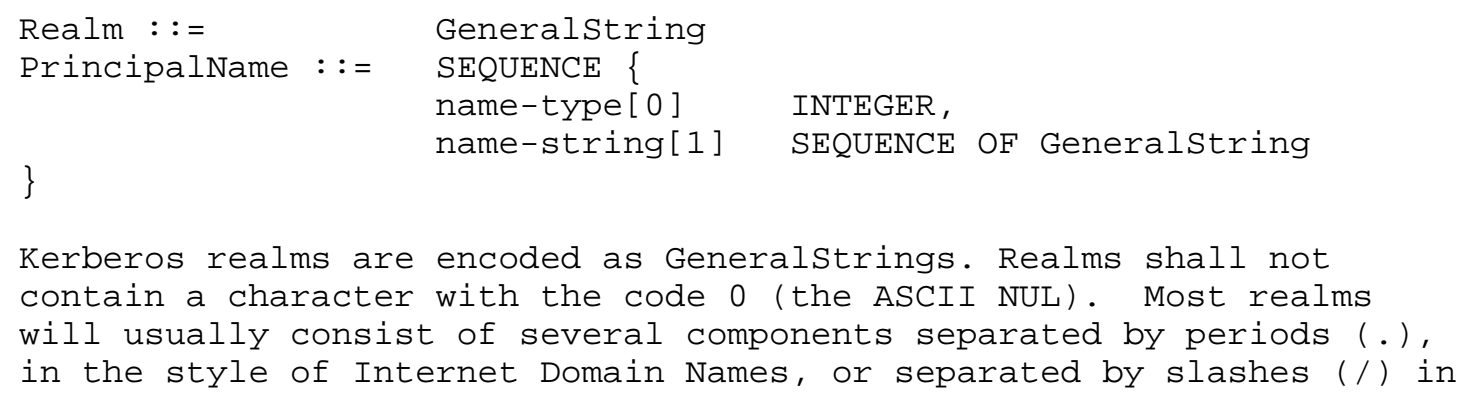




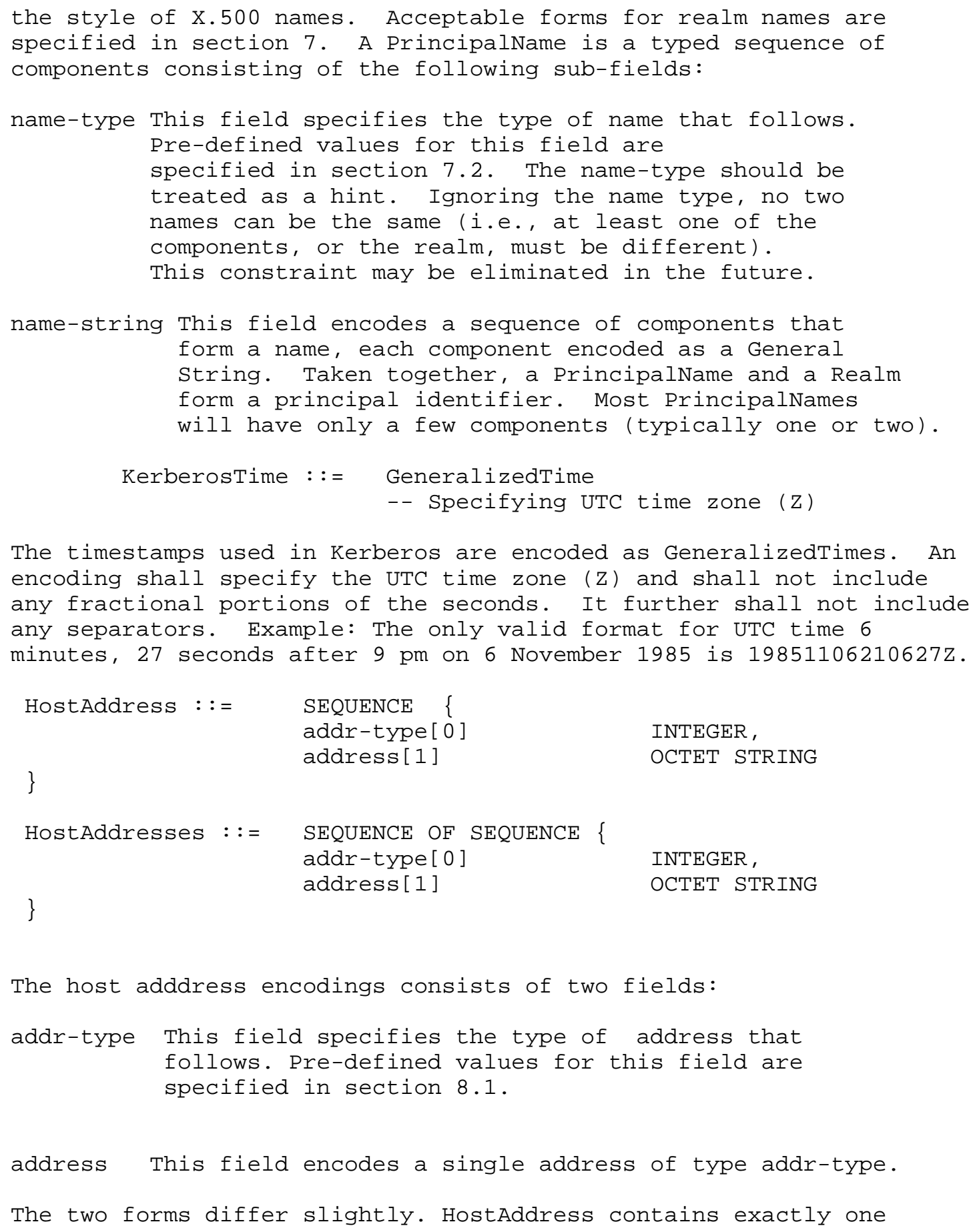




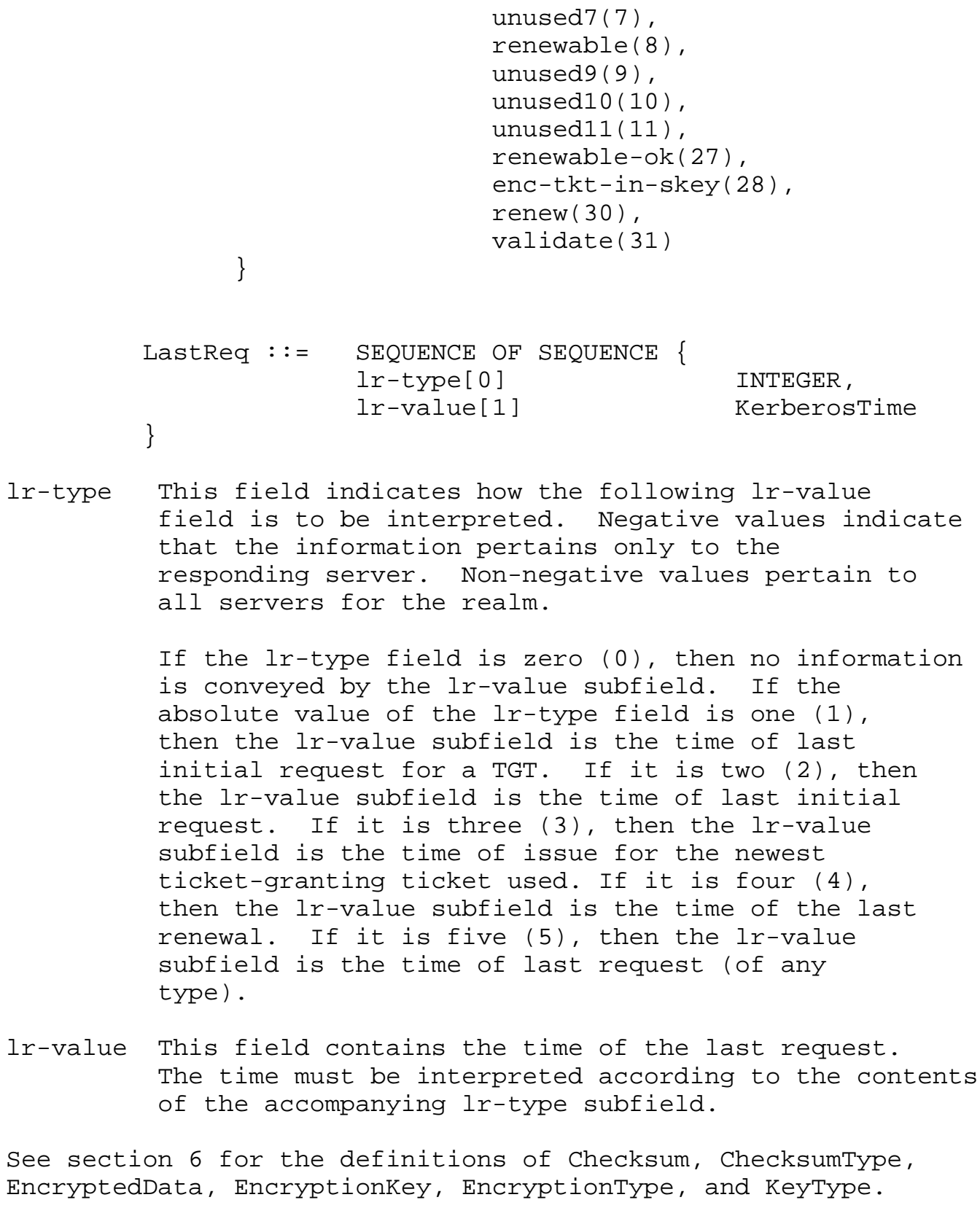




\subsection{Tickets and Authenticators}

This section describes the format and encryption parameters for tickets and authenticators. When a ticket or authenticator is included in a protocol message it is treated as an opaque object.

\subsubsection{Tickets}

A ticket is a record that helps a client authenticate to a service.

A Ticket contains the following information:

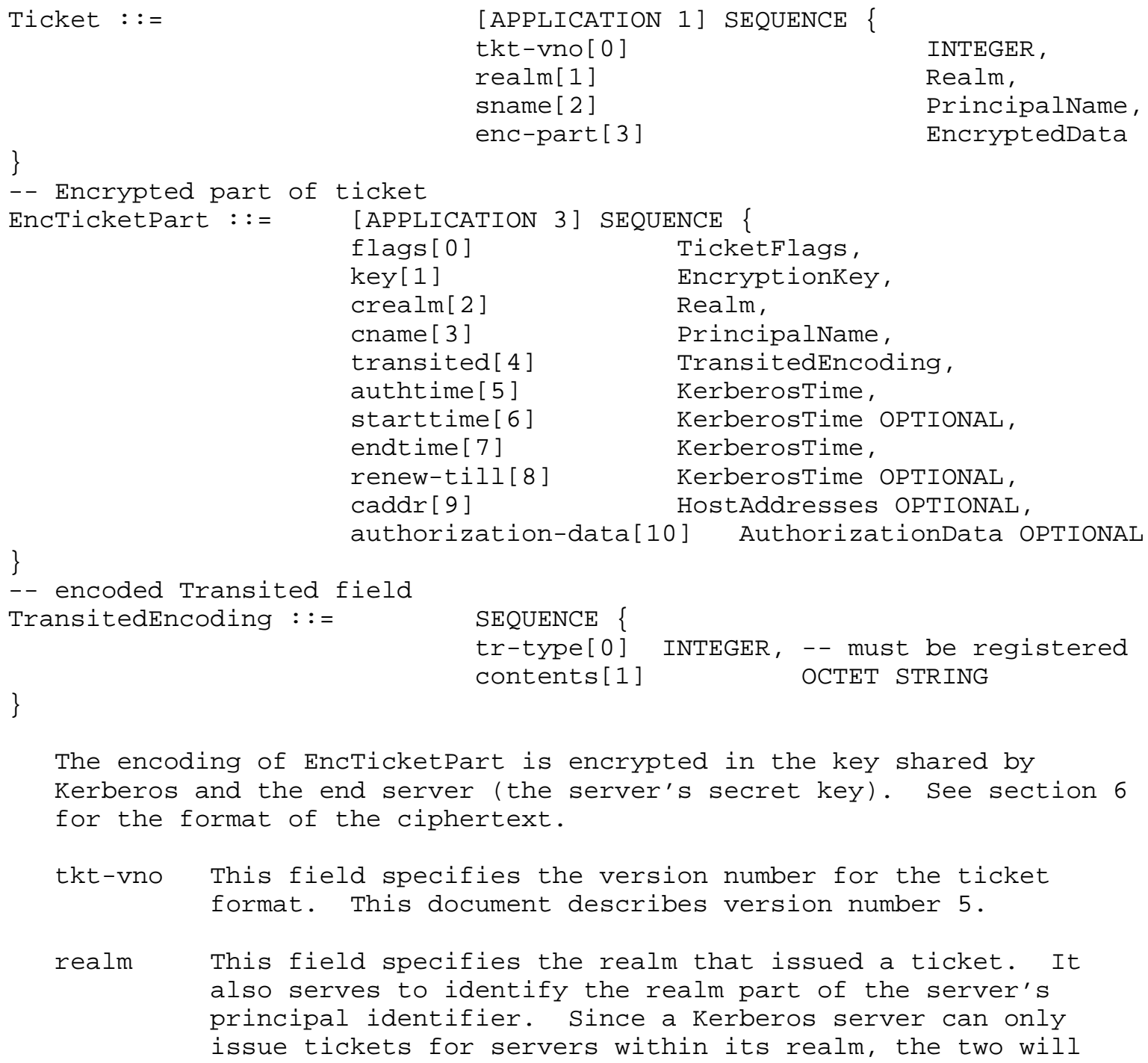




\begin{tabular}{|c|c|c|}
\hline sname & $\begin{array}{l}\text { This field specifies } t \\
\text { identity. }\end{array}$ & he name part of the server's \\
\hline enc-part & $\begin{array}{l}\text { This field holds the e } \\
\text { EncTicketPart sequence }\end{array}$ & ncrypted encoding of the \\
\hline \multirow[t]{6}{*}{ flags } & $\begin{array}{l}\text { This field indicates w } \\
\text { requested when the tic } \\
\text { where the selected opt } \\
\text { set ( } 1 \text { ), and the unsel } \\
\text { being reset (0). Bit } \\
\text { encoding of the bits i } \\
\text { flags are described in } \\
\text { meanings of the flags }\end{array}$ & $\begin{array}{l}\text { wich of various options were used or } \\
\text { aket was issued. It is a bit-field, } \\
\text { ions are indicated by the bit being } \\
\text { lected options and reserved fields } \\
0 \text { is the most significant bit. The } \\
\text { s specified in section } 5.2 \text {. The } \\
\text { more detail above in section } 2 \text {. The } \\
\text { are: }\end{array}$ \\
\hline & Bit (s) & Description \\
\hline & RESERVED & $\begin{array}{l}\text { Reserved for future expansion of this } \\
\text { field. }\end{array}$ \\
\hline & FORWARDABLE & $\begin{array}{l}\text { The FORWARDABLE flag is normally only } \\
\text { interpreted by the TGS, and can be } \\
\text { ignored by end servers. When set, } \\
\text { this flag tells the ticket-granting } \\
\text { server that it is ok to issue a new } \\
\text { ticket- granting ticket with a } \\
\text { different network address based on } \\
\text { the presented ticket. }\end{array}$ \\
\hline & FORWARDED & $\begin{array}{l}\text { When set, this flag indicates that } \\
\text { the ticket has either been forwarded } \\
\text { or was issued based on authentication } \\
\text { involving a forwarded ticket-granting } \\
\text { ticket. }\end{array}$ \\
\hline & PROXIABLE & $\begin{array}{l}\text { The PROXIABLE flag is normally only } \\
\text { interpreted by the TGS, and can be } \\
\text { ignored by end servers. The PROXIABLE } \\
\text { flag has an interpretation identical } \\
\text { to that of the FORWARDABLE flag, } \\
\text { except that the PROXIABLE flag tells } \\
\text { the ticket-granting server that only } \\
\text { non- ticket-granting tickets may be } \\
\text { issued with different network } \\
\text { addresses. }\end{array}$ \\
\hline
\end{tabular}


4
PROXY When set, this flag indicates that a ticket is a proxy.

MAY-POSTDATE The MAY-POSTDATE flag is normally only interpreted by the TGS, and can be ignored by end servers. This flag tells the ticket-granting server that a post- dated ticket may be issued based on this ticket-granting ticket.

POSTDATED This flag indicates that this ticket has been postdated. The end-service can check the authtime field to see when the original authentication occurred.

INVALID This flag indicates that a ticket is invalid, and it must be validated by the KDC before use. Application servers must reject tickets which have this flag set.

RENEWABLE The RENEWABLE flag is normally only interpreted by the TGS, and can usually be ignored by end servers (some particularly careful servers may wish to disallow renewable tickets). A renewable ticket can be used to obtain a replacement ticket that expires at a later date.

INITIAL This flag indicates that this ticket was issued using the AS protocol, and not issued based on a ticket-granting ticket.

PRE-AUTHENT This flag indicates that during initial authentication, the client was authenticated by the KDC before a ticket was issued. The strength of the preauthentication method is not indicated, but is acceptable to the $\mathrm{KDC}$.

HW-AUTHENT This flag indicates that the protocol employed for initial authentication required the use of hardware expected to be possessed solely by the named 


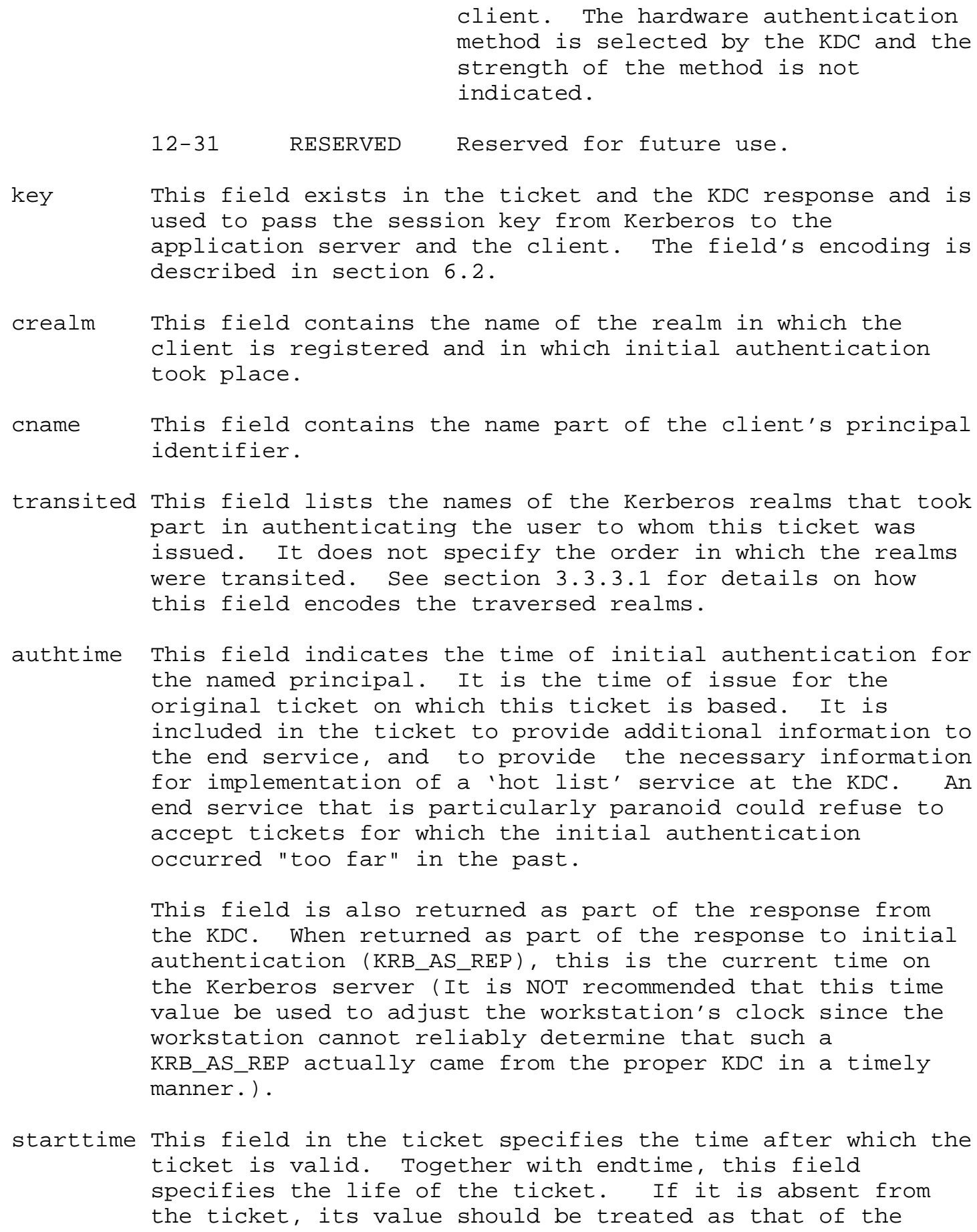


authtime field.

endtime This field contains the time after which the ticket will not be honored (its expiration time). Note that individual services may place their own limits on the life of a ticket and may reject tickets which have not yet expired. As such, this is really an upper bound on the expiration time for the ticket.

renew-till This field is only present in tickets that have the RENEWABLE flag set in the flags field. It indicates the maximum endtime that may be included in a renewal. It can be thought of as the absolute expiration time for the ticket, including all renewals.

caddr This field in a ticket contains zero (if omitted) or more (if present) host addresses. These are the addresses from which the ticket can be used. If there are no addresses, the ticket can be used from any location. The decision by the KDC to issue or by the end server to accept zeroaddress tickets is a policy decision and is left to the Kerberos and end-service administrators; they may refuse to issue or accept such tickets. The suggested and default policy, however, is that such tickets will only be issued or accepted when additional information that can be used to restrict the use of the ticket is included in the authorization_data field. Such a ticket is a capability.

Network addresses are included in the ticket to make it harder for an attacker to use stolen credentials. Because the session key is not sent over the network in cleartext, credentials can't be stolen simply by listening to the network; an attacker has to gain access to the session key (perhaps through operating system security breaches or a careless user's unattended session) to make use of stolen tickets.

It is important to note that the network address from which a connection is received cannot be reliably determined. Even if it could be, an attacker who has compromised the client's workstation could use the credentials from there. Including the network addresses only makes it more difficult, not impossible, for an attacker to walk off with stolen credentials and then use them from a "safe" location. 


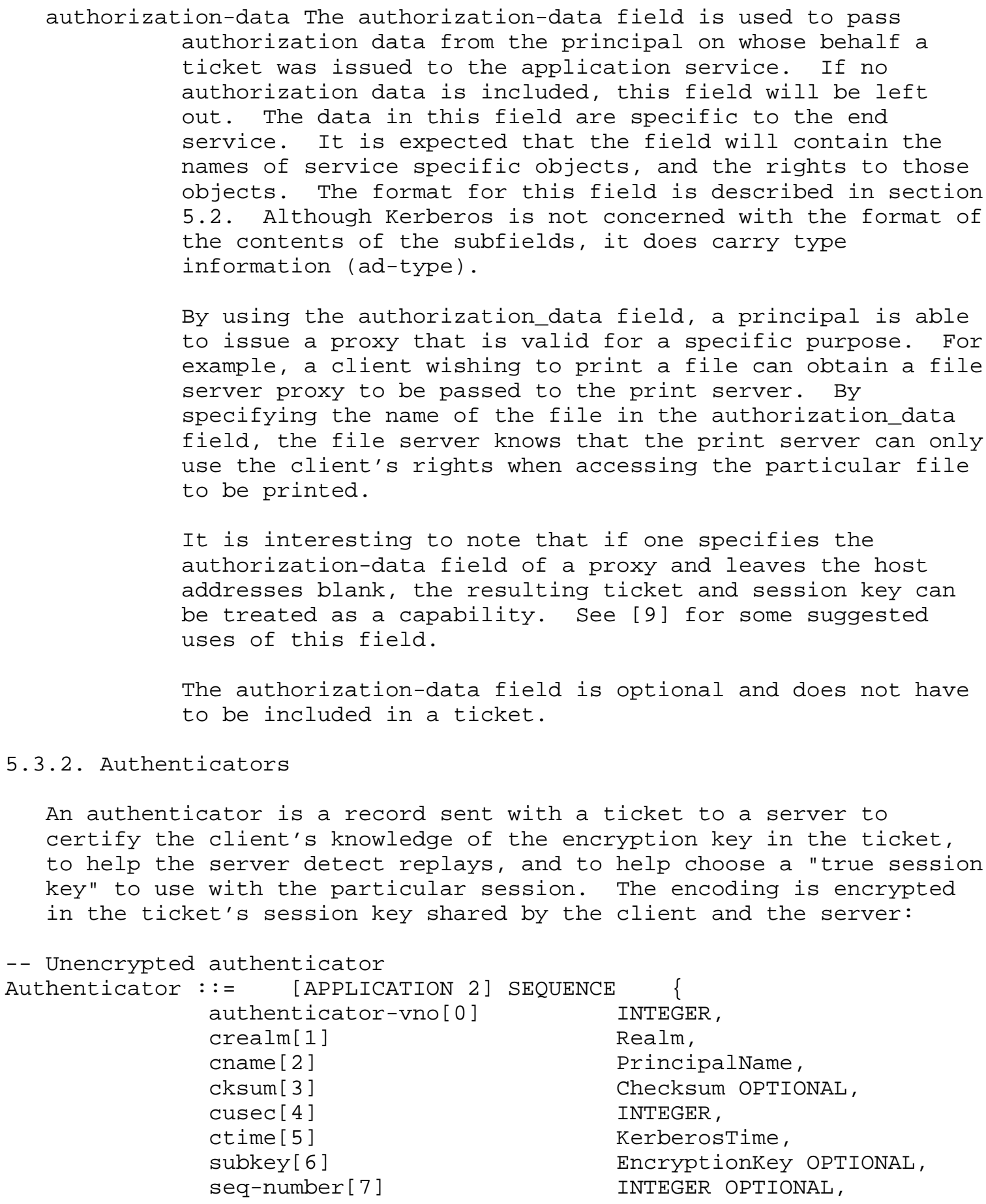

An authenticator is a record sent with a ticket to a server to certify the client's knowledge of the encryption key in the ticket, to help the server detect replays, and to help choose a "true session key" to use with the particular session. The encoding is encrypted in the ticket's session key shared by the client and the server:

-- Unencrypted authenticator

Authenticator : : $\quad$ [APPLICATION 2] SEQUENCE \{ authenticator-vno[0] INTEGER, crealm[1] Realm, cname [2] cksum [3] cusec [4] ctime [5] subkey [6] seq-number [7] PrincipalName, Checksum OPTIONAL, INTEGER, Kerberostime, Encryptionkey OPTIONAL, INTEGER OPTIONAL, 


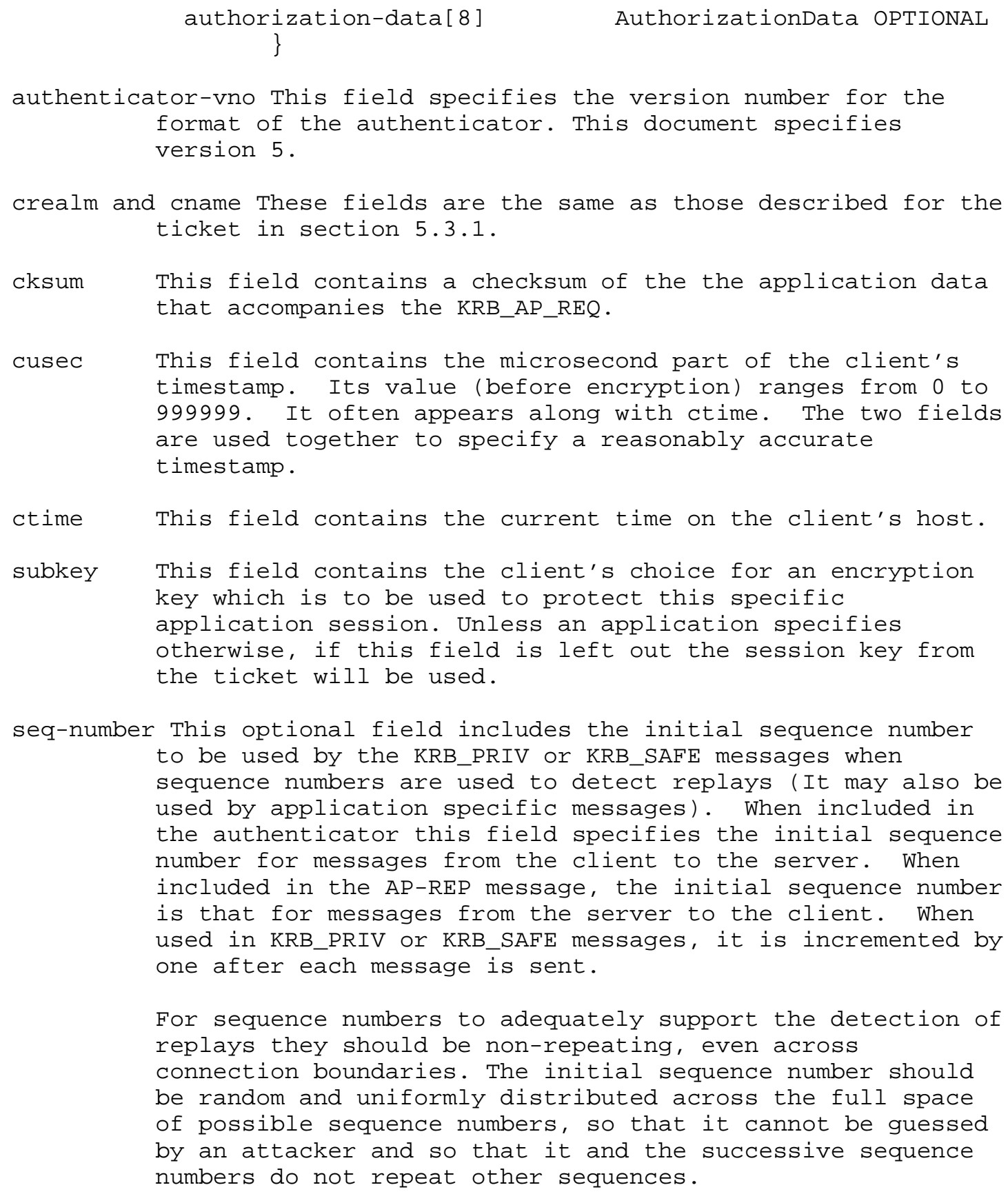


authorization-data This field is the same as described for the ticket in section 5.3.1. It is optional and will only appear when additional restrictions are to be placed on the use of a ticket, beyond those carried in the ticket itself.

5.4. Specifications for the AS and TGS exchanges

This section specifies the format of the messages used in exchange between the client and the Kerberos server. The format of possible error messages appears in section 5.9.1.

5.4.1. KRB_KDC_REQ definition

The KRB_KDC_REQ message has no type of its own. Instead, its type is one of KRB_AS_REQ or KRB_TGS_REQ depending on whether the request is for an initial ticket or an additional ticket. In either case, the message is sent from the client to the Authentication Server to request credentials for a service.

The message fields are:

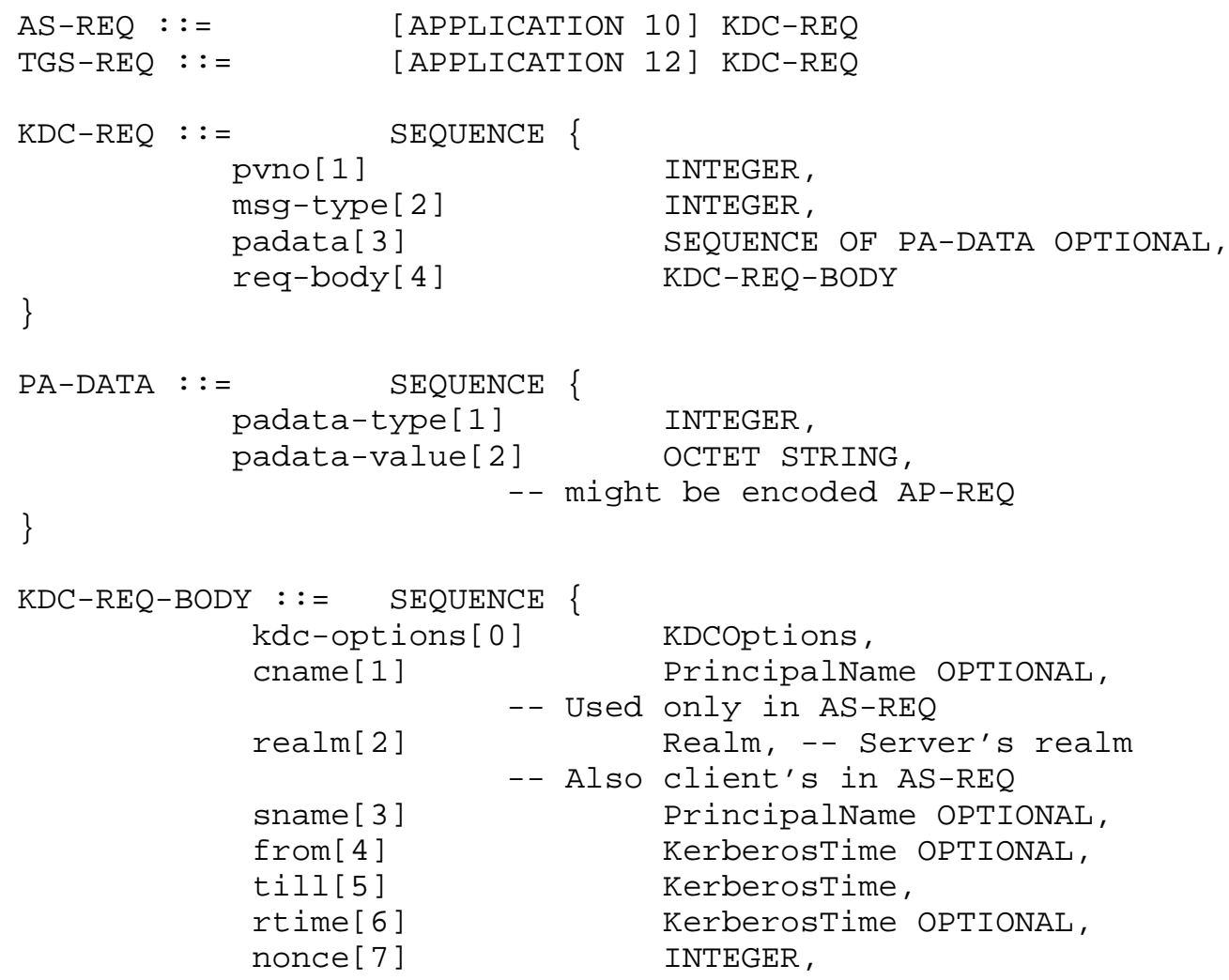

Kohl \& Neuman 


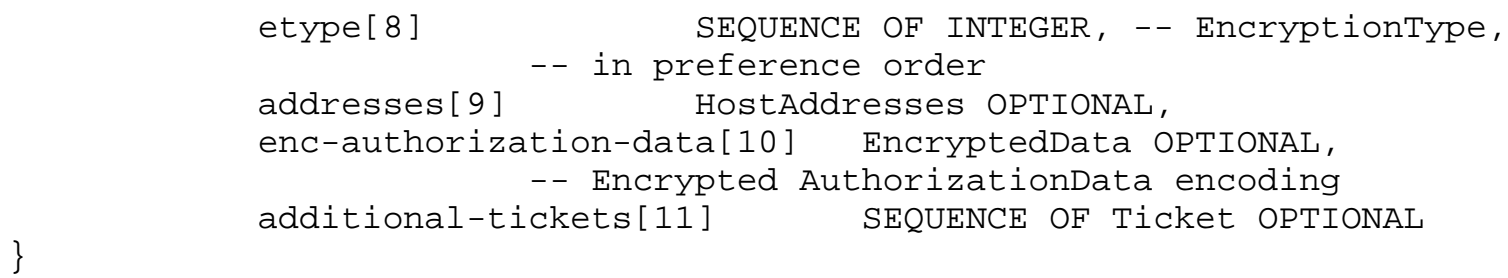


with patimestamp containing the client's time and pausec containing the microseconds which may be omitted if a client will not generate more than one request per second. The ciphertext (padata-value) consists of the PA-ENC-TS-ENC sequence, encrypted using the client's secret key.

The padata field can also contain information needed to help the KDC or the client select the key needed for generating or decrypting the response. This form of the padata is useful for supporting the use of certain "smartcards" with Kerberos. The details of such extensions are beyond the scope of this specification. See [10] for additional uses of this field.

padata-type The padata-type element of the padata field indicates the way that the padata-value element is to be interpreted. Negative values of padata-type are reserved for unregistered use; non-negative values are used for a registered interpretation of the element type.

req-body This field is a placeholder delimiting the extent of the remaining fields. If a checksum is to be calculated over the request, it is calculated over an encoding of the KDCREQ-BODY sequence which is enclosed within the req-body field.

kdc-options This field appears in the KRB_AS_REQ and KRB_TGS_REQ requests to the $\mathrm{KDC}$ and indicates the flags that the client wants set on the tickets as well as other information that is to modify the behavior of the KDC. Where appropriate, the name of an option may be the same as the flag that is set by that option. Although in most case, the bit in the options field will be the same as that in the flags field, this is not guaranteed, so it is not acceptable to simply copy the options field to the flags field. There are various checks that must be made before honoring an option anyway .

The kdc_options field is a bit-field, where the selected options are indicated by the bit being set (1), and the unselected options and reserved fields being reset (0). The encoding of the bits is specified in section 5.2. The options are described in more detail above in section 2 . The meanings of the options are: 


\begin{tabular}{|c|c|c|}
\hline Bit (s) & Name & Description \\
\hline 0 & RESERVED & $\begin{array}{l}\text { Reserved for future expansion of this } \\
\text { field. }\end{array}$ \\
\hline 1 & FORWARDABLE & $\begin{array}{l}\text { The FORWARDABLE option indicates that } \\
\text { the ticket to be issued is to have its } \\
\text { forwardable flag set. It may only be } \\
\text { set on the initial request, or in a } \\
\text { subsequent request if the ticket- } \\
\text { granting ticket on which it is based } \\
\text { is also forwardable. }\end{array}$ \\
\hline 2 & FORWARDED & $\begin{array}{l}\text { The FORWARDED option is only specified } \\
\text { in a request to the ticket-granting } \\
\text { server and will only be honored if the } \\
\text { ticket-granting ticket in the request } \\
\text { has its FORWARDABLE bit set. This } \\
\text { option indicates that this is a } \\
\text { request for forwarding. The } \\
\text { address (es) of the host from which the } \\
\text { resulting ticket is to be valid are } \\
\text { included in the addresses field of the } \\
\text { request. }\end{array}$ \\
\hline 3 & PROXIABLE & $\begin{array}{l}\text { The PROXIABLE option indicates that } \\
\text { the ticket to be issued is to have its } \\
\text { proxiable flag set. It may only be set } \\
\text { on the initial request, or in a } \\
\text { subsequent request if the ticket- } \\
\text { granting ticket on which it is based } \\
\text { is also proxiable. }\end{array}$ \\
\hline 4 & PROXY & $\begin{array}{l}\text { The PROXY option indicates that this } \\
\text { is a request for a proxy. This option } \\
\text { will only be honored if the ticket- } \\
\text { granting ticket in the request has its } \\
\text { PROXIABLE bit set. The address(es) of } \\
\text { the host from which the resulting } \\
\text { ticket is to be valid are included in } \\
\text { the addresses field of the request. }\end{array}$ \\
\hline 5 & ALLOW-POSTDA & $\begin{array}{l}\text { E The ALLOW-POSTDATE option indicates } \\
\text { that the ticket to be issued is to } \\
\text { have its MAY-POSTDATE flag set. It } \\
\text { may only be set on the initial } \\
\text { request, or in a subsequent request if }\end{array}$ \\
\hline
\end{tabular}


POSTDATED

the ticket-granting ticket on which it is based also has its MAY-POSTDATE flag set.

POSTDATED

The POSTDATED option indicates that this is a request for a postdated ticket. This option will only be honored if the ticket-granting ticket on which it is based has its MAYPOSTDATE flag set. The resulting ticket will also have its INVALID flag set, and that flag may be reset by a subsequent request to the $\mathrm{KDC}$ after the starttime in the ticket has been reached.

UNUSED

This option is presently unused.

The RENEWABLE option indicates that the ticket to be issued is to have its RENEWABLE flag set. It may only be set on the initial request, or when the ticket-granting ticket on which the request is based is also renewable. If this option is requested, then the rtime field in the request contains the desired absolute expiration time for the ticket.

9-26 RESERVED Reserved for future use.

RENEWABLE-OK The RENEWABLE-OK option indicates that a renewable ticket will be acceptable if a ticket with the requested life cannot otherwise be provided. If a ticket with the requested life cannot be provided, then a renewable ticket may be issued with a renew-till equal to the the requested endtime. The value of the renew-till field may still be limited by local limits, or limits selected by the individual principal or server.

ENC-TKT-IN-SKEY This option is used only by the ticket-granting service. The ENCTKT-IN-SKEY option indicates that the ticket for the end server is to be 


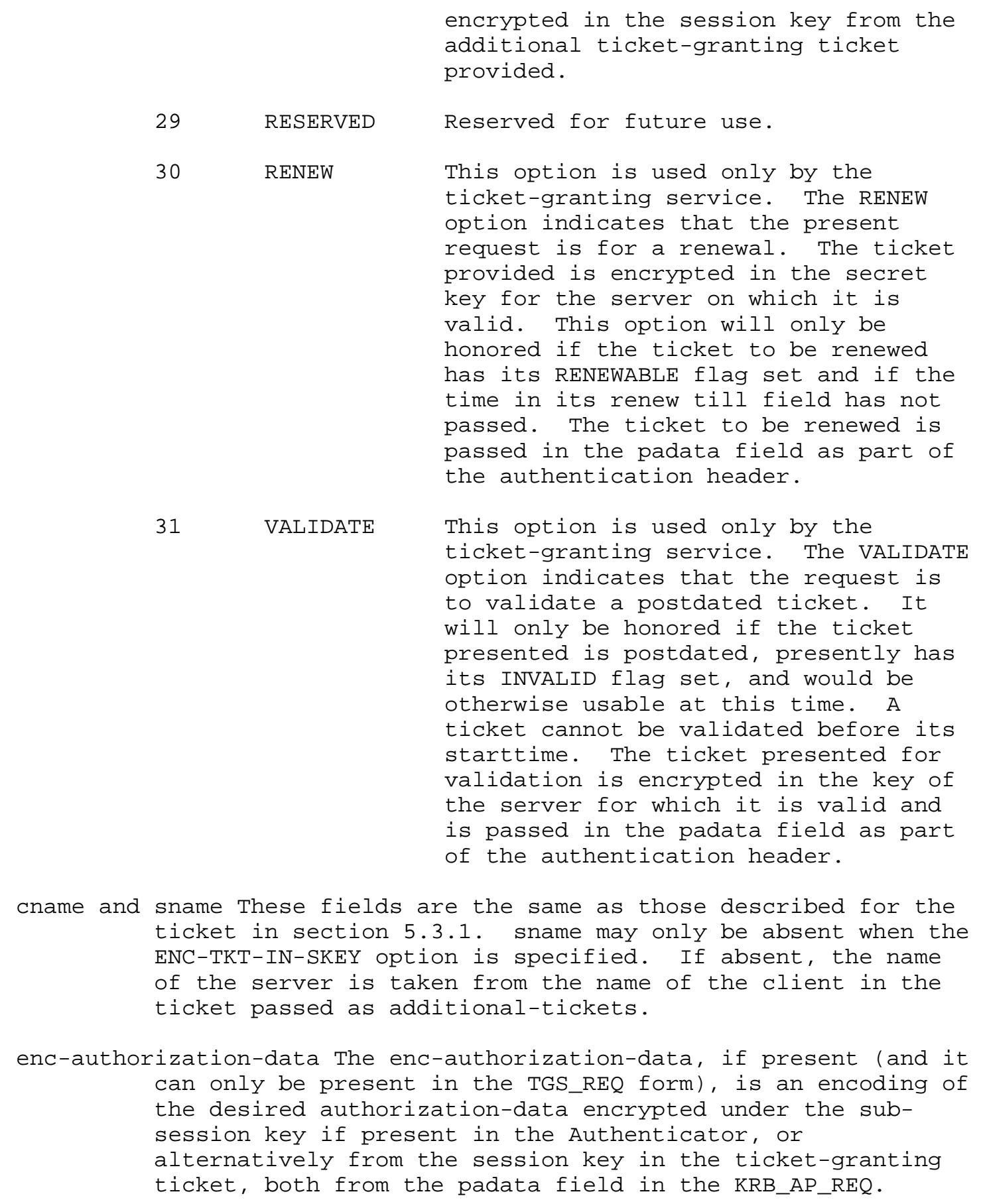




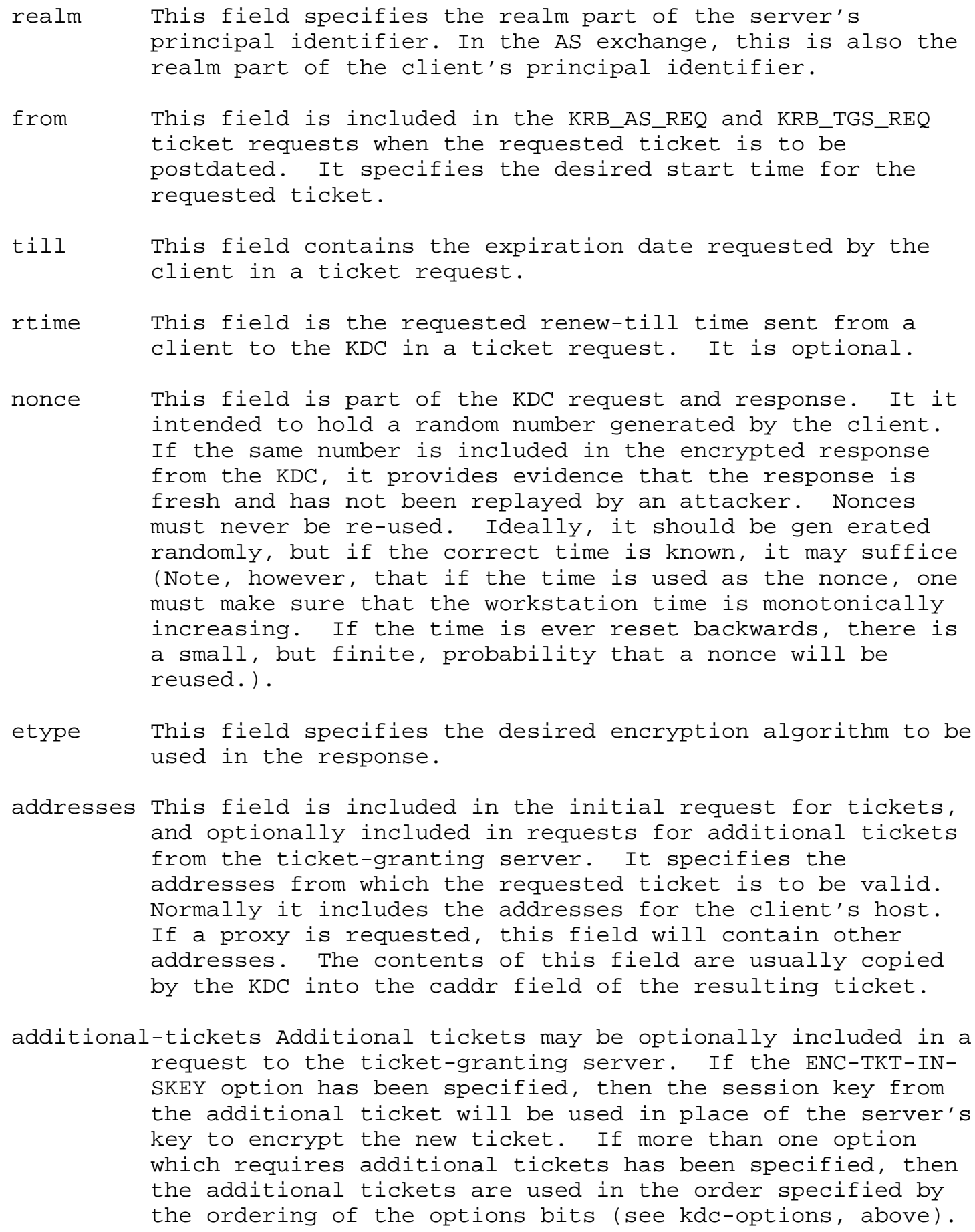


The application code will be either ten (10) or twelve (12) depending on whether the request is for an initial ticket ( $A S-R E Q$ ) or for an additional ticket (TGS-REQ).

The optional fields (addresses, authorization-data and additionaltickets) are only included if necessary to perform the operation specified in the kdc-options field.

It should be noted that in KRB_TGS_REQ, the protocol version number appears twice and two different message types appear: the KRB_TGS_REQ message contains these fields as does the authentication header (KRB_AP_REQ) that is passed in the padata field.

5.4.2. KRB_KDC_REP definition

The KRB_KDC_REP message format is used for the reply from the KDC for either an initial (AS) request or a subsequent (TGS) request. There is no message type for KRB_KDC_REP. Instead, the type will be either KRB_AS_REP or KRB_TGS_REP. The key used to encrypt the ciphertext part of the reply depends on the message type. For KRB_AS_REP, the ciphertext is encrypted in the client's secret key, and the client's key version number is included in the key version number for the encrypted data. For KRB_TGS_REP, the ciphertext is encrypted in the sub-session key from the Authenticator, or if absent, the session key from the ticket-granting ticket used in the request. In that case, no version number will be present in the EncryptedData sequence.

The KRB_KDC_REP message contains the following fields:

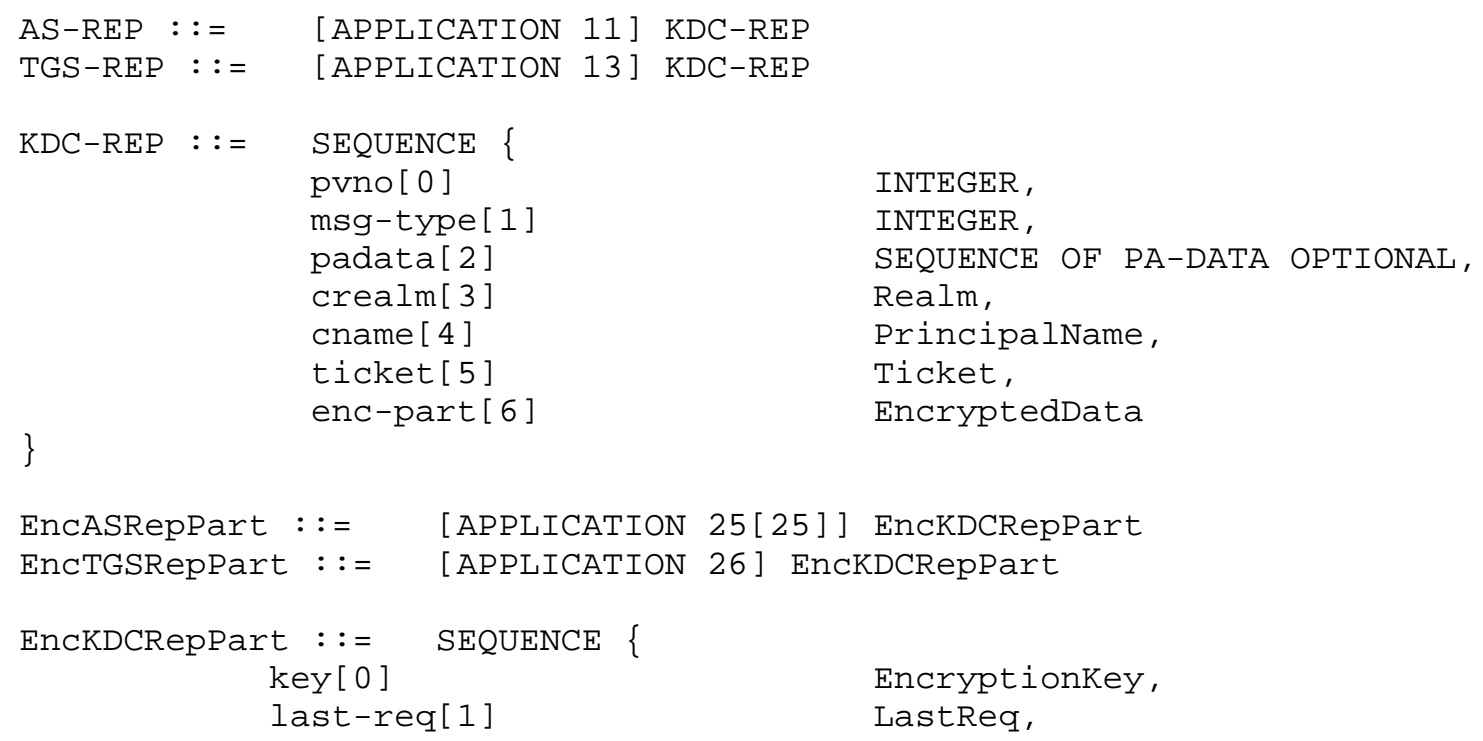




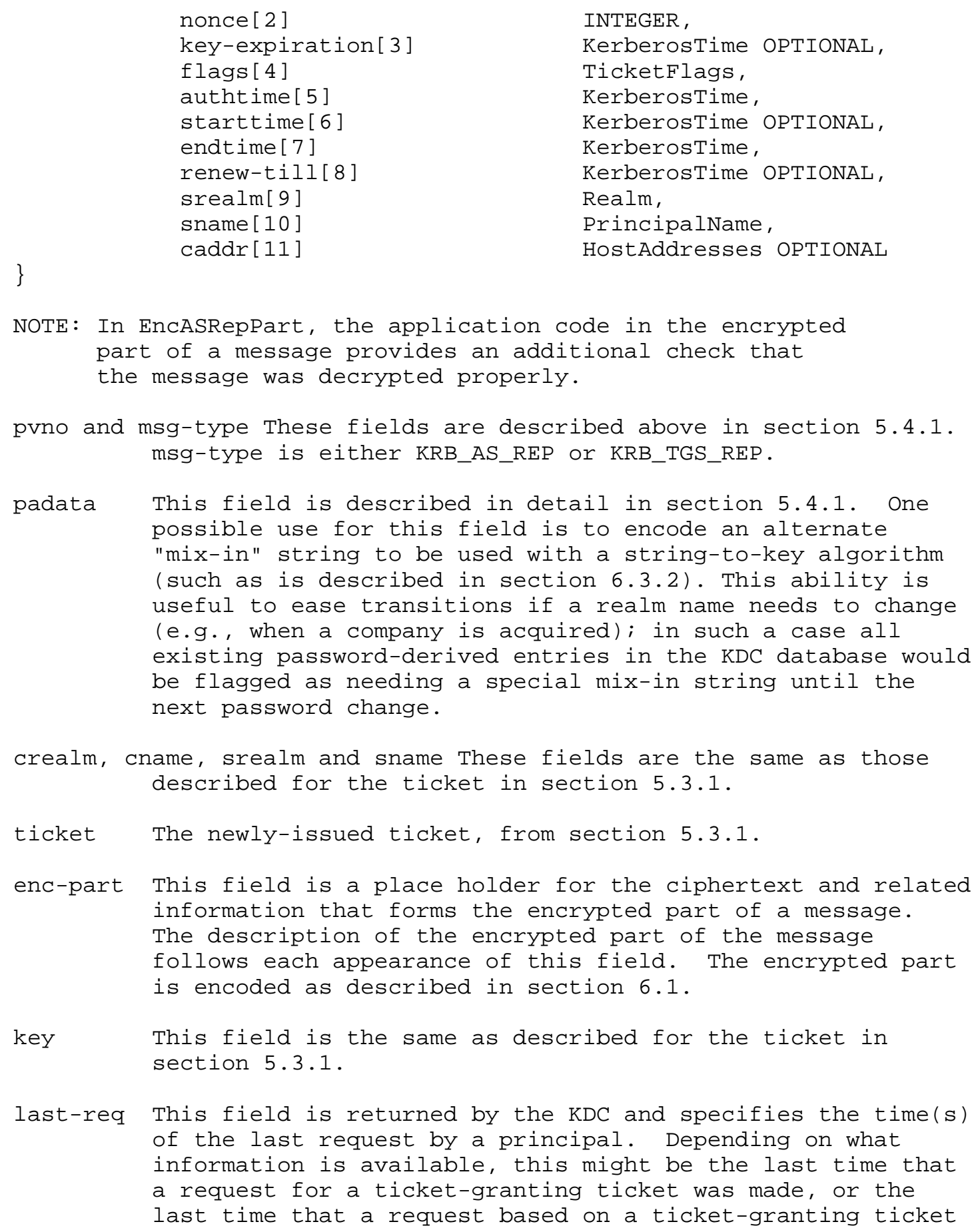

INTEGER,

Kerberostime OPTIONAL, TicketFlags, Kerberostime, Kerberostime OPTIONAL, Kerberostime, Kerberostime OPTIONAL, Realm, PrincipalName, HostAddresses OPTIONAL

NOTE: In EncASRepPart, the application code in the encrypted part of a message provides an additional check that the message was decrypted properly.

pvno and msg-type These fields are described above in section 5.4.1. msg-type is either KRB_AS_REP or KRB_TGS_REP.

padata This field is described in detail in section 5.4.1. One possible use for this field is to encode an alternate "mix-in" string to be used with a string-to-key algorithm (such as is described in section 6.3.2). This ability is useful to ease transitions if a realm name needs to change (e.g., when a company is acquired); in such a case all existing password-derived entries in the KDC database would be flagged as needing a special mix-in string until the next password change.

crealm, cname, srealm and sname These fields are the same as those described for the ticket in section 5.3.1.

ticket The newly-issued ticket, from section 5.3.1.

enc-part This field is a place holder for the ciphertext and related information that forms the encrypted part of a message. The description of the encrypted part of the message follows each appearance of this field. The encrypted part is encoded as described in section 6.1 .

key This field is the same as described for the ticket in section 5.3 .1 .

last-req This field is returned by the KDC and specifies the time(s) of the last request by a principal. Depending on what information is available, this might be the last time that a request for a ticket-granting ticket was made, or the last time that a request based on a ticket-granting ticket 
was successful. It also might cover all servers for a realm, or just the particular server. Some implementations may display this information to the user to aid in discovering unauthorized use of one's identity. It is similar in spirit to the last login time displayed when logging into timesharing systems.

nonce This field is described above in section 5.4.1.

key-expiration The key-expiration field is part of the response from the KDC and specifies the time that the client's secret key is due to expire. The expiration might be the result of password aging or an account expiration. This field will usually be left out of the TGS reply since the response to the TGS request is encrypted in a session key and no client information need be retrieved from the KDC database. It is up to the application client (usually the login program) to take appropriate action (such as notifying the user) if the expira tion time is imminent.

flags, authtime, starttime, endtime, renew-till and caddr These fields are duplicates of those found in the encrypted portion of the attached ticket (see section 5.3.1), provided so the client may verify they match the intended request and to assist in proper ticket caching. If the message is of type KRB_TGS_REP, the caddr field will only be filled in if the request was for a proxy or forwarded ticket, or if the user is substituting a subset of the addresses from the ticket granting ticket. If the clientrequested addresses are not present or not used, then the addresses contained in the ticket will be the same as those included in the ticket-granting ticket.

\subsection{Client/Server (CS) message specifications}

This section specifies the format of the messages used for the authentication of the client to the application server.

5.5.1. KRB_AP_REQ definition

The KRB_AP_REQ message contains the Kerberos protocol version number, the message type KRB_AP_REQ, an options field to indicate any options in use, and the ticket and authenticator themselves. The KRB_AP_REQ message is often referred to as the "authentication header".

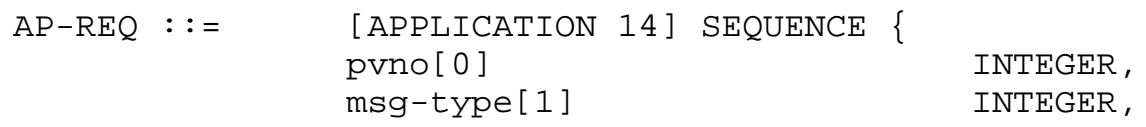




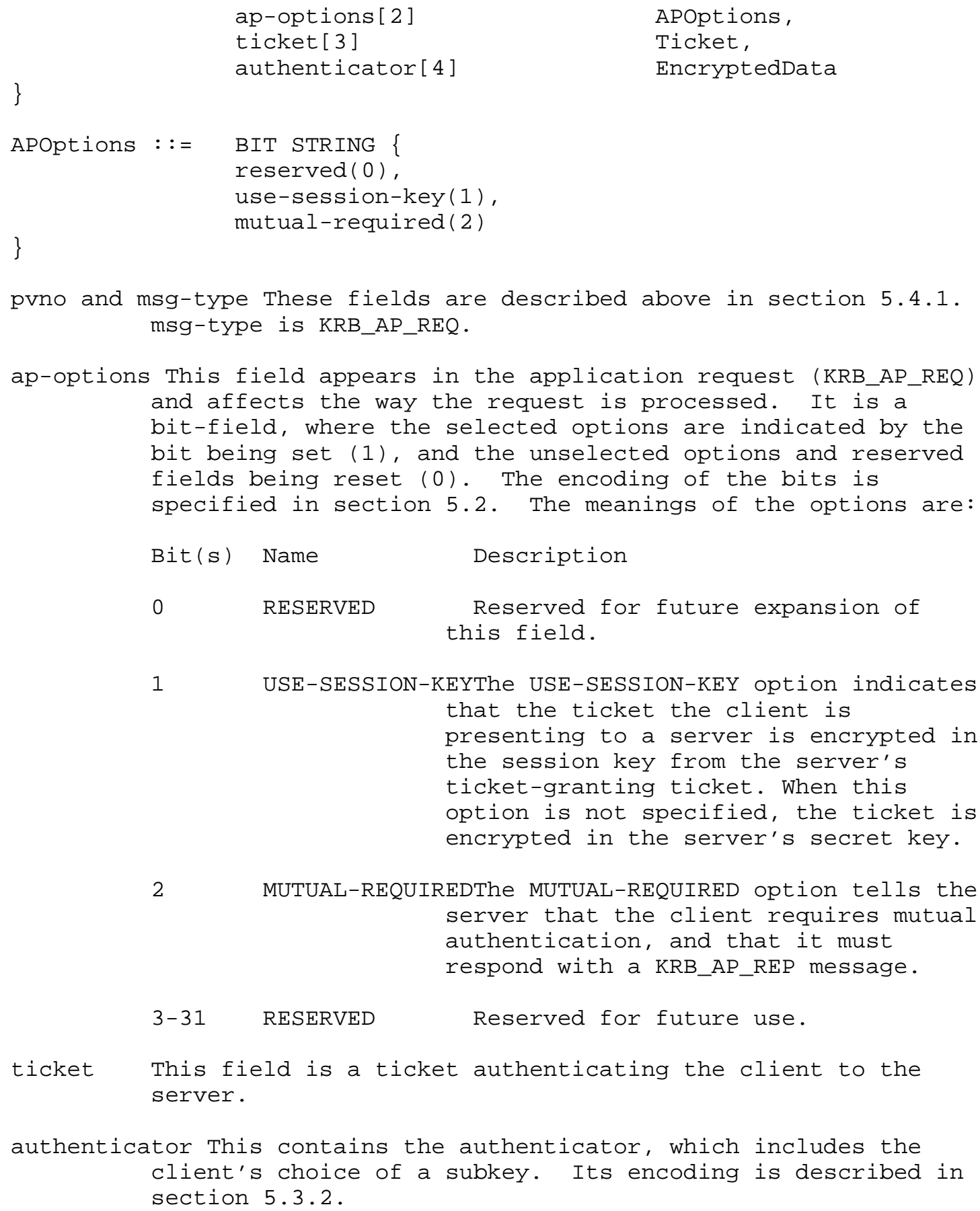




\subsubsection{KRB_AP_REP definition}

The KRB_AP_REP message contains the Kerberos protocol version number, the message type, and an encrypted timestamp. The message is sent in in response to an application request ( $K R B$ _AP_REQ) where the mutual authentication option has been selected in the ap-options field.

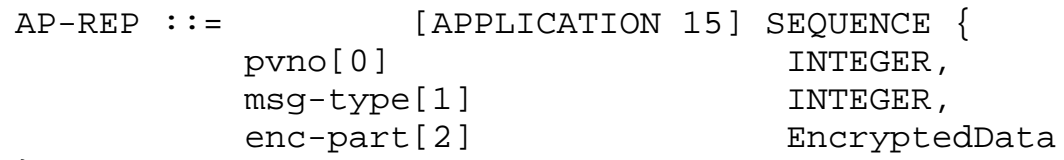




\subsubsection{Error message reply}

If an error occurs while processing the application request, the KRB_ERROR message will be sent in response. See section 5.9.1 for the format of the error message. The cname and crealm fields may be left out if the server cannot determine their appropriate values from the corresponding KRB_AP_REQ message. If the authenticator was decipherable, the ctime and cusec fields will contain the values from it.

5.6. KRB_SAFE message specification

This section specifies the format of a message that can be used by either side (client or server) of an application to send a tamperproof message to its peer. It presumes that a session key has previously been exchanged (for example, by using the KRB_AP_REQ/KRB_AP_REP messages).

5.6.1. KRB_SAFE definition

The KRB_SAFE message contains user data along with a collision-proof checksum keyed with the session key. The message fields are:

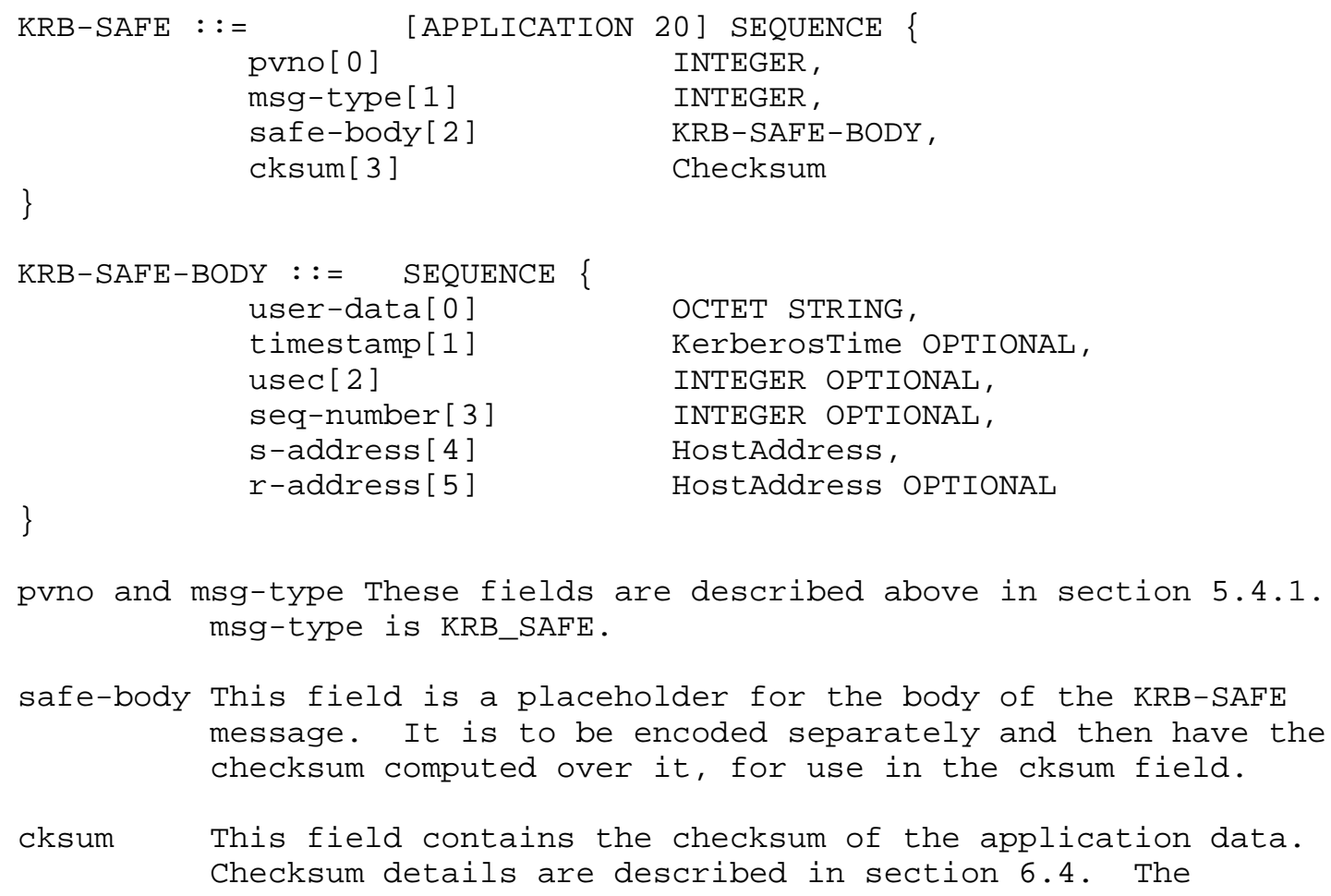


checksum is computed over the encoding of the KRB-SAFE-BODY sequence.

user-data This field is part of the KRB_SAFE and KRB_PRIV messages and contain the application specific data that is being passed from the sender to the recipient.

timestamp This field is part of the KRB_SAFE and KRB_PRIV messages. Its contents are the current time as known by the sender of the message. By checking the timestamp, the recipient of the message is able to make sure that it was recently generated, and is not a replay.

usec This field is part of the KRB_SAFE and KRB_PRIV headers. It contains the microsecond part of the timestamp.

seq-number This field is described above in section 5.3.2.

s-address This field specifies the address in use by the sender of the message.

r-address This field specifies the address in use by the recipient of the message. It may be omitted for some uses (such as broadcast protocols), but the recipient may arbitrarily reject such messages. This field along with s-address can be used to help detect messages which have been incorrectly or maliciously delivered to the wrong recipient.

5.7. KRB_PRIV message specification

This section specifies the format of a message that can be used by either side (client or server) of an application to securely and privately send a message to its peer. It presumes that a session key has previously been exchanged (for example, by using the

KRB_AP_REQ/KRB_AP_REP messages).

5.7.1. KRB_PRIV definition

The KRB_PRIV message contains user data encrypted in the Session Key. The message fields are:

$\begin{array}{rlr}\text { KRB-PRIV : }:= & \text { [APPLICATION 21] } & \text { SEQUENCE }\{ \\ & \text { pvno[0] } & \text { INTEGER, } \\ & \text { msg-type [1] } & \text { INTEGER, } \\ & \text { enc-part [3] } & \text { EncryptedData }\end{array}$




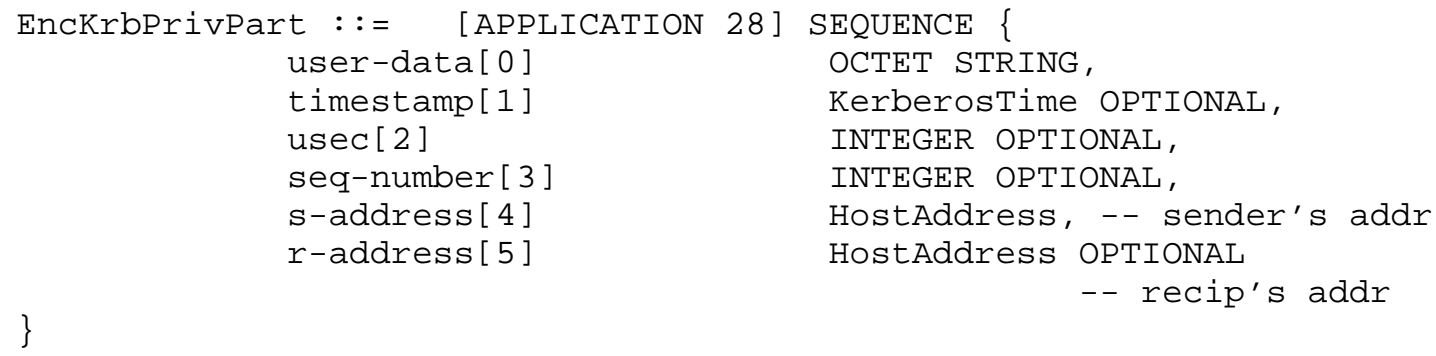


each. The information needed to use the tickets is encryped under an encryption key previously exchanged. The message fields are:

\begin{tabular}{|c|c|c|}
\hline $\mathrm{KRB}-\mathrm{CRED}$ & $\begin{array}{l}::=[\text { APPLICATION 22] } \\
\text { pvno[0] } \\
\text { msg-type [1] } \\
\text { tickets [2] } \\
\text { enc-part [3] }\end{array}$ & $\begin{array}{l}\text { SEQUENCE \{ } \\
\text { INTEGER, } \\
\text { INTEGER, -- KRB_CRED } \\
\text { SEQUENCE OF Ticket, } \\
\text { EncryptedData }\end{array}$ \\
\hline$\}$ & & \\
\hline EncKrbCredPart & $\begin{array}{l}::=[\text { APPLICATION 29] } \\
\text { ticket-info[0] } \\
\text { nonce [1] } \\
\text { timestamp [2] } \\
\text { usec[3] } \\
\text { s-address [4] } \\
\text { r-address [5] }\end{array}$ & $\begin{array}{l}\text { SEQUENCE \{ } \\
\text { SEQUENCE OF KrbCredInfo, } \\
\text { INTEGER OPTIONAL, } \\
\text { KerberOSTIme OPTIONAL, } \\
\text { INTEGER OPTIONAL, } \\
\text { HostAddresS OPTIONAL, } \\
\text { HostAddresS OPTIONAL }\end{array}$ \\
\hline$\}$ & & \\
\hline KrbCredInfo & $\begin{array}{l}::= \\
\text { key [0] } \\
\text { prealm [1] } \\
\text { pname [2] } \\
\text { flags [3] } \\
\text { authtime [4] } \\
\text { starttime [5] } \\
\text { endtime [6] } \\
\text { renew-till [7] } \\
\text { srealm [8] } \\
\text { sname [9] } \\
\text { caddr [10] }\end{array}$ & $\begin{array}{l}\text { SEQUENCE } \\
\text { EncryptionKey, } \\
\text { Realm OPTIONAL, } \\
\text { PrincipalName OPTIONAL, } \\
\text { TicketFlags OPTIONAL, } \\
\text { KerberosTime OPTIONAL, } \\
\text { KerberosTime OPTIONAL, } \\
\text { KerberosTime OPTIONAL } \\
\text { KerberosTime OPTIONAL, } \\
\text { Realm OPTIONAL, } \\
\text { PrincipalName OPTIONAL, } \\
\text { HostAddresses OPTIONAL }\end{array}$ \\
\hline
\end{tabular}

pvno and msg-type These fields are described above in section 5.4.1. msg-type is KRB_CRED.

tickets

These are the tickets obtained from the KDC specifically for use by the intended recipient. Successive tickets are paired with the corresponding KrbCredInfo sequence from the enc-part of the KRB-CRED message.

enc-part This field holds an encoding of the EncKrbCredPart sequence encrypted under the session key shared between the sender and the intended recipient. This encrypted encoding is used for the enc-part field of the KRB-CRED message. See section 6 for the format of the ciphertext. 
nonce If practical, an application may require the inclusion of a nonce generated by the recipient of the message. If the same value is included as the nonce in the message, it provides evidence that the message is fresh and has not been replayed by an attacker. A nonce must never be reused; it should be generated randomly by the recipient of the message and provided to the sender of the mes sage in an application specific manner.

timestamp and usec These fields specify the time that the KRB-CRED message was generated. The time is used to provide assurance that the message is fresh.

s-address and r-address These fields are described above in section 5.6.1. They are used optionally to provide additional assurance of the integrity of the KRB-CRED message.

key This field exists in the corresponding ticket passed by the KRB-CRED message and is used to pass the session key from the sender to the intended recipient. The field's encoding is described in section 6.2 .

The following fields are optional. If present, they can be associated with the credentials in the remote ticket file. If left out, then it is assumed that the recipient of the credentials already knows their value.

prealm and pname The name and realm of the delegated principal identity.

flags, authtime, starttime, endtime, renew-till, srealm, sname, and caddr These fields contain the values of the corresponding fields from the ticket found in the ticket field. Descriptions of the fields are identical to the descriptions in the $\mathrm{KDC}-\mathrm{REP}$ message.

\subsection{Error message specification}

This section specifies the format for the KRB_ERROR message. The fields included in the message are intended to return as much information as possible about an error. It is not expected that all the information required by the fields will be available for all types of errors. If the appropriate information is not available when the message is composed, the corresponding field will be left out of the message.

Note that since the KRB_ERROR message is not protected by any encryption, it is quite possible for an intruder to synthesize or 
modify such a message. In particular, this means that the client should not use any fields in this message for security-critical purposes, such as setting a system clock or generating a fresh authenticator. The message can be useful, however, for advising a user on the reason for some failure.

5.9.1. KRB_ERROR definition

The KRB_ERROR message consists of the following fields:

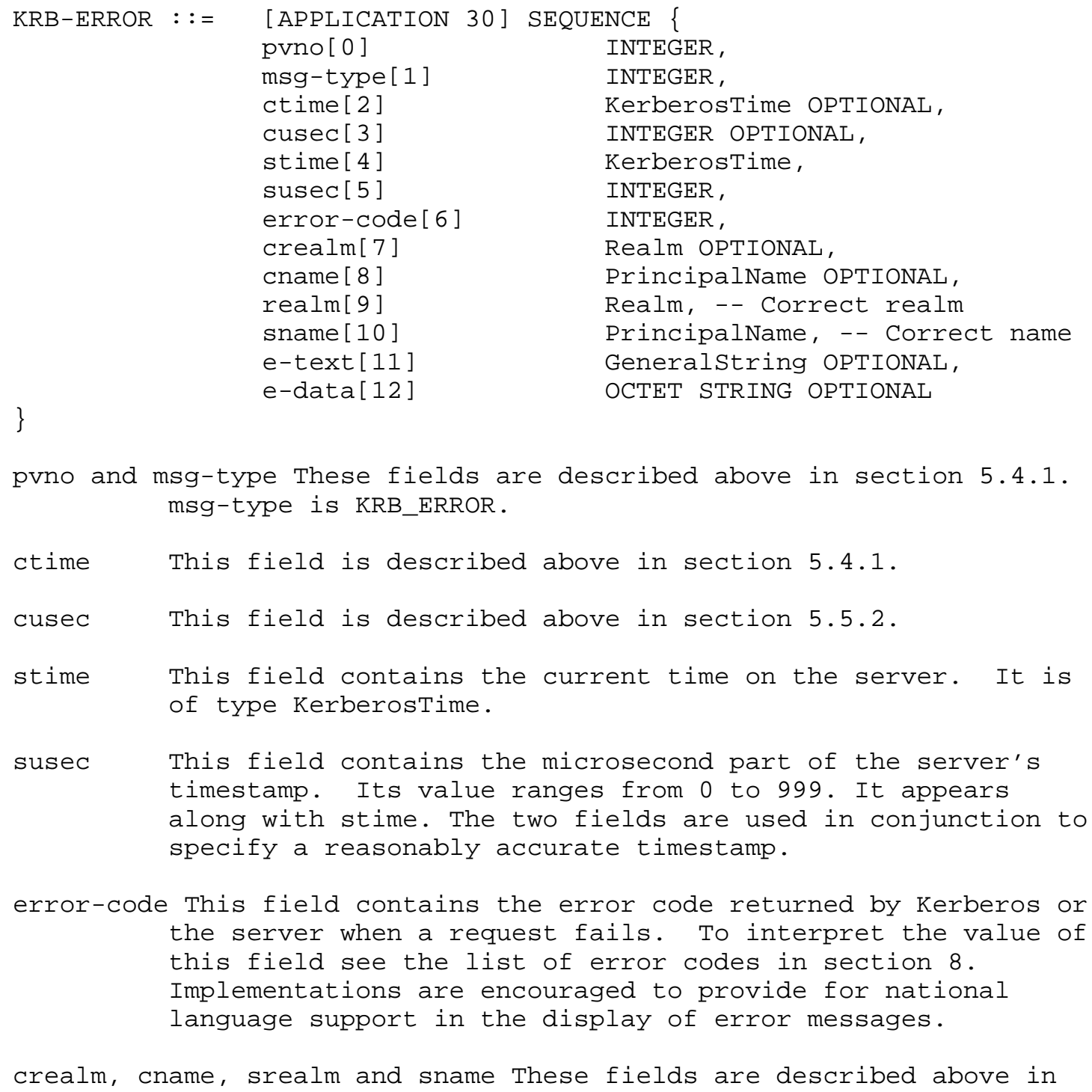


section 5.3 .1

e-text This field contains additional text to help explain the error code associated with the failed request (for example, it might include a principal name which was unknown).

e-data This field contains additional data about the error for use by the application to help it recover from or handle the error. If the errorcode is KDC_ERR_PREAUTH_REQUIRED, then the e-data field will contain an encoding of a sequence of padata fields, each corresponding to an acceptable preauthentication method and optionally containing data for the method:

METHOD-DATA : := SEQUENCE of PA-DATA

If the error-code is KRB_AP_ERR_METHOD, then the e-data field will contain an encoding of the following sequence:

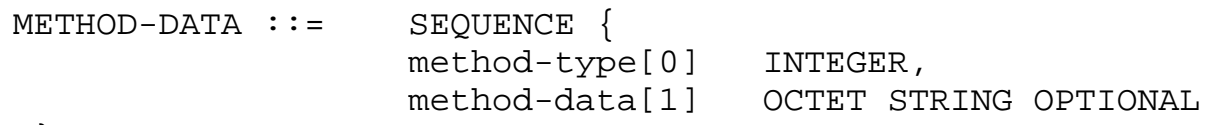

method-type will indicate the required alternate method; method-data will contain any required additional information.

6. Encryption and Checksum Specifications

The Kerberos protocols described in this document are designed to use stream encryption ciphers, which can be simulated using commonly available block encryption ciphers, such as the Data Encryption Standard [11], in conjunction with block chaining and checksum methods [12]. Encryption is used to prove the identities of the network entities participating in message exchanges. The Key Distribution Center for each realm is trusted by all principals registered in that realm to store a secret key in confidence. Proof of knowledge of this secret key is used to verify the authenticity of a principal.

The KDC uses the principal's secret key (in the AS exchange) or a shared session key (in the TGS exchange) to encrypt responses to ticket requests; the ability to obtain the secret key or session key implies the knowledge of the appropriate keys and the identity of the KDC. The ability of a principal to decrypt the KDC response and present a Ticket and a properly formed Authenticator (generated with the session key from the KDC response) to a service verifies the identity of the principal; likewise the ability of the service to 
extract the session key from the Ticket and prove its knowledge thereof in a response verifies the identity of the service.

The Kerberos protocols generally assume that the encryption used is secure from cryptanalysis; however, in some cases, the order of fields in the encrypted portions of messages are arranged to minimize the effects of poorly chosen keys. It is still important to choose good keys. If keys are derived from user-typed passwords, those passwords need to be well chosen to make brute force attacks more difficult. Poorly chosen keys still make easy targets for intruders.

The following sections specify the encryption and checksum mechanisms currently defined for Kerberos. The encodings, chaining, and padding requirements for each are described. For encryption methods, it is often desirable to place random information (often referred to as a confounder) at the start of the message. The requirements for a confounder are specified with each encryption mechanism.

Some encryption systems use a block-chaining method to improve the the security characteristics of the ciphertext. However, these chaining methods often don't provide an integrity check upon decryption. Such systems (such as DES in CBC mode) must be augmented with a checksum of the plaintext which can be verified at decryption and used to detect any tampering or damage. Such checksums should be good at detecting burst errors in the input. If any damage is detected, the decryption routine is expected to return an error indicating the failure of an integrity check. Each encryption type is expected to provide and verify an appropriate checksum. The specification of each encryption method sets out its checksum requirements.

Finally, where a key is to be derived from a user's password, an algorithm for converting the password to a key of the appropriate type is included. It is desirable for the string to key function to be one-way, and for the mapping to be different in different realms. This is important because users who are registered in more than one realm will often use the same password in each, and it is desirable that an attacker compromising the Kerberos server in one realm not obtain or derive the user's key in another.

For a discussion of the integrity characteristics of the candidate encryption and checksum methods considered for Kerberos, the the reader is referred to [13].

\subsection{Encryption Specifications}

The following ASN.1 definition describes all encrypted messages. The enc-part field which appears in the unencrypted part of messages in 


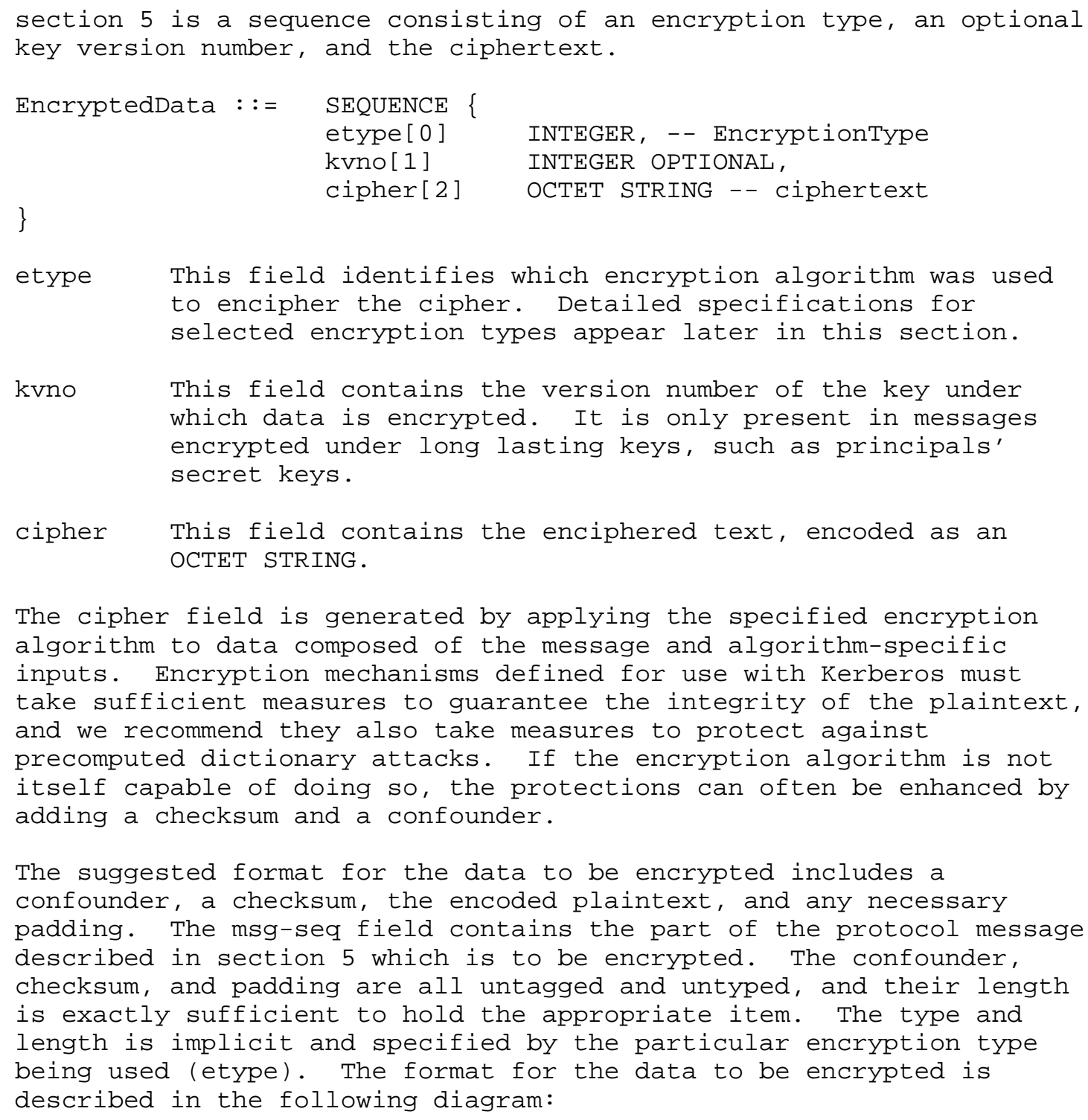

The cipher field is generated by applying the specified encryption algorithm to data composed of the message and algorithm-specific inputs. Encryption mechanisms defined for use with Kerberos must take sufficient measures to guarantee the integrity of the plaintext, and we recommend they also take measures to protect against precomputed dictionary attacks. If the encryption algorithm is not itself capable of doing so, the protections can often be enhanced by adding a checksum and a confounder.

The suggested format for the data to be encrypted includes a confounder, a checksum, the encoded plaintext, and any necessary padding. The msg-seq field contains the part of the protocol message described in section 5 which is to be encrypted. The confounder, checksum, and padding are all untagged and untyped, and their length is exactly sufficient to hold the appropriate item. The type and length is implicit and specified by the particular encryption type being used (etype). The format for the data to be encrypted is described in the following diagram:

The format cannot be described in ASN.1, but for those who prefer an ASN.1-like notation: 


\begin{tabular}{|c|c|c|c|}
\hline \multicolumn{4}{|c|}{ CipherText $::=\quad$ ENCRYPTED } \\
\hline confounder [0] & UNTAGGED OCTET & STRING (conf_length) & OPTIONAL, \\
\hline check [1] & UNTAGGED OCTET & STRING (checksum_length) & OPTIONAL, \\
\hline $\mathrm{msg}-\mathrm{seq}[2]$ & MsgSequence, & & \\
\hline pad & UNTAGGED OCTET & STRING (pad_length) OPTI & ONAL \\
\hline
\end{tabular}

In the above specification, UNTAGGED OCTET STRING(length) is the notation for an octet string with its tag and length removed. It is not a valid ASN.1 type. The tag bits and length must be removed from the confounder since the purpose of the confounder is so that the message starts with random data, but the tag and its length are fixed. For other fields, the length and tag would be redundant if they were included because they are specified by the encryption type.

One generates a random confounder of the appropriate length, placing it in confounder; zeroes out check; calculates the appropriate checksum over confounder, check, and msg-seq, placing the result in check; adds the necessary padding; then encrypts using the specified encryption type and the appropriate key.

Unless otherwise specified, a definition of an encryption algorithm that specifies a checksum, a length for the confounder field, or an octet boundary for padding uses this ciphertext format (The ordering of the fields in the CipherText is important. Additionally, messages encoded in this format must include a length as part of the msg-seq field. This allows the recipient to verify that the message has not been truncated. Without a length, an attacker could use a chosen plaintext attack to generate a message which could be truncated, while leaving the checksum intact. Note that if the msg-seq is an encoding of an ASN.1 SEQUENCE or OCTET STRING, then the length is part of that encoding.). Those fields which are not specified will be omitted.

In the interest of allowing all implementations using a particular encryption type to communicate with all others using that type, the specification of an encryption type defines any checksum that is needed as part of the encryption process. If an alternative checksum is to be used, a new encryption type must be defined.

Some cryptosystems require additional information beyond the key and the data to be encrypted. For example, DES, when used in cipherblock-chaining mode, requires an initialization vector. If required, the description for each encryption type must specify the source of such additional information. 


\subsection{Encryption Keys}

The sequence below shows the encoding of an encryption key:

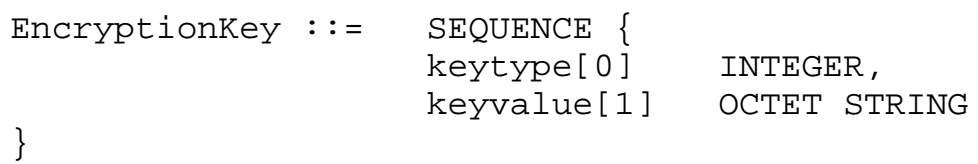

keytype This field specifies the type of encryption key that follows in the keyvalue field. It will almost always correspond to the encryption algorithm used to generate the EncryptedData, though more than one algorithm may use the same type of key (the mapping is many to one). This might happen, for example, if the encryption algorithm uses an alternate checksum algorithm for an integrity check, or a different chaining mechanism.

keyvalue This field contains the key itself, encoded as an octet string.

All negative values for the encryption key type are reserved for local use. All non-negative values are reserved for officially assigned type fields and interpretations.

\subsection{Encryption Systems}

6.3.1. The NULL Encryption System (null)

If no encryption is in use, the encryption system is said to be the NULL encryption system. In the NULL encryption system there is no checksum, confounder or padding. The ciphertext is simply the plaintext. The NULL Key is used by the null encryption system and is zero octets in length, with keytype zero (0).

6.3.2. DES in CBC mode with a CRC-32 checksum (des-cbc-crc)

The des-cbc-crc encryption mode encrypts information under the Data Encryption standard [11] using the cipher block chaining mode [12]. A CRC-32 checksum (described in ISO 3309 [14]) is applied to the confounder and message sequence (msg-seq) and placed in the cksum field. DES blocks are 8 bytes. As a result, the data to be encrypted (the concatenation of confounder, checksum, and message) must be padded to an 8 byte boundary before encryption. The details of the encryption of this data are identical to those for the descbc-ma5 encryption mode.

Note that, since the CRC-32 checksum is not collisionproof, an 
attacker could use a probabilistic chosenplaintext attack to generate a valid message even if a confounder is used [13]. The use of collision-proof checksums is recommended for environments where such attacks represent a significant threat. The use of the CRC-32 as the checksum for ticket or authenticator is no longer mandated as an interoperability requirement for Kerberos Version 5 specification 1 (See section 9.1 for specific details).

6.3.3. DES in CBC mode with an MD4 checksum (des-cbc-md4)

The des-cbc-md4 encryption mode encrypts information under the Data Encryption Standard [11] using the cipher block chaining mode [12]. An MD4 checksum (described in [15]) is applied to the confounder and message sequence (msg-seq) and placed in the cksum field. DES blocks are 8 bytes. As a result, the data to be encrypted (the concatenation of confounder, checksum, and message) must be padded to an 8 byte boundary before encryption. The details of the encryption of this data are identical to those for the descbc-md5 encryption mode.

6.3.4. DES in CBC mode with an MD5 checksum (des-cbc-md5)

The des-cbc-md5 encryption mode encrypts information under the Data Encryption Standard [11] using the cipher block chaining mode [12]. An MD5 checksum (described in [16]) is applied to the confounder and message sequence (msg-seq) and placed in the cksum field. DES blocks are 8 bytes. As a result, the data to be encrypted (the concatenation of confounder, checksum, and message) must be padded to an 8 byte boundary before encryption.

Plaintext and DES ciphtertext are encoded as 8-octet blocks which are concatenated to make the 64-bit inputs for the DES algorithms. The first octet supplies the 8 most significant bits (with the octet's MSbit used as the DES input block's MSbit, etc.), the second octet the next 8 bits, ..., and the eighth octet supplies the 8 least significant bits.

Encryption under DES using cipher block chaining requires an additional input in the form of an initialization vector. Unless otherwise specified, zero should be used as the initialization vector. Kerberos' use of DES requires an 8-octet confounder.

The DES specifications identify some "weak" and "semiweak" keys; those keys shall not be used for encrypting messages for use in Kerberos. Additionally, because of the way that keys are derived for the encryption of checksums, keys shall not be used that yield "weak" or "semi-weak" keys when exclusive-ORed with the constant FOFOFOFOFOFOFOFO. 
A DES key is 8 octets of data, with keytype one (1). This consists of 56 bits of key, and 8 parity bits (one per octet). The key is encoded as a series of 8 octets written in MSB-first order. The bits within the key are also encoded in MSB order. For example, if the encryption key is:

$(\mathrm{B} 1, \mathrm{~B} 2, \ldots, \mathrm{B} 7, \mathrm{P} 1, \mathrm{~B} 8, \ldots, \mathrm{B} 14, \mathrm{P} 2, \mathrm{~B} 15, \ldots, \mathrm{B} 49, \mathrm{P} 7, \mathrm{~B} 50, \ldots, \mathrm{B} 56, \mathrm{P} 8)$ where $\mathrm{B} 1, \mathrm{~B} 2, \ldots, \mathrm{B} 56$ are the key bits in MSB order, and P1,P2,..., P8 are the parity bits, the first octet of the key would be B1,B2,...,B7, P1 (with B1 as the MSbit). [See the FIPS 81 introduction for reference.]

To generate a DES key from a text string (password), the text string normally must have the realm and each component of the principal's name appended(In some cases, it may be necessary to use a different "mix-in" string for compatibility reasons; see the discussion of padata in section 5.4.2.), then padded with ASCII nulls to an 8 byte boundary. This string is then fan-folded and exclusive-oRed with itself to form an 8 byte DES key. The parity is corrected on the key, and it is used to generate a DES CBC checksum on the initial string (with the realm and name appended). Next, parity is corrected on the CBC checksum. If the result matches a "weak" or "semiweak" key as described in the DES specification, it is exclusive-ORed with the constant 00000000000000F0. Finally, the result is returned as the key. Pseudocode follows:

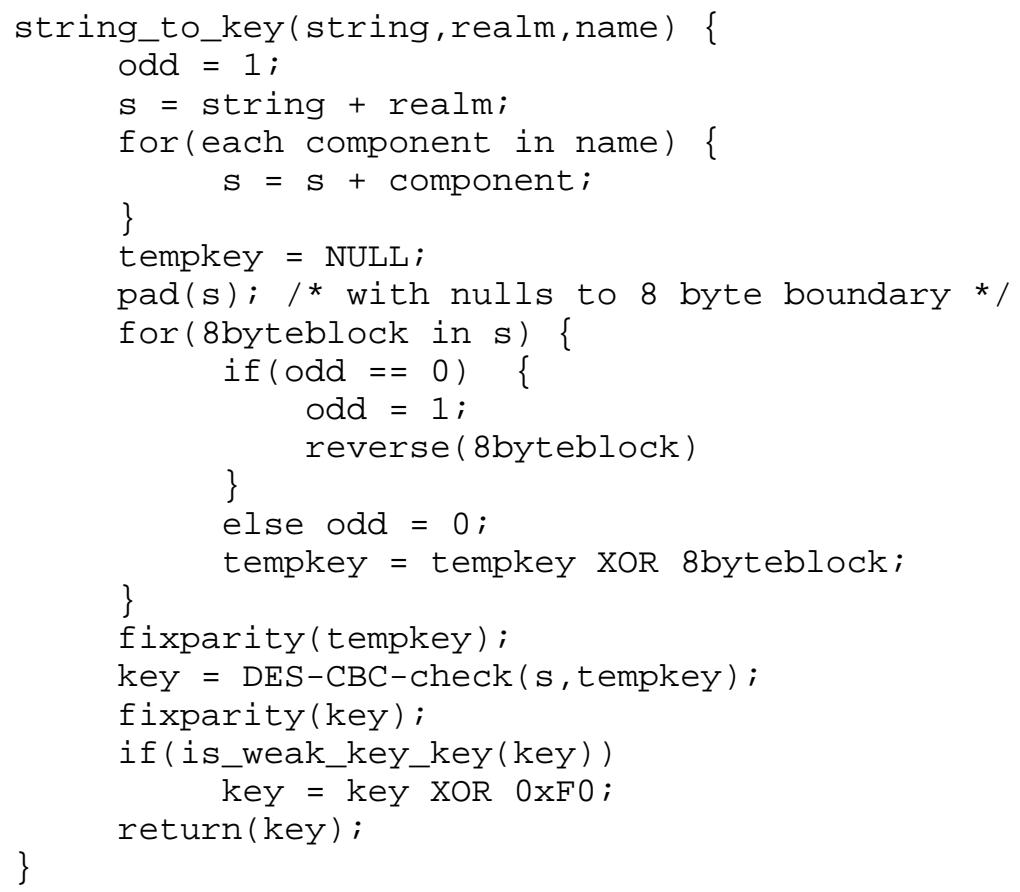


6.4. Checksums

The following is the ASN.1 definition used for a checksum:

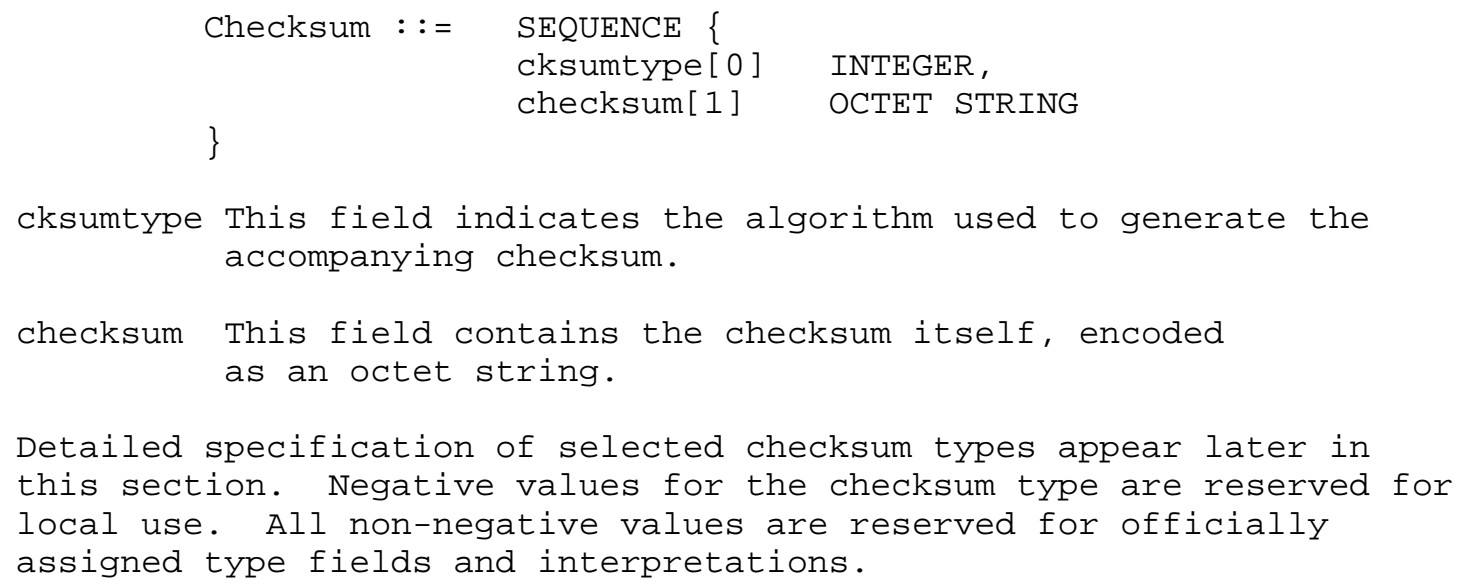

6.4.1. The CRC-32 Checksum (crc32)

The CRC-32 checksum calculates a checksum based on a cyclic redundancy check as described in ISO 3309 [14]. The resulting checksum is four (4) octets in length. The CRC-32 is neither keyed nor collision-proof. The use of this checksum is not recommended. An attacker using a probabilistic chosen-plaintext attack as described in [13] might be able to generate an alternative message that satisfies the checksum. The use of collision-proof checksums is recommended for environments where such attacks represent a 
significant threat.

\subsubsection{The RSA MD4 Checksum (rsa-md4)}

The RSA-MD4 checksum calculates a checksum using the RSA MD4 algorithm [15]. The algorithm takes as input an input message of arbitrary length and produces as output a 128-bit (16 octet) checksum. RSA-MD4 is believed to be collision-proof.

\subsubsection{RSA MD4 Cryptographic Checksum Using DES (rsa-md4des)}

The RSA-MD4-DES checksum calculates a keyed collisionproof checksum by prepending an 8 octet confounder before the text, applying the RSA MD 4 checksum algorithm, and encrypting the confounder and the checksum using DES in cipher-block-chaining (CBC) mode using a variant of the key, where the variant is computed by exclusive-oRing the key with the constant FOFOFOFOFOFOFOFO (A variant of the key is used to limit the use of a key to a particular function, separating the functions of generating a checksum from other encryption performed using the session key. The constant FOFOFOFOFOFOFOFO was chosen because it maintains key parity. The properties of DES precluded the use of the complement. The same constant is used for similar purpose in the Message Integrity Check in the Privacy Enhanced Mail standard.). The initialization vector should be zero. The resulting checksum is 24 octets long ( 8 octets of which are redundant). This checksum is tamper-proof and believed to be collision-proof.

The DES specifications identify some "weak keys"; those keys shall not be used for generating RSA-MD4 checksums for use in Kerberos.

The format for the checksum is described in the following diagram:

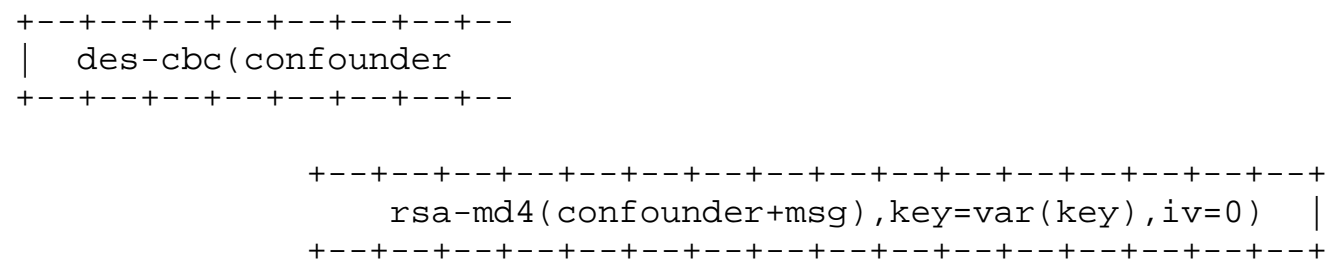

The format cannot be described in ASN.1, but for those who prefer an ASN.1-like notation:

$\begin{array}{lll}\text { rsa-md4-des-checksum : := } & \text { ENCRYPTED } & \text { UNTAGGED SEQUENCE }\{ \\ & \text { confounder [0] } & \text { UNTAGGED OCTET STRING (8), } \\ & \text { check[1] } & \text { UNTAGGED OCTET STRING (16) }\end{array}$


6.4.4. The RSA MD5 Checksum (rsa-md5)

The RSA-MD5 checksum calculates a checksum using the RSA MD5 algorithm [16]. The algorithm takes as input an input message of arbitrary length and produces as output a 128-bit (16 octet) checksum. RSA-MD5 is believed to be collision-proof.

6.4.5. RSA MD5 Cryptographic Checksum Using DES (rsa-md5des)

The RSA-MD5-DES checksum calculates a keyed collisionproof checksum by prepending an 8 octet confounder before the text, applying the RSA MD5 checksum algorithm, and encrypting the confounder and the checksum using DES in cipher-block-chaining (CBC) mode using a variant of the key, where the variant is computed by exclusive-oring the key with the constant FOFOFOFOFOFOFOFO. The initialization vector should be zero. The resulting checksum is 24 octets long ( 8 octets of which are redundant). This checksum is tamper-proof and believed to be collision-proof.

The DES specifications identify some "weak keys"; those keys shall not be used for encrypting RSA-MD5 checksums for use in Kerberos.

The format for the checksum is described in the following diagram:

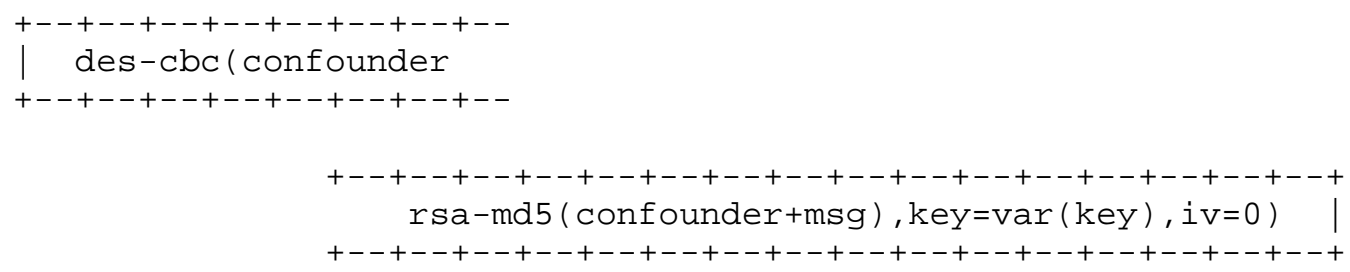

The format cannot be described in ASN.1, but for those who prefer an ASN.1-like notation:

rsa-md5-des-checksum : :=

ENCRYPTED

confounder [0]

check [1]
UNTAGGED SEQUENCE \{

UNTAGGED OCTET STRING(8), UNTAGGED OCTET STRING(16)

\}

6.4.6. DES cipher-block chained checksum (des-mac)

The DES-MAC checksum is computed by prepending an 8 octet confounder to the plaintext, performing a DES CBC-mode encryption on the result using the key and an initialization vector of zero, taking the last block of the ciphertext, prepending the same confounder and encrypting the pair using DES in cipher-block-chaining (CBC) mode using a a variant of the key, where the variant is computed by 
eXclusive-ORing the key with the constant FOFOFOFOFOFOFOFO. The initialization vector should be zero. The resulting checksum is 128 bits (16 octets) long, 64 bits of which are redundant. This checksum is tamper-proof and collision-proof.

The format for the checksum is described in the following diagram:

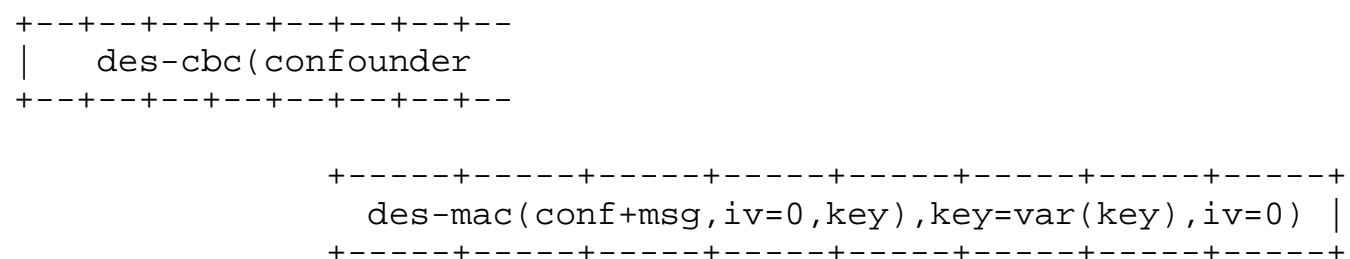

The DES specifications identify some "weak" and "semiweak" keys; those keys shall not be used for generating DES-MAC checksums for use in Kerberos, nor shall a key be used whose veriant is "weak" or "semi-weak".

6.4.7. RSA MD4 Cryptographic Checksum Using DES alternative (rsa-md4-des-k)

The RSA-MD4-DES-K checksum calculates a keyed collision-proof checksum by applying the RSA MD4 checksum algorithm and encrypting the results using DES in cipherblock-chaining ( $\mathrm{CBC}$ ) mode using a DES key as both key and initialization vector. The resulting checksum is 16 octets long. This checksum is tamper-proof and believed to be collision-proof. Note that this checksum type is the old method for encoding the RSA-MD4-DES checksum and it is no longer recommended.

6.4.8. DES cipher-block chained checksum alternative (desmac-k)

The DES-MAC-K checksum is computed by performing a DES CBC-mode encryption of the plaintext, and using the last block of the ciphertext as the checksum value. It is keyed with an encryption key and an initialization vector; any uses which do not specify an additional initialization vector will use the key as both key and initialization vector. The resulting checksum is 64 bits (8 octets) long. This checksum is tamper-proof and collision-proof. Note that 
this checksum type is the old method for encoding the DESMAC checksum and it is no longer recommended.

The DES specifications identify some "weak keys"; those keys shall not be used for generating DES-MAC checksums for use in Kerberos.

7. Naming Constraints

\subsection{Realm Names}

Although realm names are encoded as Generalstrings and although a realm can technically select any name it chooses, interoperability across realm boundaries requires agreement on how realm names are to be assigned, and what information they imply.

To enforce these conventions, each realm must conform to the conventions itself, and it must require that any realms with which inter-realm keys are shared also conform to the conventions and require the same from its neighbors.

There are presently four styles of realm names: domain, x500, other, and reserved. Examples of each style follow:

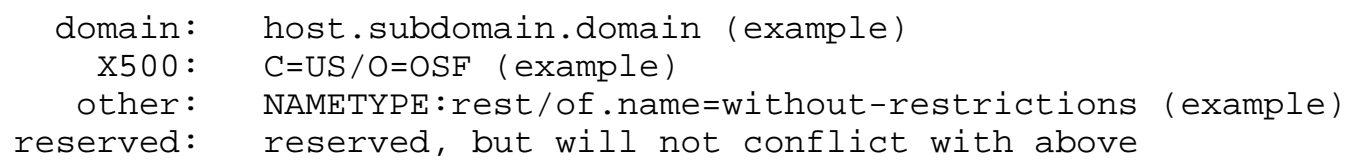


various name styles. The following additional constraints apply to the assignment of realm names in the domain and X.500 categories: the name of a realm for the domain or $\mathrm{x} .500$ formats must either be used by the organization owning (to whom it was assigned) an Internet domain name or X.500 name, or in the case that no such names are registered, authority to use a realm name may be derived from the authority of the parent realm. For example, if there is no domain name for E40.MIT.EDU, then the administrator of the MIT.EDU realm can authorize the creation of a realm with that name.

This is acceptable because the organization to which the parent is assigned is presumably the organization authorized to assign names to its children in the X.500 and domain name systems as well. If the parent assigns a realm name without also registering it in the domain name or $\mathrm{X} .500$ hierarchy, it is the parent's responsibility to make sure that there will not in the future exists a name identical to the realm name of the child unless it is assigned to the same entity as the realm name.

\subsection{Principal Names}

As was the case for realm names, conventions are needed to ensure that all agree on what information is implied by a principal name. The name-type field that is part of the principal name indicates the kind of information implied by the name. The name-type should be treated as a hint. Ignoring the name type, no two names can be the same (i.e., at least one of the components, or the realm, must be different). This constraint may be eliminated in the future. The following name types are defined:

\begin{tabular}{|c|c|c|}
\hline name-type & value & meaning \\
\hline NT-UNKNOWN & 0 & Name type not known \\
\hline NT-PRINCIPAL & 1 & $\begin{array}{l}\text { Just the name of the principal as in } \\
\text { DCE, or for users }\end{array}$ \\
\hline $\mathrm{NT}-\mathrm{SRV}-\mathrm{INST}$ & 2 & Service and other unique instance (krbtgt) \\
\hline $\mathrm{NT}-\mathrm{SRV}-\mathrm{HST}$ & 3 & $\begin{array}{l}\text { Service with host name as instance } \\
\text { (telnet, rcommands) }\end{array}$ \\
\hline $\mathrm{NT}-\mathrm{SRV}-\mathrm{XHST}$ & 4 & Service with host as remaining components \\
\hline $\mathrm{NT}-\mathrm{UID}$ & 5 & Unique ID \\
\hline
\end{tabular}

When a name implies no information other than its uniqueness at a particular time the name type PRINCIPAL should be used. The principal name type should be used for users, and it might also be used for a unique server. If the name is a unique machine generated ID that is guaranteed never to be reassigned then the name type of UID should be used (note that it is generally a bad idea to reassign names of any type since stale entries might remain in access control lists). 
If the first component of a name identifies a service and the remaining components identify an instance of the service in a server specified manner, then the name type of SRV-INST should be used. An example of this name type is the Kerberos ticket-granting ticket which has a first component of krbtgt and a second component identifying the realm for which the ticket is valid.

If instance is a single component following the service name and the instance identifies the host on which the server is running, then the name type SRV-HST should be used. This type is typically used for Internet services such as telnet and the Berkeley $R$ commands. If the separate components of the host name appear as successive components following the name of the service, then the name type SRVXHST should be used. This type might be used to identify servers on hosts with X.500 names where the slash (/) might otherwise be ambiguous.

A name type of UNKNOWN should be used when the form of the name is not known. When comparing names, a name of type UNKNOWN will match principals authenticated with names of any type. A principal authenticated with a name of type UNKNOWN, however, will only match other names of type UNKNOWN.

Names of any type with an initial component of "krbtgt" are reserved for the Kerberos ticket granting service. See section 8.2.3 for the form of such names.

7.2.1. Name of server principals

The principal identifier for a server on a host will generally be composed of two parts: (1) the realm of the KDC with which the server is registered, and (2) a two-component name of type NT-SRV-HST if the host name is an Internet domain name or a multi-component name of type NT-SRV-XHST if the name of the host is of a form such as X.500 that allows slash (/) separators. The first component of the two- or multi-component name will identify the service and the latter

components will identify the host. Where the name of the host is not case sensitive (for example, with Internet domain names) the name of the host must be lower case. For services such as telnet and the Berkeley $R$ commands which run with system privileges, the first component will be the string "host" instead of a service specific identifier.

\section{Constants and other defined values}

\subsection{Host address types}

All negative values for the host address type are reserved for local use. All non-negative values are reserved for officially assigned 
type fields and interpretations.

The values of the types for the following addresses are chosen to match the defined address family constants in the Berkeley Standard Distributions of Unix. They can be found in <sys/socket.h> with symbolic names $A F \_x x x$ (where $x x x$ is an abbreviation of the address family name).

Internet addresses

Internet addresses are 32-bit (4-octet) quantities, encoded in MSB order. The type of internet addresses is two (2).

CHAOSnet addresses

CHAOSnet addresses are 16-bit (2-octet) quantities, encoded in MSB order. The type of CHAOsnet addresses is five (5).

ISO addresses

ISO addresses are variable-length. The type of ISO addresses is seven (7).

Xerox Network Services (XNS) addresses

XNS addresses are 48-bit (6-octet) quantities, encoded in MSB order. The type of XNS addresses is six (6).

AppleTalk Datagram Delivery Protocol (DDP) addresses

AppleTalk DDP addresses consist of an 8-bit node number and a 16bit network number. The first octet of the address is the node number; the remaining two octets encode the network number in MSB order. The type of AppleTalk DDP addresses is sixteen (16).

DECnet Phase IV addresses

DECnet Phase IV addresses are 16-bit addresses, encoded in LSB

order. The type of DECnet Phase IV addresses is twelve (12).

8.2. KDC messages

8.2.1. IP transport

When contacting a Kerberos server (KDC) for a KRB_KDC_REQ request using IP transport, the client shall send a UDP datagram containing only an encoding of the request to port 88 (decimal) at the KDC's IP 
address; the KDC will respond with a reply datagram containing only an encoding of the reply message (either a KRB_ERROR or a KRB_KDC_REP) to the sending port at the sender's IP address.

\subsubsection{OSI transport}

During authentication of an OSI client to and OSI server, the mutual authentication of an OSI server to an OSI client, the transfer of credentials from an OSI client to an OSI server, or during exchange of private or integrity checked messages, Kerberos protocol messages may be treated as opaque objects and the type of the authentication mechanism will be:

OBJECT IDENTIFIER $::=\{$ iso (1), org(3), dod(5), internet (1), security(5), kerberosv5(2) \}

Depending on the situation, the opaque object will be an authentication header (KRB_AP_REQ), an authentication reply (KRB_AP_REP), a safe message (KRB_SAFE), a private message (KRB_PRIV), or a credentials message (KRB_CRED). The opaque data contains an application code as specified in the ASN.1 description for each message. The application code may be used by Kerberos to determine the message type.

8.2.3. Name of the TGS

The principal identifier of the ticket-granting service shall be composed of three parts: (1) the realm of the KDC issuing the TGS ticket (2) a two-part name of type NT-SRVINST, with the first part "krbtgt" and the second part the name of the realm which will accept the ticket-granting ticket. For example, a ticket-granting ticket issued by the ATHENA.MIT.EDU realm to be used to get tickets from the ATHENA.MIT.EDU KDC has a principal identifier of "ATHENA.MIT.EDU" (realm), ("krbtgt", "ATHENA.MIT.EDU") (name). A ticket-granting ticket issued by the ATHENA.MIT.EDU realm to be used to get tickets from the MIT.EDU realm has a principal identifier of "ATHENA.MIT.EDU" (realm), ("krbtgt", "MIT.EDU") (name).

\subsection{Protocol constants and associated values}

The following tables list constants used in the protocol and defines their meanings. 


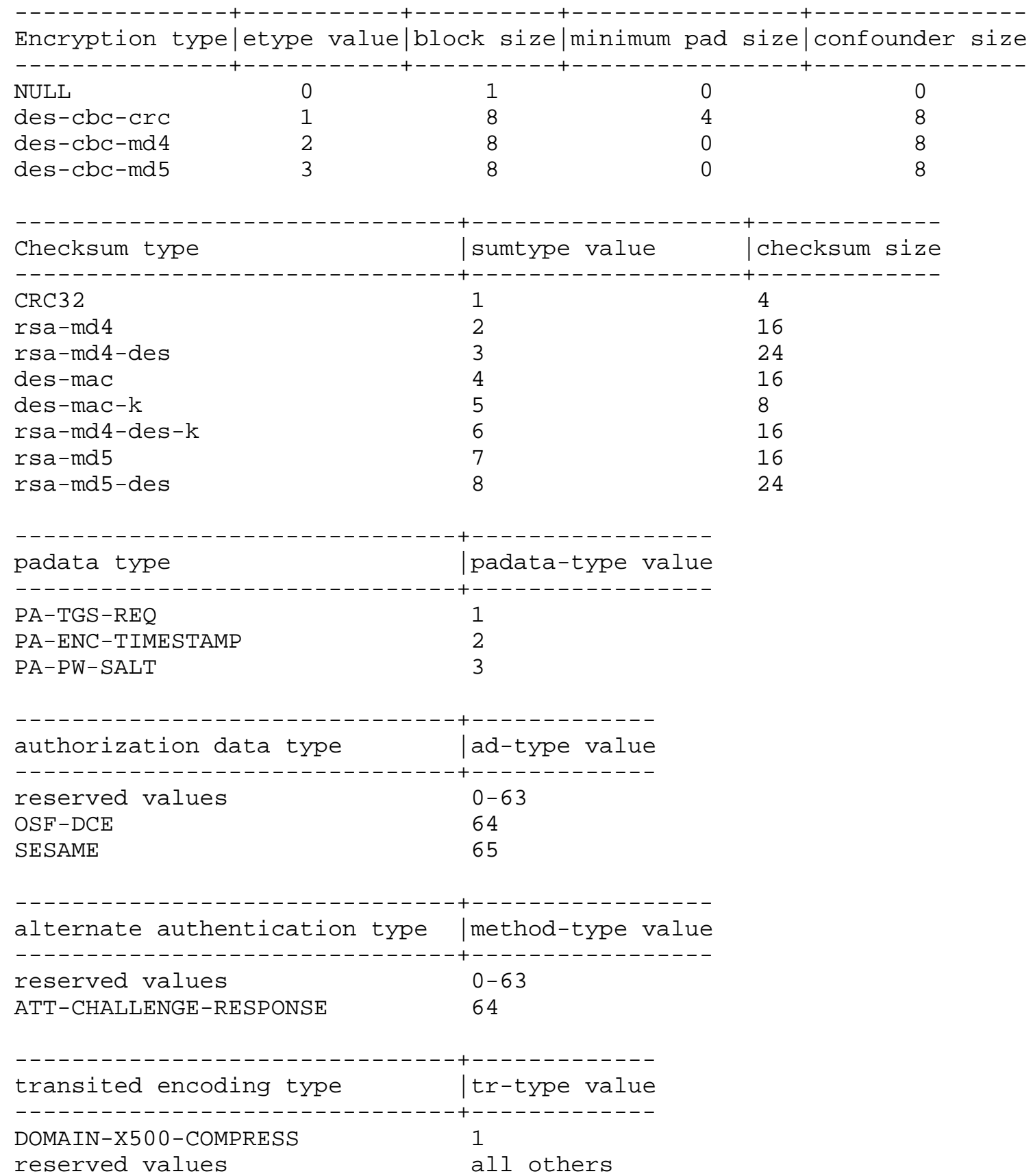




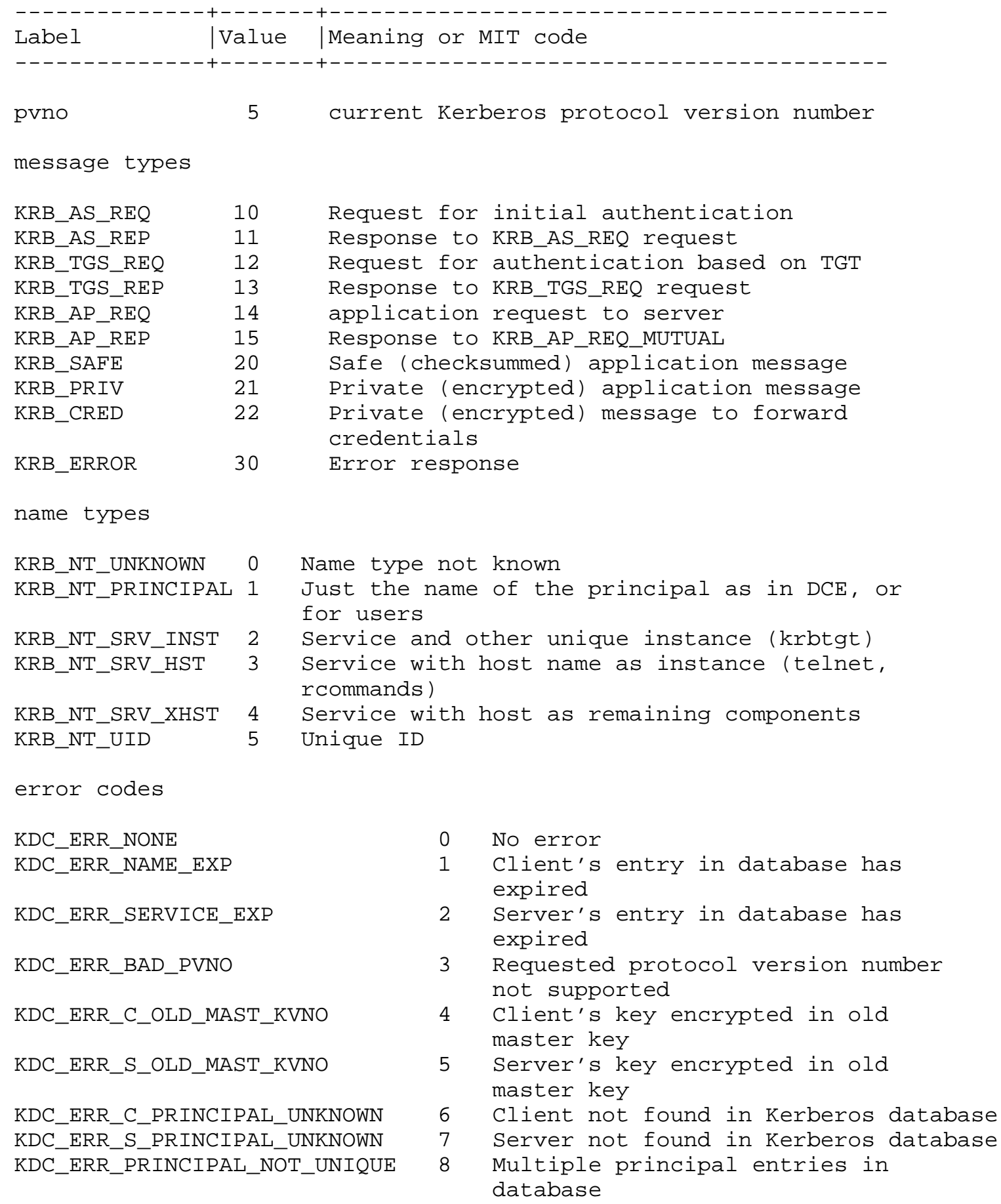


KDC ERR NULL KEY

KDC_ERR_CANNOT_POSTDATE

KDC_ERR_NEVER_VALID

KDC_ERR_POLICY

KDC_ERR_BADOPTION

KDC_ERR_ETYPE_NOSUPP

KDC_ERR_SUMTYPE_NOSUPP

KDC_ERR_PADATA_TYPE_NOSUPP

KDC_ERR_TRTYPE_NOSUPP

KDC_ERR_CLIENT_REVOKED

KDC_ERR_SERVICE_REVOKED

KDC_ERR_TGT_REVOKED

KDC_ERR_CLIENT_NOTYET

KDC_ERR_SERVICE_NOTYET

KDC_ERR_KEY_EXPIRED

KDC_ERR_PREAUTH_FAILED

KDC_ERR_PREAUTH_REQUIRED

KRB_AP_ERR_BAD_INTEGRITY

KRB_AP_ERR_TKT_EXPIRED

KRB_AP_ERR_TKT_NYV

KRB AP ERR REPEAT

KRB_AP_ERR_NOT_US

KRB_AP_ERR_BADMATCH

KRB_AP_ERR_SKEW

KRB_AP_ERR_BADADDR

KRB_AP_ERR_BADVERSION

KRB_AP_ERR_MSG_TYPE

KRB_AP_ERR_MODIFIED

KRB_AP_ERR_BADORDER

KRB_AP_ERR_BADKEYVER

KRB_AP_ERR_NOKEY

KRB_AP_ERR_MUT_FAIL

KRB_AP_ERR_BADDIRECTION

KRB_AP_ERR_METHOD

KRB_AP_ERR_BADSEQ

KRB_AP_ERR_INAPP_CKSUM
10
The client or server has a null key Ticket not eligible for postdating Requested start time is later than end time KDC policy rejects request KDC cannot accommodate requested option

KDC has no support for encryption type KDC has no support for checksum type KDC has no support for padata type $\mathrm{KDC}$ has no support for transited type Clients credentials have been revoked Credentials for server have been revoked

TGT has been revoked

client not yet valid - try again

later

Server not yet valid - try again

later

Password has expired - change

password to reset

Pre-authentication information

was invalid

Additional pre-authentication

required*

Integrity check on decrypted field

failed

Ticket expired

Ticket not yet valid

Request is a replay

The ticket isn't for us

Ticket and authenticator don't match

Clock skew too great

Incorrect net address

Protocol version mismatch

Invalid msg type

Message stream modified

Message out of order

Specified version of key is not available

Service key not available

Mutual authentication failed

Incorrect message direction

Alternative authentication method required*

Incorrect sequence number in message

Inappropriate type of checksum in 
KRB_ERR_GENERIC

KRB_ERR_FIELD_TOOLONG message

60 Generic error (description in e-text)

61 Field is too long for this

implementation

*This error carries additional information in the e-data field. The contents of the e-data field for this message is described in section 5.9 .1 .

9. Interoperability requirements

Version 5 of the Kerberos protocol supports a myriad of options. Among these are multiple encryption and checksum types, alternative encoding schemes for the transited field, optional mechanisms for pre-authentication, the handling of tickets with no addresses, options for mutual authentication, user to user authentication, support for proxies, forwarding, postdating, and renewing tickets, the format of realm names, and the handling of authorization data.

In order to ensure the interoperability of realms, it is necessary to define a minimal configuration which must be supported by all implementations. This minimal configuration is subject to change as technology does. For example, if at some later date it is discovered that one of the required encryption or checksum algorithms is not secure, it will be replaced.

\subsection{Specification 1}

This section defines the first specification of these options. Implementations which are configured in this way can be said to support Kerberos Version 5 Specification 1 (5.1).

Encryption and checksum methods

The following encryption and checksum mechanisms must be supported. Implementations may support other mechanisms as well, but the additional mechanisms may only be used when communicating with principals known to also support them: Encryption: DES-CBC-MD5 Checksums: CRC-32, DES-MAC, DES-MAC-K, and DES-MD5

Realm Names

All implementations must understand hierarchical realms in both the Internet Domain and the X.500 style. When a ticket granting ticket for an unknown realm is requested, the KDC must be able to determine the names of the intermediate realms between the KDCs realm and the requested realm. 
Transited field encoding

DOMAIN-X500-COMPRESS (described in section 3.3.3.1) must be supported. Alternative encodings may be supported, but they may be used only when that encoding is supported by ALL intermediate realms.

Pre-authentication methods

The TGS-REQ method must be supported. The TGS-REQ method is not used on the initial request. The PA-ENC-TIMESTAMP method must be supported by clients but whether it is enabled by default may be determined on a realm by realm basis. If not used in the initial request and the error KDC_ERR_PREAUTH_REQUIRED is returned specifying PA-ENCTIMESTAMP as an acceptable method, the client should retry the initial request using the PA-ENC-TIMESTAMP preauthentication method. Servers need not support the PAENC-TIMESTAMP method, but if not supported the server should ignore the presence of PA-ENC-TIMESTAMP pre-authentication in a request.

Mutual authentication

Mutual authentication (via the KRB_AP_REP message) must be supported.

Ticket addresses and flags

All KDC's must pass on tickets that carry no addresses (i.e., if a TGT contains no addresses, the KDC will return derivative tickets), but each realm may set its own policy for issuing such tickets, and each application server will set its own policy with respect to accepting them. By default, servers should not accept them.

Proxies and forwarded tickets must be supported. Individual realms and application servers can set their own policy on when such tickets will be accepted.

All implementations must recognize renewable and postdated tickets, but need not actually implement them. If these options are not supported, the starttime and endtime in the ticket shall specify a ticket's entire useful life. When a postdated ticket is decoded by a server, all implementations shall make the presence of the postdated flag visible to the calling server.

User-to-user authentication

Support for user to user authentication (via the ENC-TKTIN-SKEY KDC option) must be provided by implementations, but individual realms may decide as a matter of policy to reject such requests on a perprincipal or realm-wide basis. 


\title{
Authorization data
}

Implementations must pass all authorization data subfields from ticket-granting tickets to any derivative tickets unless directed to suppress a subfield as part of the definition of that registered subfield type (it is never incorrect to pass on a subfield, and no registered subfield types presently specify suppression at the KDC) .

Implementations must make the contents of any authorization data subfields available to the server when a ticket is used.

Implementations are not required to allow clients to specify the contents of the authorization data fields.

\subsection{Recommended KDC values}

Following is a list of recommended values for a KDC implementation, based on the list of suggested configuration constants (see section $4.4)$.

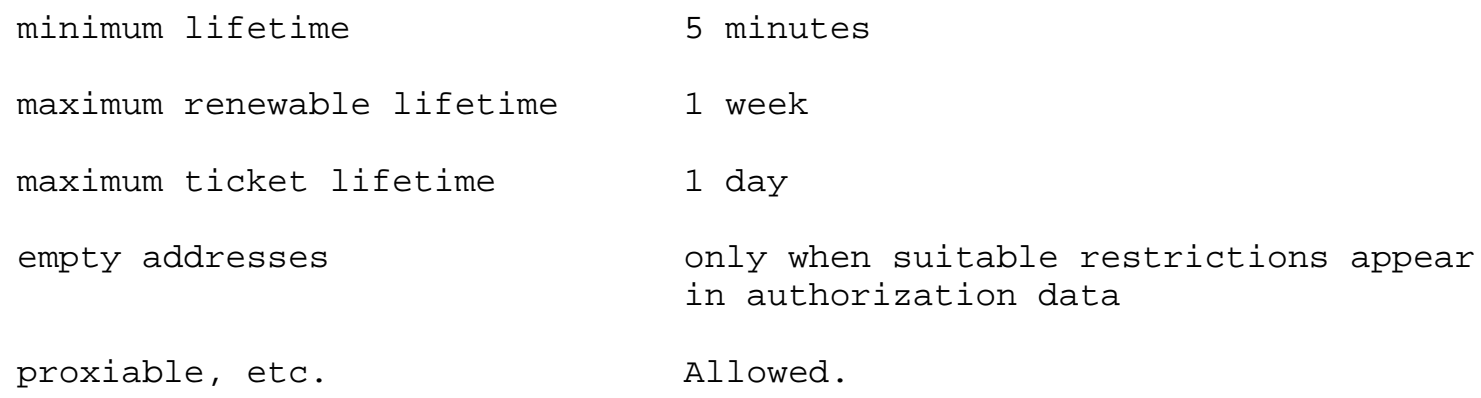

10. Acknowledgments

\begin{abstract}
Early versions of this document, describing version 4 of the protocol, were written by Jennifer Steiner (formerly at Project Athena); these drafts provided an excellent starting point for this current version 5 specification. Many people in the Internet community have contributed ideas and suggested protocol changes for version 5. Notable contributions came from Ted Anderson, Steve Bellovin and Michael Merritt [17], Daniel Bernstein, Mike Burrows, Donald Davis, Ravi Ganesan, Morrie Gasser, Virgil Gligor, Bill Griffeth, Mark Lillibridge, Mark Lomas, Steve Lunt, Piers McMahon, Joe Pato, William Sommerfeld, Stuart Stubblebine, Ralph Swick, Ted T'so, and Stanley Zanarotti. Many others commented and helped shape this specification into its current form.
\end{abstract}




\section{References}

[1] Miller, S., Neuman, C., Schiller, J., and J. Saltzer, "Section E.2.1: Kerberos Authentication and Authorization System", M.I.T. Project Athena, Cambridge, Massachusetts, December 21, 1987 .

[2] Steiner, J., Neuman, C., and J. Schiller, "Kerberos: An Authentication Service for Open Network Systems", pp. 191-202 in Usenix Conference Proceedings, Dallas, Texas, February, 1988.

[3] Needham, R., and M. Schroeder, "Using Encryption for Authentication in Large Networks of Computers", Communications of the ACM, Vol. 21 (12), pp. 993-999, December 1978.

[4] Denning, D., and G. Sacco, "Time stamps in Key Distribution Protocols", Communications of the ACM, Vol. 24 (8), pp. 533-536, August 1981 .

[5] Kohl, J., Neuman, C., and T. TS'O, "The Evolution of the Kerberos Authentication Service", in an IEEE Computer society Text soon to be published, June 1992 .

[6] Davis, D., and R. Swick, "Workstation Services and Kerberos Authentication at Project Athena", Technical Memorandum TM-424, MIT Laboratory for Computer Science, February 1990.

[7] Levine, P., Gretzinger, M, Diaz, J., Sommerfeld, W., and K. Raeburn, "Section E.1: Service Management System, M.I.T. Project Athena, Cambridge, Mas sachusetts (1987).

[8] CCITT, Recommendation X.509: The Directory Authentication Framework, December 1988 .

[9] Neuman, C., "Proxy-Based Authorization and Accounting for Distributed Systems," in Proceedings of the 13th International Conference on Distributed Computing Systems", Pittsburgh, PA, May 1993.

[10] Pato, J., "Using Pre-Authentication to Avoid Password Guessing Attacks", Open Software Foundation DCE Request for Comments 26, December 1992 .

[11] National Bureau of Standards, U.S. Department of Commerce, "Data Encryption Standard", Federal Information Processing Standards Publication 46, Washington, DC (1977). 
[12] National Bureau of Standards, U.S. Department of Commerce, "DES Modes of Operation", Federal Information Processing Standards Publication 81, Springfield, VA, December 1980.

[13] Stubblebine S., and V. Gligor, "On Message Integrity in Cryptographic Protocols", in Proceedings of the IEEE Symposium on Research in Security and Privacy, Oakland, California, May 1992 .

[14] International Organization for Standardization, "ISO Information Processing Systems - Data Communication High-Level Data Link Control Procedure - Frame Structure", IS 3309, October 1984, 3rd Edition.

[15] Rivest, R., "The MD4 Message Digest Algorithm", RFC 1320, MIT Laboratory for Computer Science, April 1992.

[16] Rivest, R., "The MD5 Message Digest Algorithm", RFC 1321, MIT Laboratory for Computer Science, April 1992.

[17] Bellovin S., and M. Merritt, "Limitations of the Kerberos Authentication System", Computer Communications Review, Vol. $20(5)$, pp. 119-132, October 1990 .

12. Security Considerations

Security issues are discussed throughout this memo.

13. Authors' Addresses

John Kohl

Digital Equipment Corporation

110 Spit Brook Road, M/S ZKO3-3/U14

Nashua, NH 03062

Phone: 603-881-2481

EMail: jtkohl@zk3.dec.com

B. Clifford Neuman USC/Information Sciences Institute 4676 Admiralty Way \#1001

Marina del Rey, CA 90292-6695

Phone: 310-822-1511

EMail: bcneisi.edu 
A. Pseudo-code for protocol processing

This appendix provides pseudo-code describing how the messages are to be constructed and interpreted by clients and servers.

A.1. KRB_AS_REQ generation

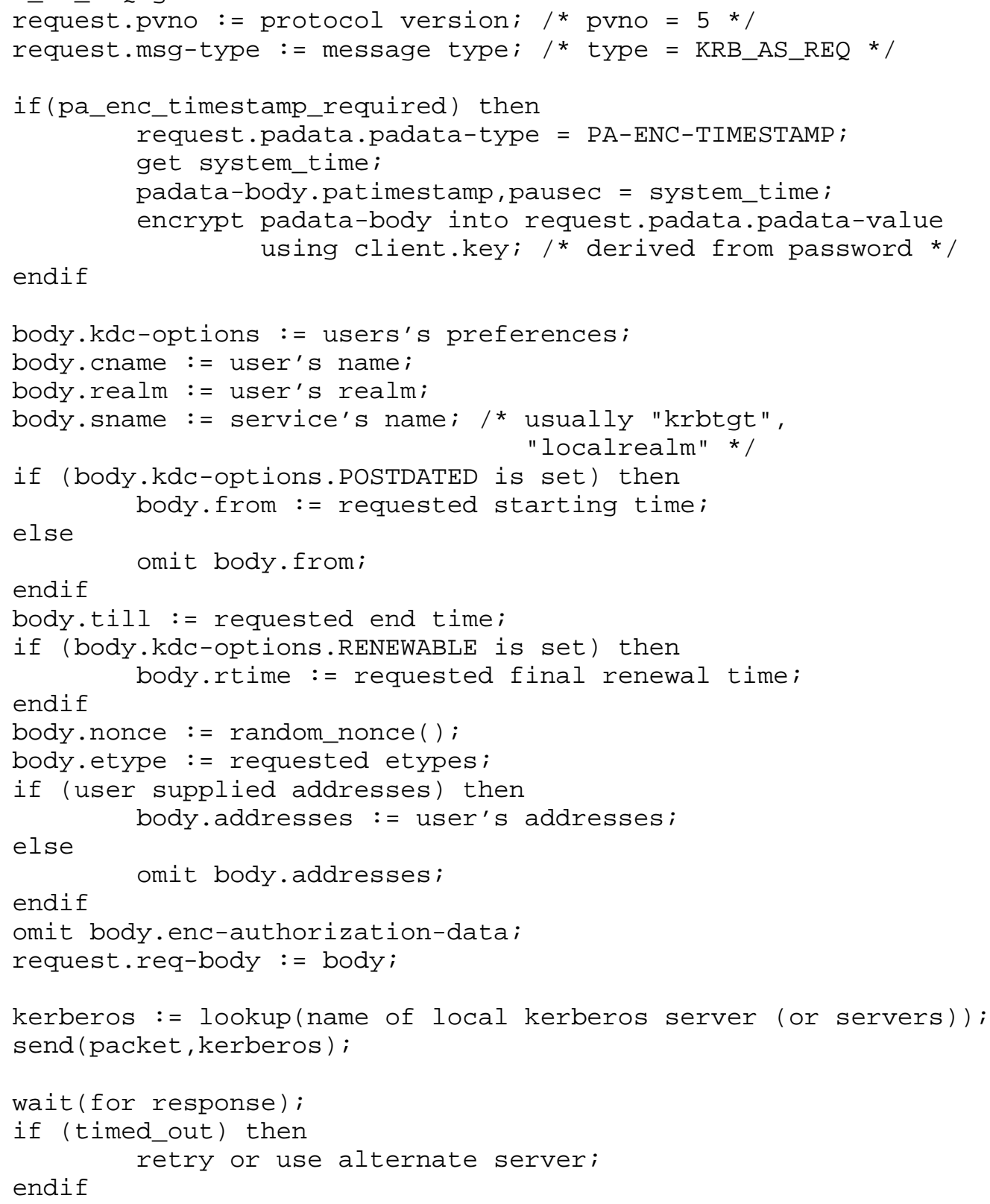


A.2. KRB_AS_REQ verification and KRB_AS_REP generation

decode message into req;

client := lookup (req. cname, req.realm);

server := lookup (req.sname, req.realm);

get system_time;

kdc_time := system_time.seconds;

if (!client) then

/* no client in Database */

error_out (KDC_ERR_C_PRINCIPAL_UNKNOWN) ;

endif

if (!server) then

/* no server in Database */

error_out (KDC_ERR_S_PRINCIPAL_UNKNOWN) ;

endif

if (client.pa_enc_timestamp_required and

pa_enc_timestamp not present) then

endif

error_out (KDC_ERR_PREAUTH_REQUIRED (PA_ENC_TIMESTAMP)) ;

if (pa_enc_timestamp present) then

decrypt req.padata-value into decrypted_enc_timestamp

using client.key;

using auth_hdr.authenticator.subkey;

if (decrypt_error()) then error_out (KRB_AP_ERR_BAD_INTEGRITY) ;

if (decrypted_enc_timestamp is not within allowable skew) then error_out (KDC_ERR_PREAUTH_FAILED);

endif

if (decrypted_enc_timestamp and usec is replay) error_out (KDC_ERR_PREAUTH_FAILED) ;

endif

endif

add decrypted_enc_timestamp and usec to replay cache;

use_etype := first supported etype in req.etypes;

if (no support for req.etypes) then

endif

error_out (KDC_ERR_ETYPE_NOSUPP) ;

new_tkt.vno:= ticket version; /* = 5*/

new_tkt.sname := req.sname;

new_tkt.srealm := req.srealm;

reset all flags in new_tkt.flags;

Kohl \& Neuman

[Page 92] 


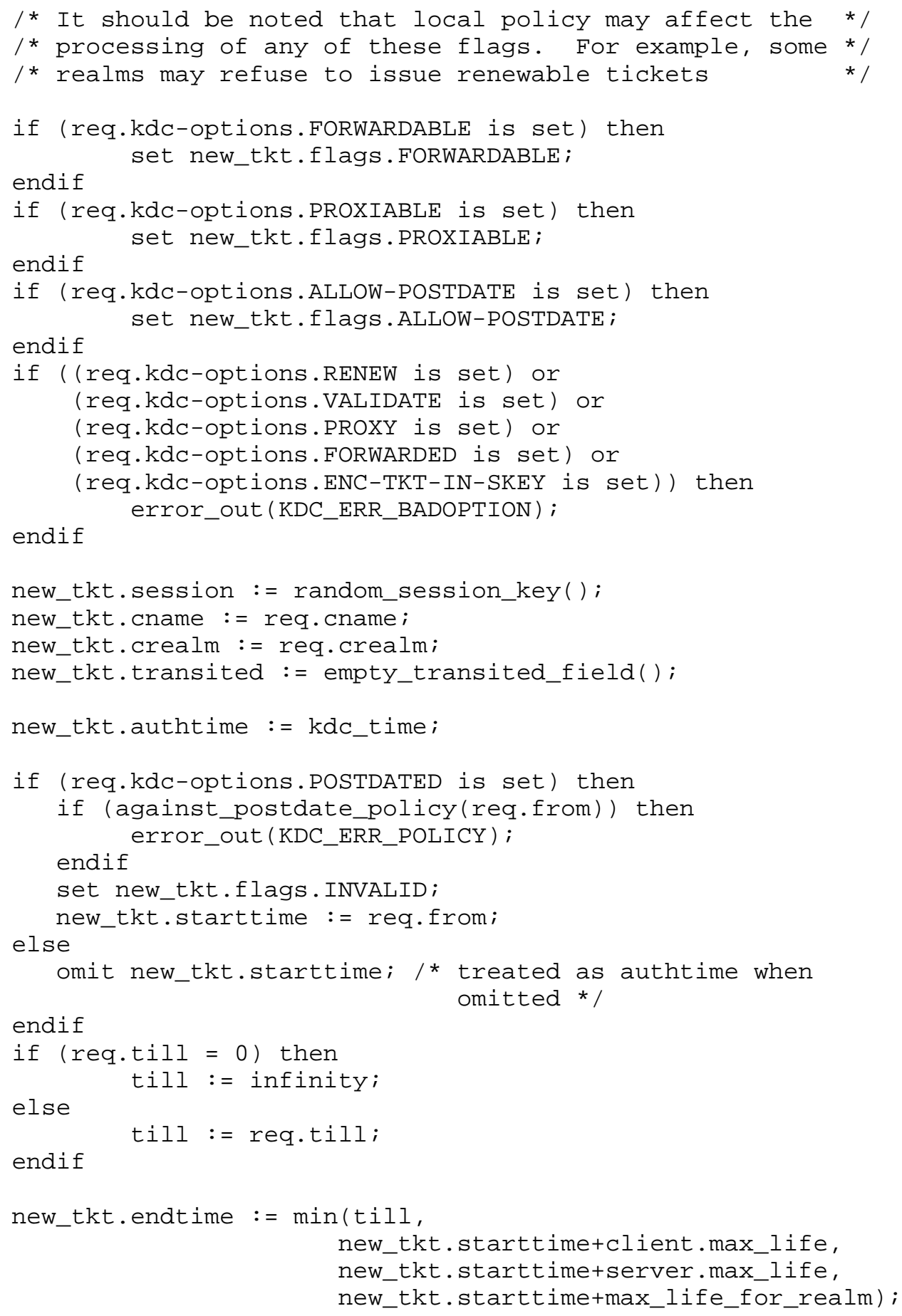




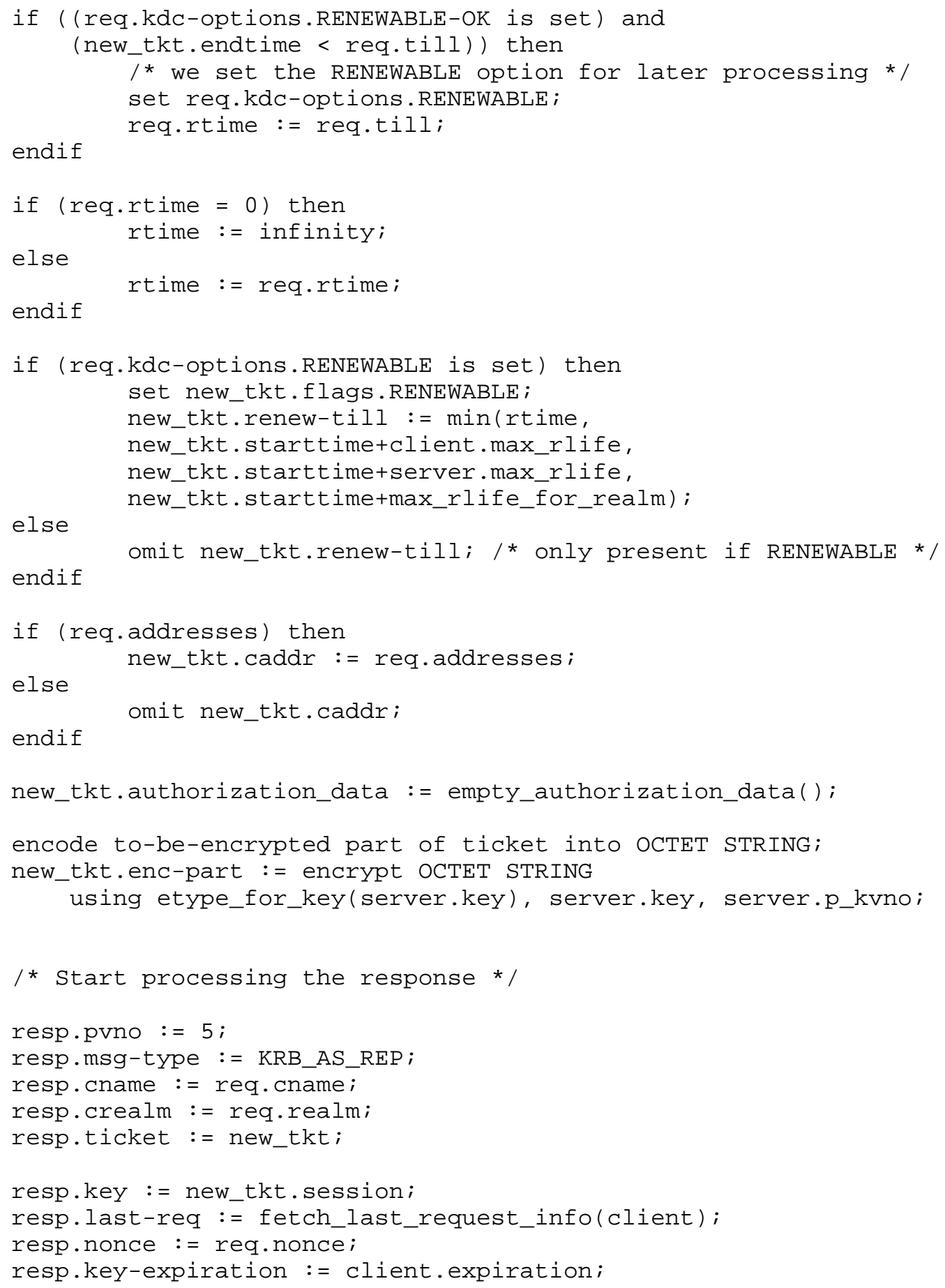




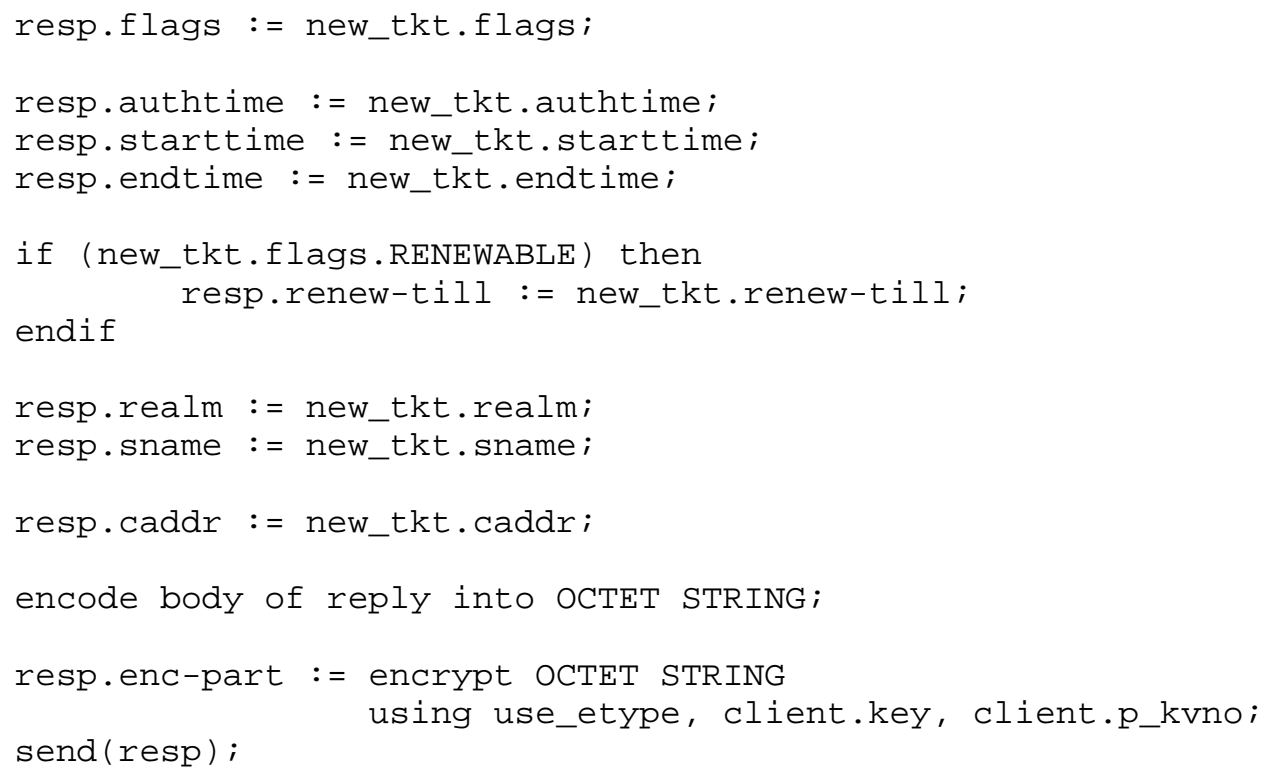


endif

print (warning message);

save_for_later(ticket, session, client, server,times, flags) ;

A.4. KRB_AS_REP and KRB_TGS_REP common checks

if (decryption_error() or

(req.cname $!=$ resp.cname) or

(req.realm != resp.crealm) or

(req.sname $!=$ resp.sname) or

(req.realm != resp.realm) or

(req.nonce $!=$ resp.nonce) or

(req.addresses $!=$ resp.caddr)) then

destroy resp.key;

endif

return KRB_AP_ERR_MODIFIED;

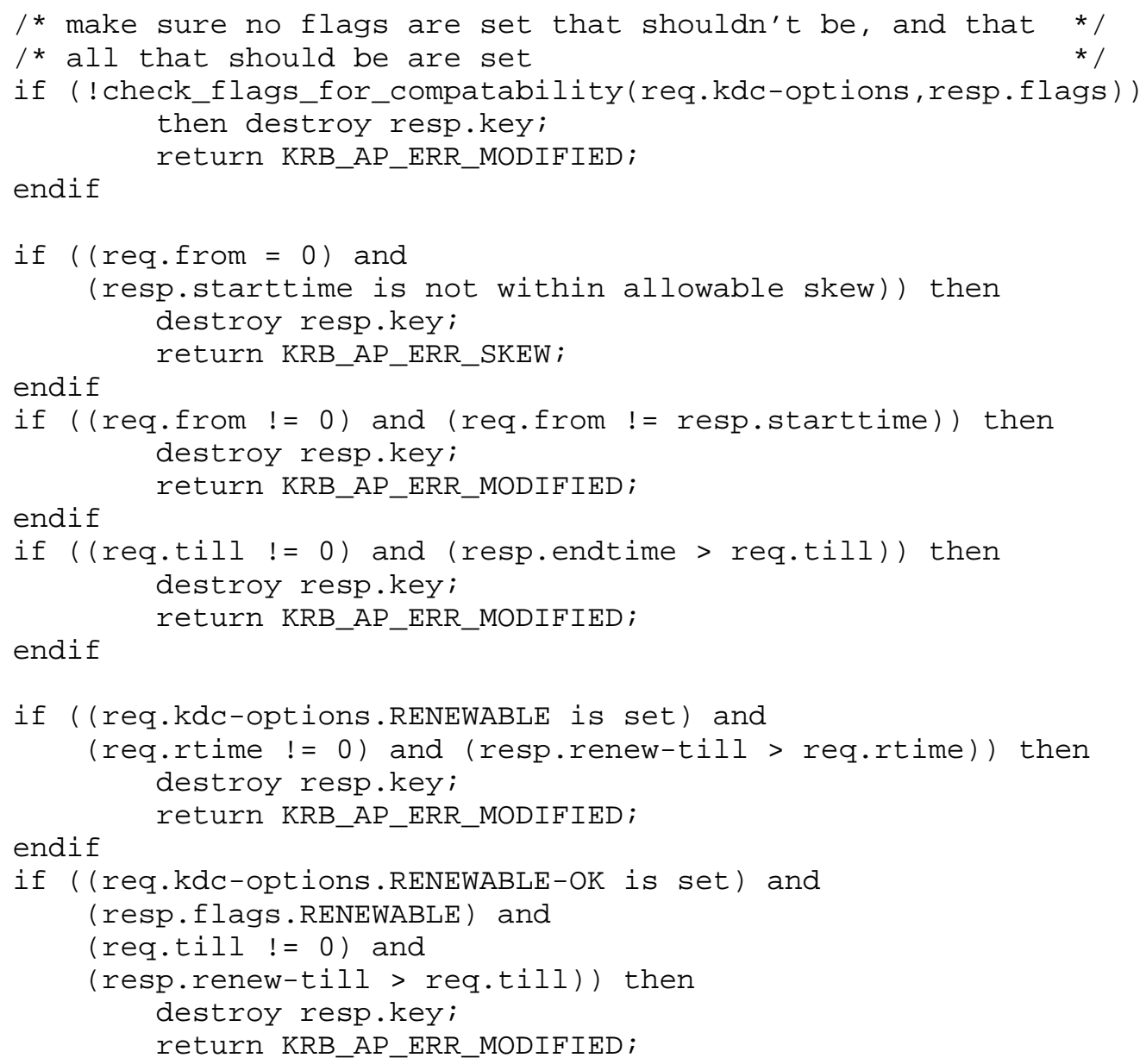

Kohl \& Neuman 
endif

A.5. KRB_TGS_REQ generation

$\begin{array}{ll}\text { /* Note that make_application_request might have to } & \text { */ } \\ \text { /* recursivly call this routine to get the appropriate } & \text { / } \\ \text { /* ticket-granting ticket } & \text { / }\end{array}$

request.pvno := protocol version; /* pvno $=5 * /$

request.msg-type $:=$ message type; /* type = KRB_TGS_REQ */

body.kdc-options := users's preferences;

/* If the TGT is not for the realm of the end-server */

/* then the sname will be for a TGT for the end-realm */

/* and the realm of the requested ticket (body.realm) */

/* will be that of the TGS to which the TGT we are */

/* sending applies

body.sname := service's name;

body.realm := service's realm;

if (body.kdc-options.POSTDATED is set) then

else body.from := requested starting time;

endif omit body.from;

body.till := requested end time;

if (body.kdc-options.RENEWABLE is set) then body.rtime := requested final renewal time;

endif

body.nonce := random_nonce ();

body.etype := requested etypes;

if (user supplied addresses) then

body.addresses := user's addresses;

else

endif

omit body.addresses;

body.enc-authorization-data := user-supplied data;

if (body.kdc-options.ENC-TKT-IN-SKEY) then

endif

body.additional-tickets_ticket := second $\mathrm{TGT}$;

request.req-body := body;

check := generate_checksum (req.body, checksumtype);

request.padata [0] padata-type := PA-TGS-REQ;

request.padata[0].padata-value := create a KRB_AP_REQ using

the TGT and checksum 


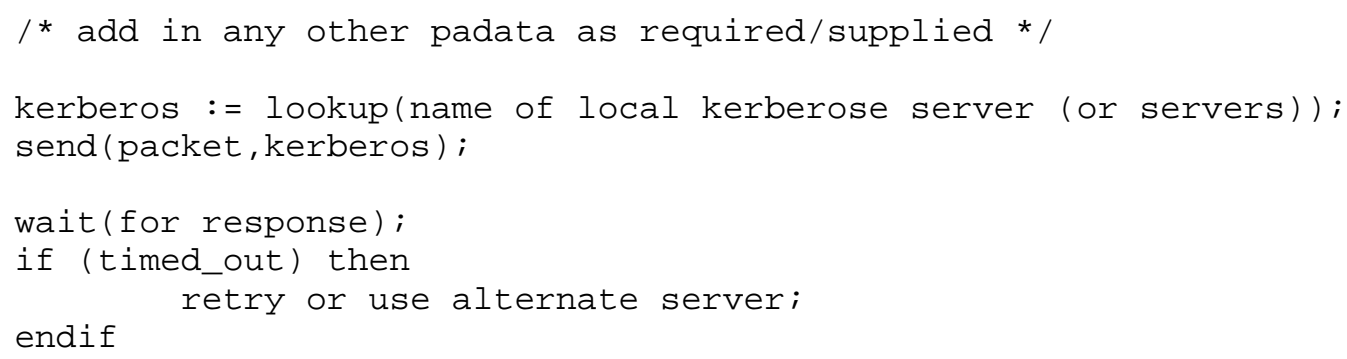

A.6. KRB_TGS_REQ verification and KRB_TGS_REP generation /* note that reading the application request requires first determining the server for which a ticket was issued, and choosing the correct key for decryption. The name of the server appears in the plaintext part of the ticket. */ 


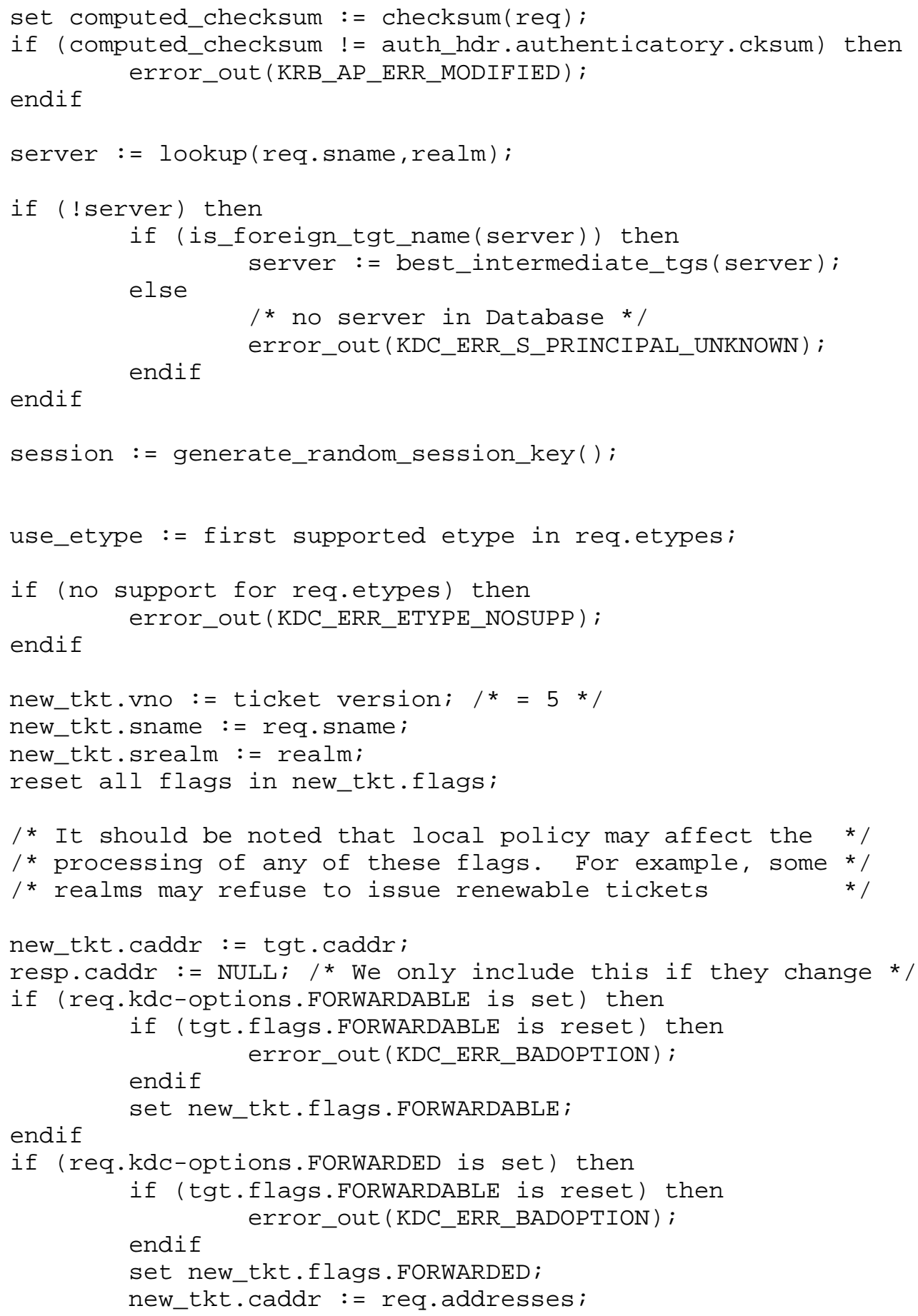




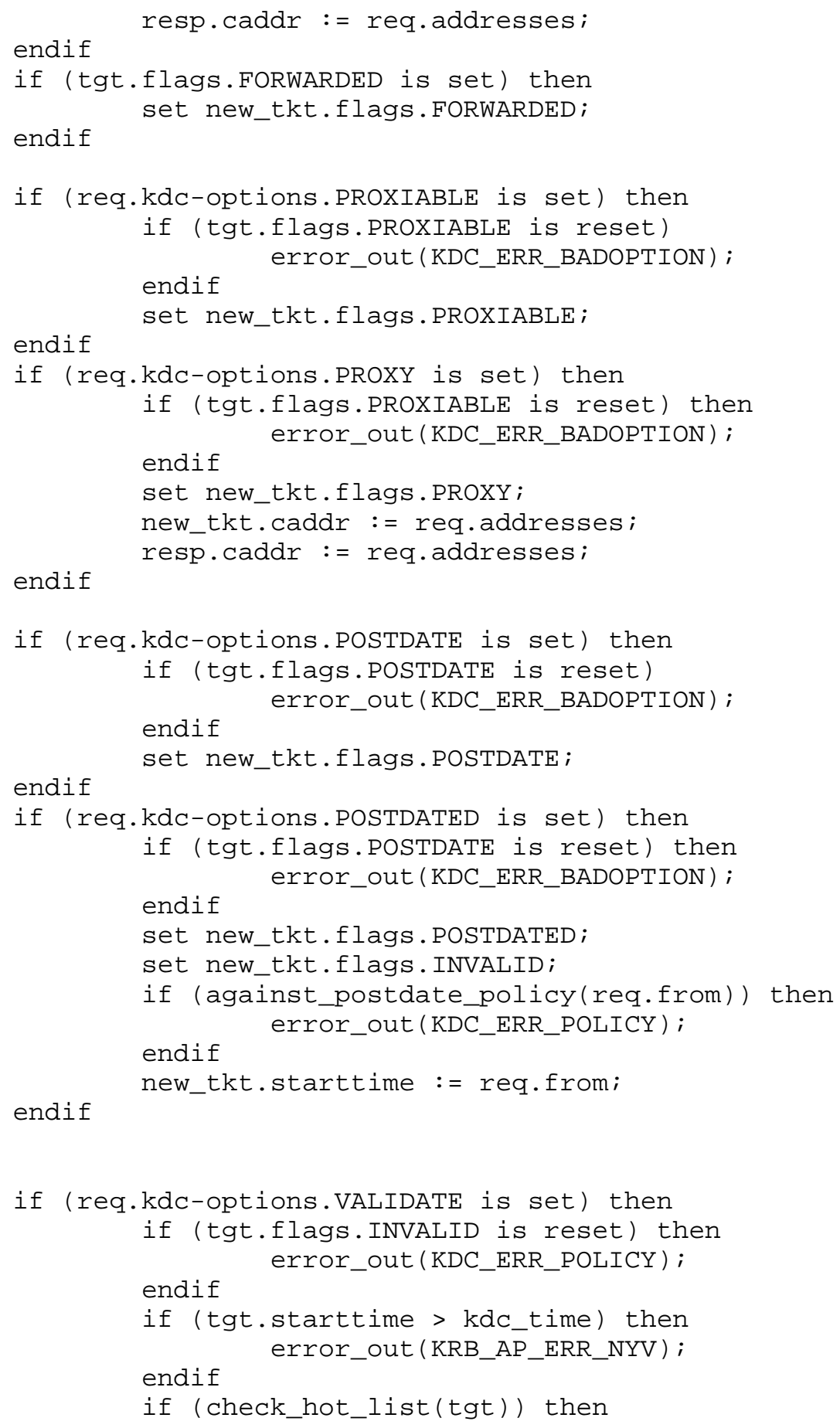




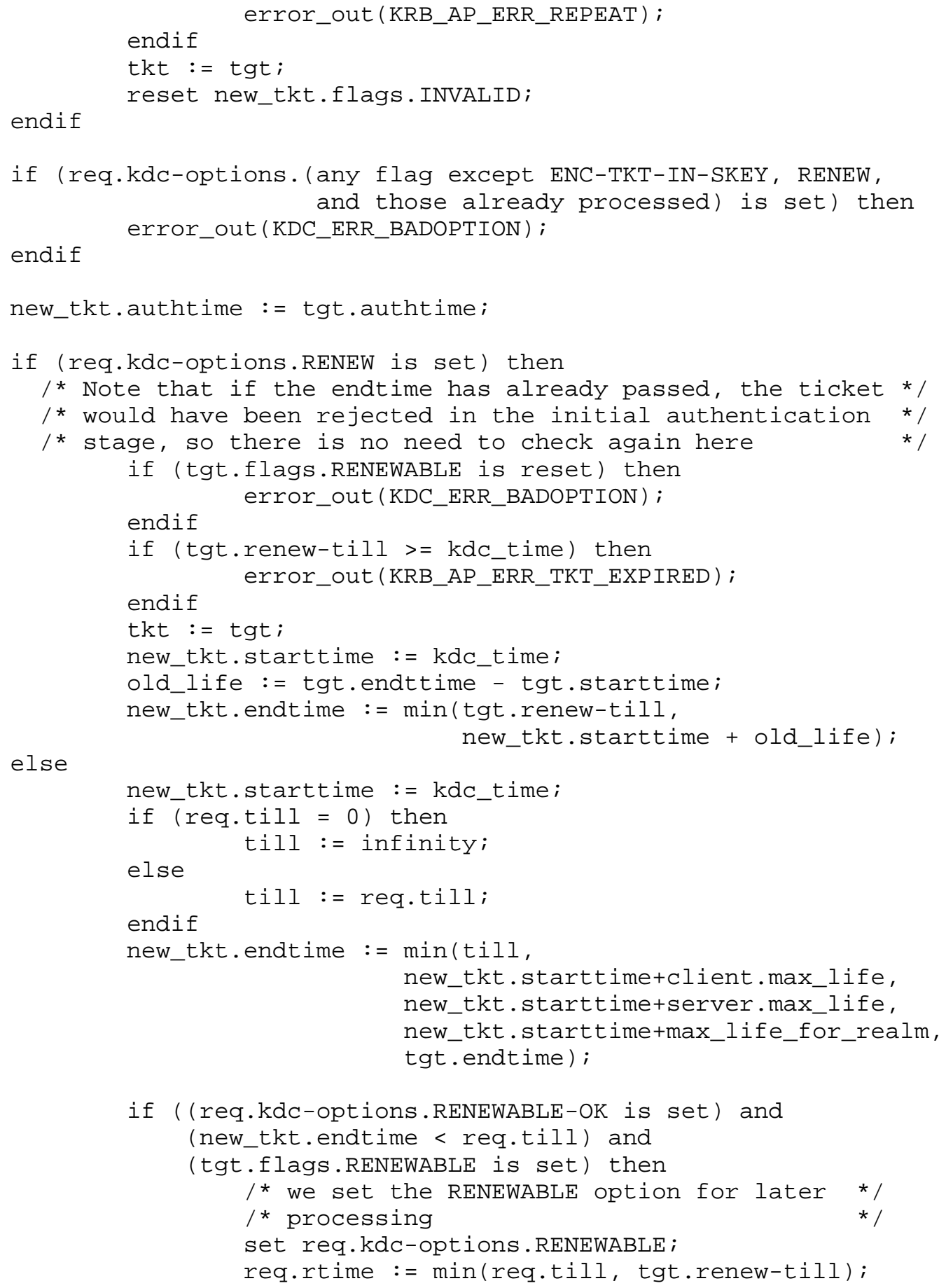

Kohl \& Neuman 


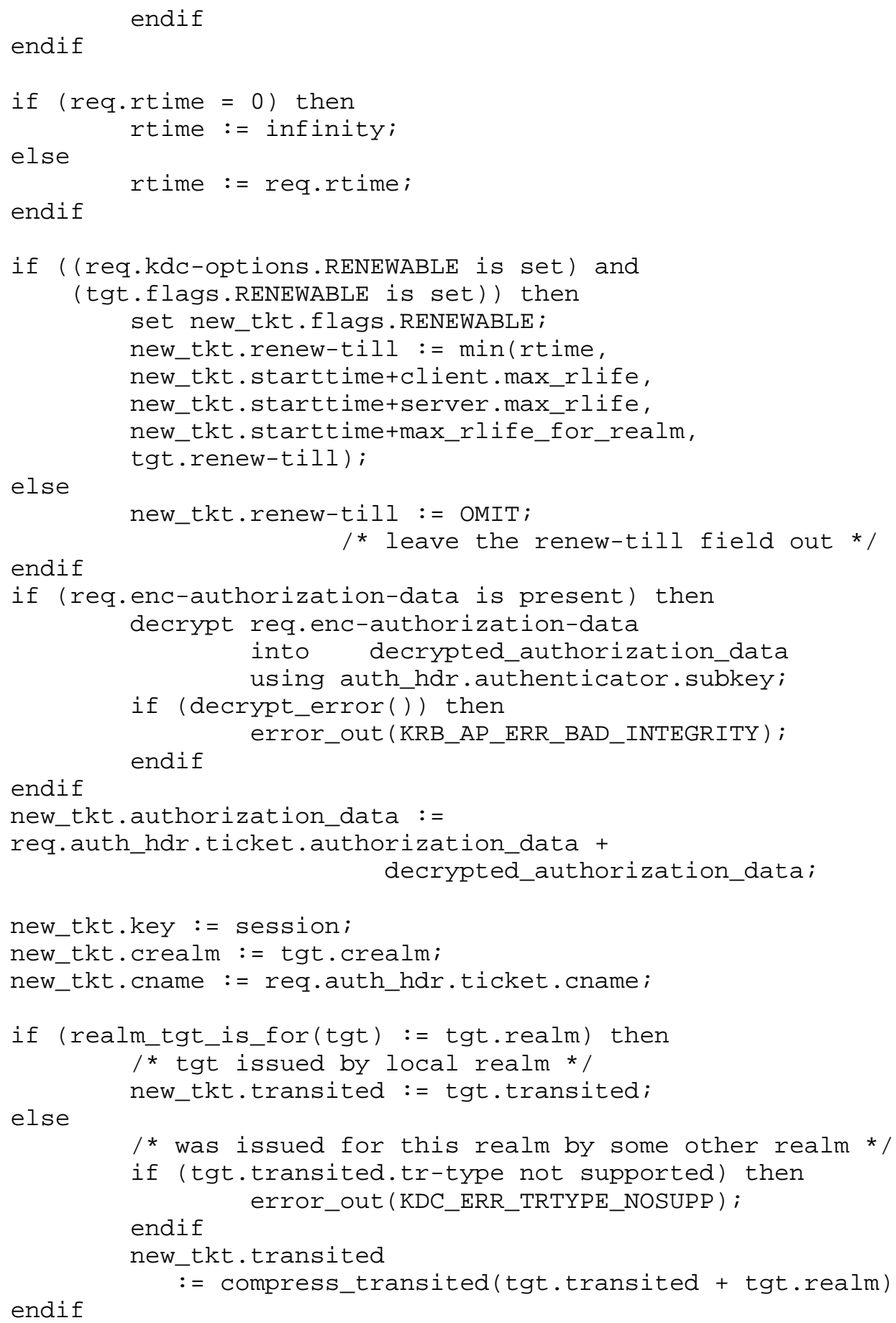




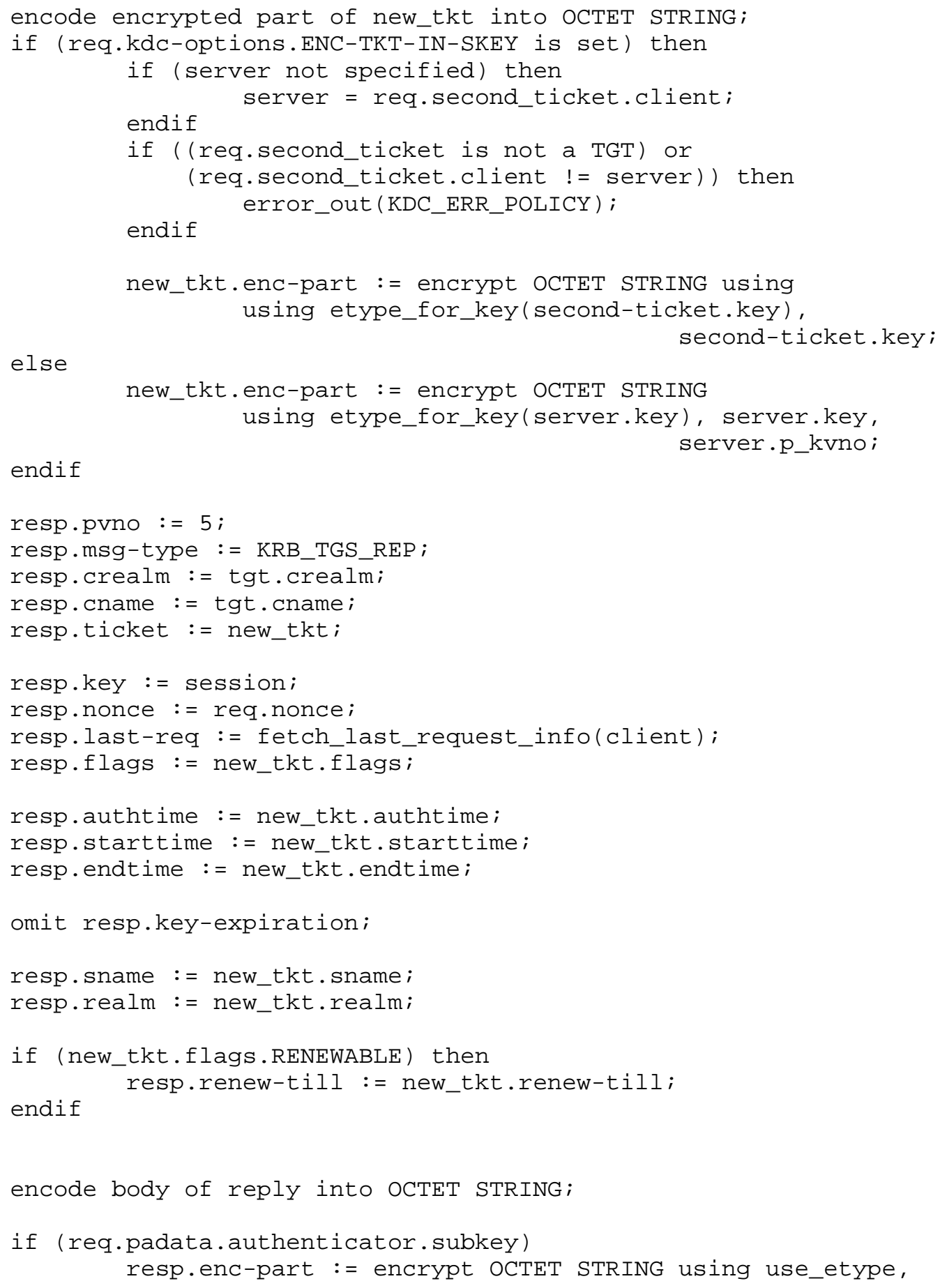




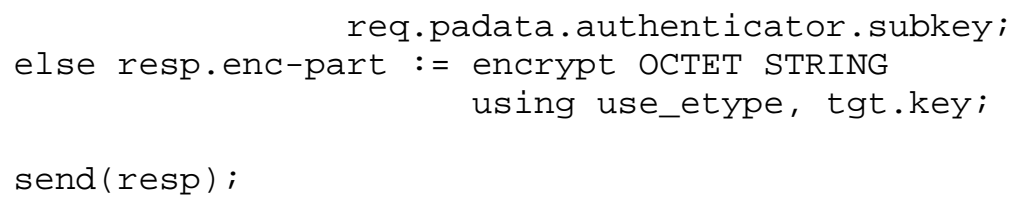

A.8. Authenticator generation

body.authenticator-vno := authenticator vno; / = 5 */

body.cname, body.crealm:= client name;

if (supplying checksum) then body.cksum := checksum;

endif

get system_time;

body.ctime, body.cusec := system_time;

if (selecting sub-session key) then select sub-session key; 
A.9. KRB_AP_REQ generation

obtain ticket and session_key from cache;

packet.pvno := protocol version; /* 5 */

packet.msg-type := message type; /* KRB_AP_REQ */

if (desired(MUTUAL_AUTHENTICATION)) then

set packet.ap-options.MUTUAL-REQUIRED;

else

endif

reset packet.ap-options.MUTUAL-REQUIRED;

if (using session key for ticket) then

set packet.ap-options.USE-SESSION-KEY;

else

endif

packet.ticket := ticket; /* ticket */

generate authenticator;

encode authenticator into OCTET STRING;

encrypt OCTET STRING into packet.authenticator using session_key;

A.10. KRB_AP_REQ verification

receive packet;

if (packet.pvno !=5) then

either process using other protocol spec

or error_out (KRB_AP_ERR_BADVERSION);

endif

if (packet.msg-type != KRB_AP_REQ) then

error_out (KRB_AP_ERR_MSG_TYPE) ;

endif

if (packet.ticket.tkt_vno !=5) then

either process using other protocol spec

or error_out (KRB_AP_ERR_BADVERSION);

endif

if (packet.ap_options.USE-SESSION-KEY is set) then retrieve session key from ticket-granting ticket for packet.ticket.\{sname, srealm,enc-part.etype\};

else

retrieve service key for

packet.ticket.\{sname, srealm, enc-part.etype, enc-part.skvno\}; endif

if (no_key_available) then

if (cannot_find_specified_skvno) then

else

error_out (KRB_AP_ERR_BADKEYVER);

endif

error_out (KRB_AP_ERR_NOKEY);

Kohl \& Neuman

[Page 105] 


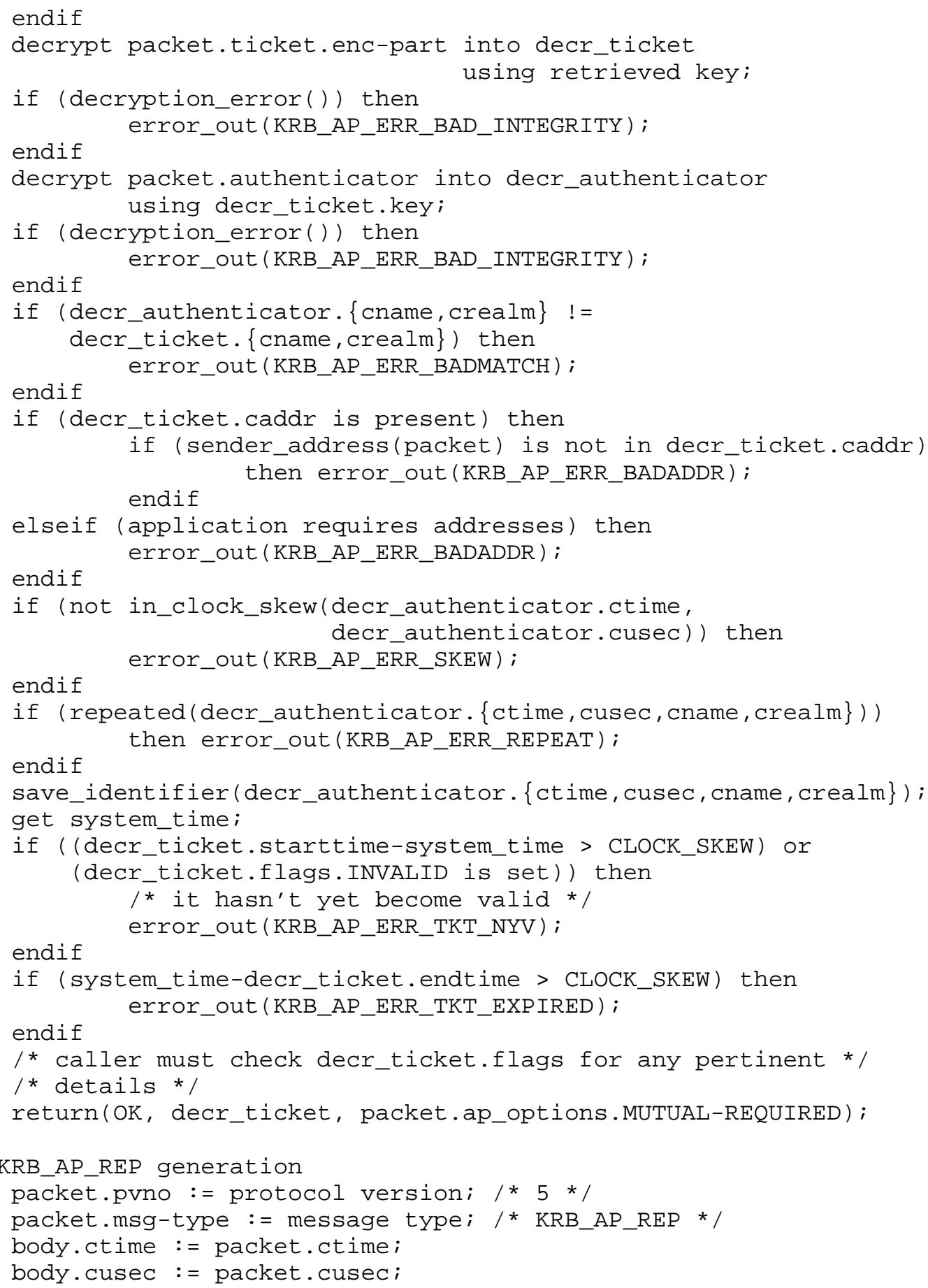




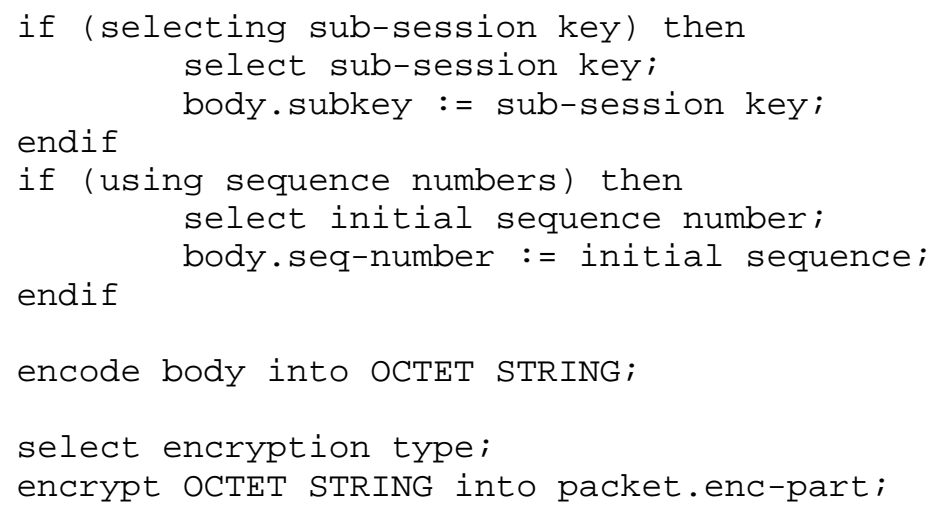

A.13. KRB_SAFE generation

collect user data in buffer;

/* assemble packet: */ 


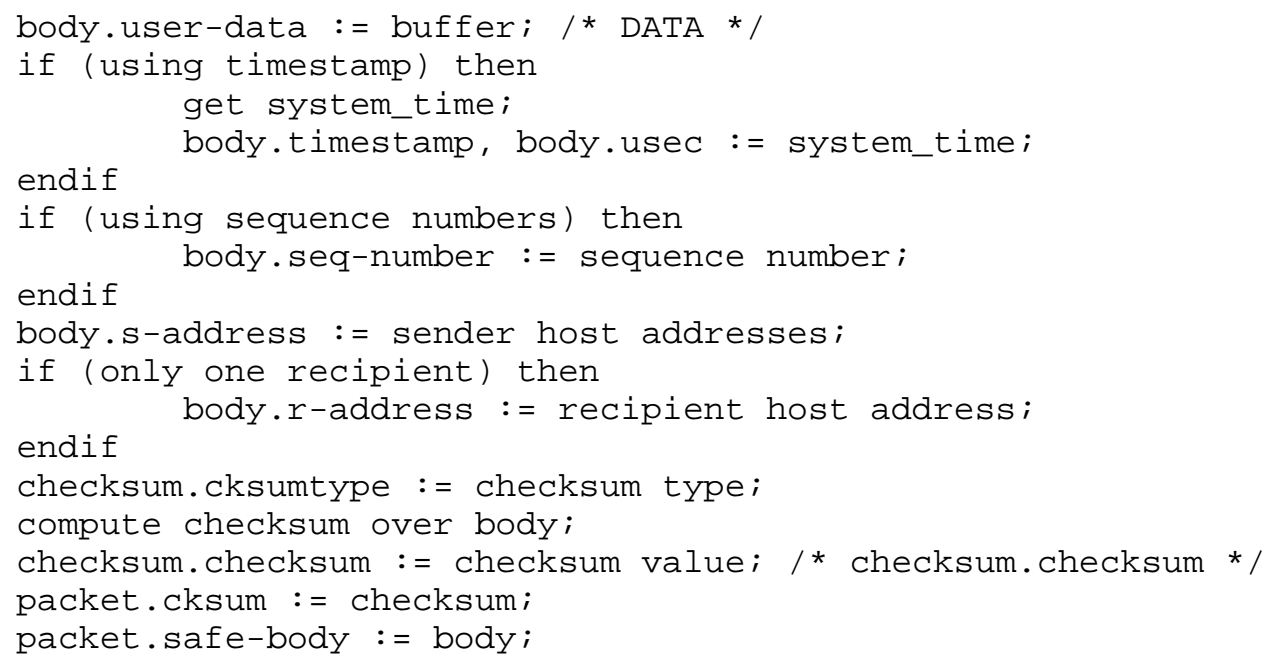

A.14. KRB_SAFE verification

A.15. KRB_SAFE and KRB_PRIV common checks

if (packet.s-address $!=0 /$ __sender (packet)) then

/* O/S report of sender not who claims to have sent it */ endif 


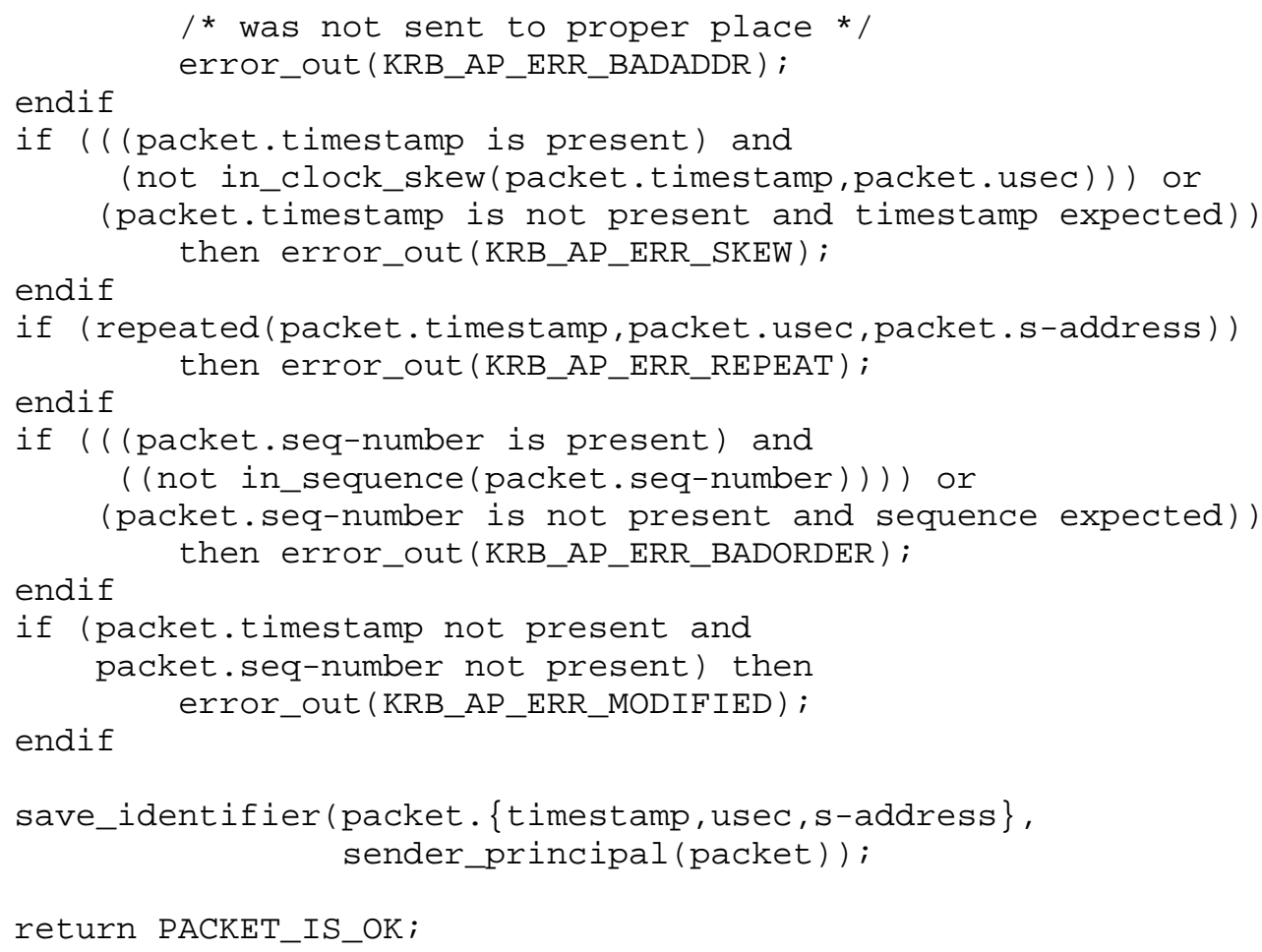


encode body into OCTET STRING;

select encryption type;

encrypt OCTET STRING into packet.enc-part.cipher;

A.17. KRB_PRIV verification

receive packet;

if (packet.pvno $!=5$ ) then

either process using other protocol spec

or error_out (KRB_AP_ERR_BADVERSION);

endif

if (packet.msg-type $!=$ KRB_PRIV) then

error_out (KRB_AP_ERR_MSG_TYPE) ;

endif

cleartext := decrypt (packet.enc-part) using negotiated key;

if (decryption_error()) then

endif

error_out (KRB_AP_ERR_BAD_INTEGRITY) ;

if (safe_priv_common_checks_ok(cleartext)) then

return(cleartext.DATA, PACKET_IS_GENUINE_AND_UNMODIFIED) ; else

endif

return common_checks_error;

A.18. KRB_CRED generation

invoke KRB_TGS; /* obtain tickets to be provided to peer */

/* assemble packet: */

packet.pvno := protocol version; /* $5 * /$

packet.msg-type := message type; /* KRB_CRED */

for (tickets[n] in tickets to be forwarded) do packet.tickets $[n]=$ tickets $[n]$.ticket;

done

packet.enc-part.etype := encryption type;

for (ticket $[n]$ in tickets to be forwarded) do body.ticket-info[n].key = tickets[n].session; body.ticket-info[n].prealm = tickets[n].crealm;

body.ticket-info[n].pname = tickets[n]. cname; body.ticket-info[n].flags = tickets[n].flags; body.ticket-info[n].authtime = tickets[n].authtime; body.ticket-info[n].starttime = tickets[n].starttime; body.ticket-info[n].endtime = tickets $[n]$.endtime;

body.ticket-info[n].renew-till = tickets[n].renew-till; 


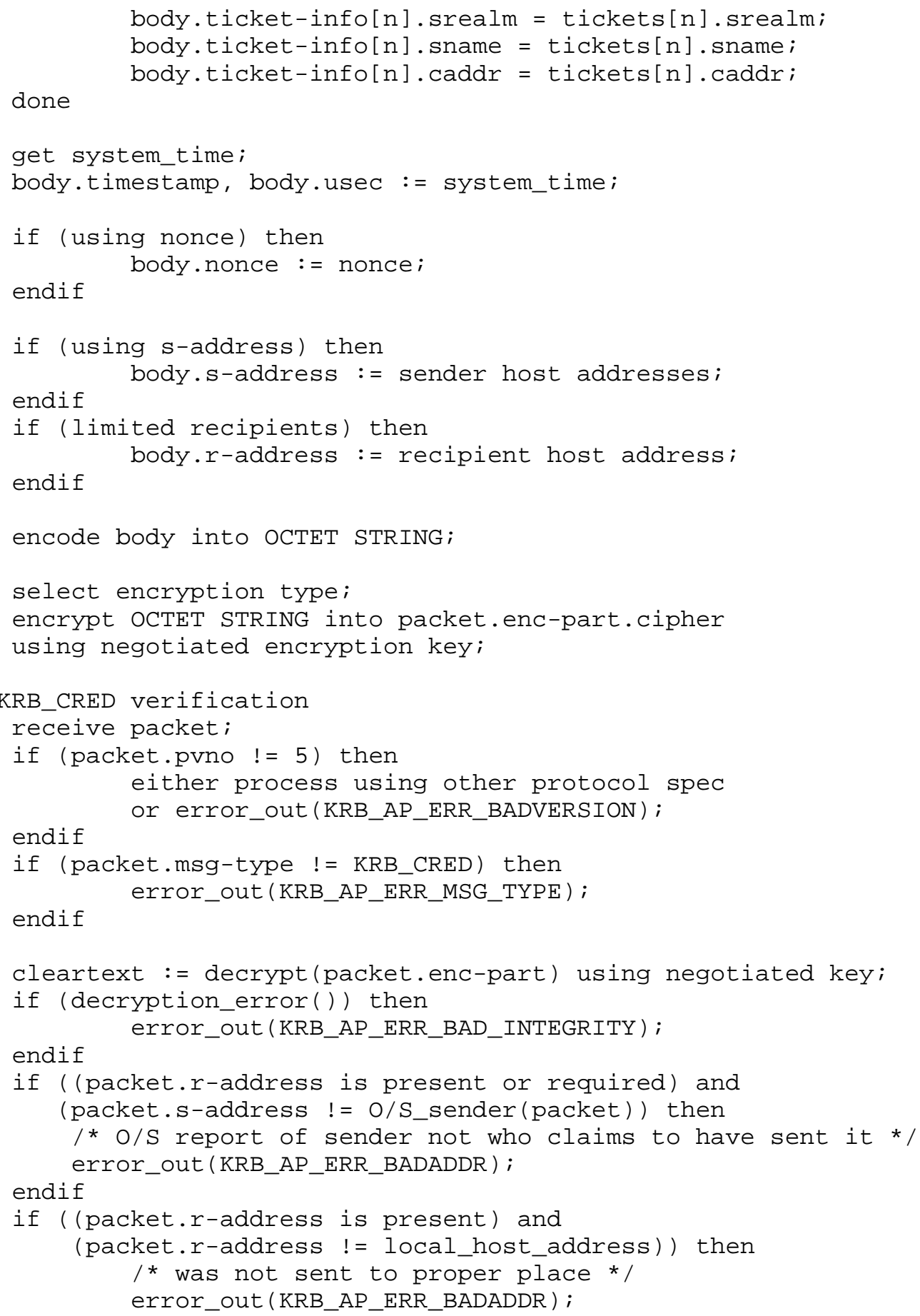

Kohl \& Neuman 


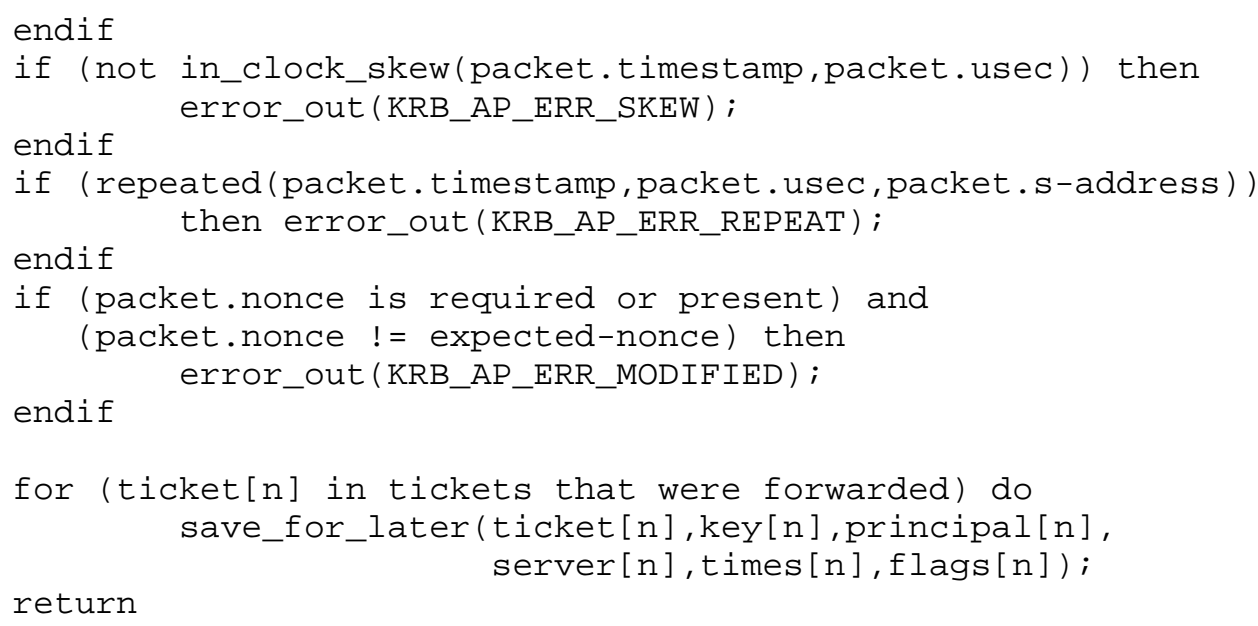

A.20. KRB_ERROR generation

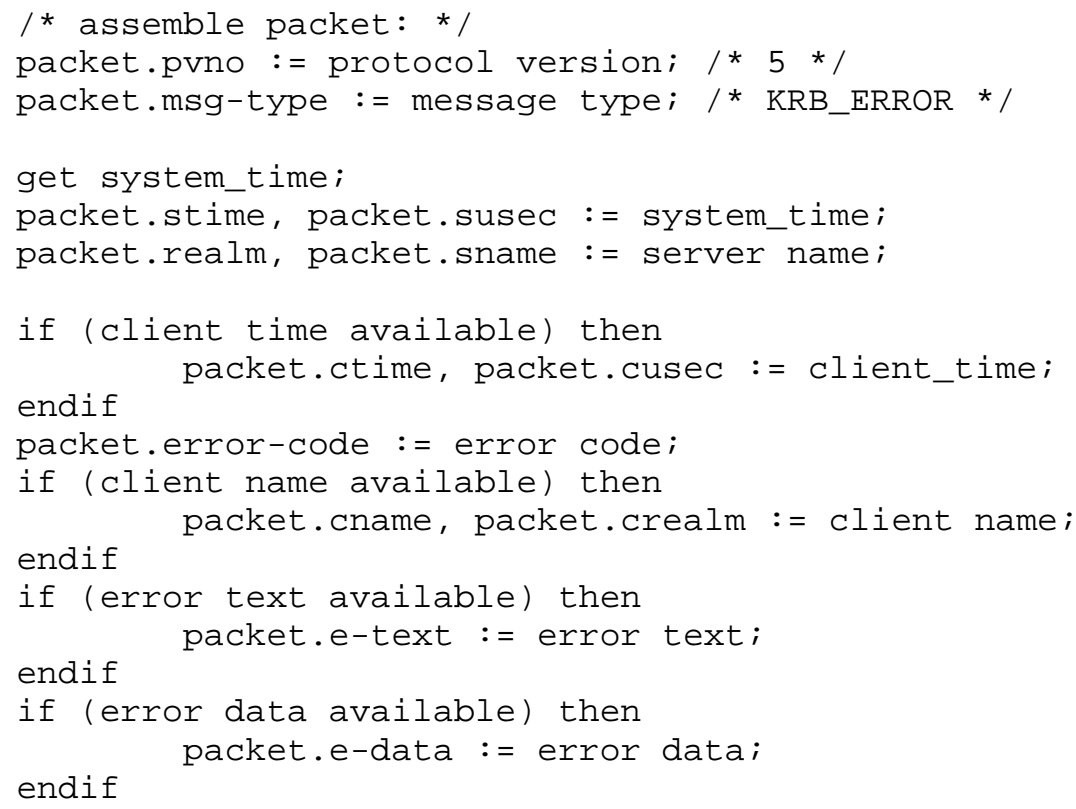

